\title{
QUANTUM LANGLANDS DUALITIES OF BOUNDARY CONDITIONS, $D$-MODULES, AND CONFORMAL BLOCKS
}

\author{
EDWARD FRENKEL AND DAVIDE GAIOTTO
}

\begin{abstract}
We review and extend the vertex algebra framework linking gauge theory constructions and a quantum deformation of the Geometric Langlands Program. The relevant vertex algebras are associated to junctions of two boundary conditions in a $4 \mathrm{~d}$ gauge theory and can be constructed from the basic ones by following certain standard procedures. Conformal blocks of modules over these vertex algebras give rise to twisted $D$ modules on the moduli stacks of $G$-bundles on Riemann surfaces which have applications to the Langlands Program. In particular, we construct a series of vertex algebras for every simple Lie group $G$ which we expect to yield $D$-module kernels of various quantum Geometric Langlands dualities. We pay particular attention to the full duality group of gauge theory, which enables us to extend the standard qGL duality to a larger duality groupoid. We also discuss various subtleties related to the spin and gerbe structures and present a detailed analysis for the $U(1)$ and $S U(2)$ gauge theories.
\end{abstract}

\section{Contents}

1. Introduction

2. Overview of the gauge theory setup

3. Boundary conditions, dualities, and junction vertex algebras

4. Quantum Langlands dualities of twisted $D$-modules 29

5. A family of kernel vertex algebras 35

6. Branes, twisted $D$-modules, and compactification functors 40

7. A simple example: $U(1)$ gauge theory 56

8. A richer example: $S U(2)$ gauge theory 64

9. General gauge groups and discrete $\theta$ angles 75

10. Other duality kernels 79

11. Some future directions 84

References 85

\section{INTRODUCTION}

A geometric version of the Langlands Program originated in the 1980s in the works of Deligne, Drinfeld, and Laumon. In the early 1990s, Beilinson and Drinfeld discovered its deep connections to $2 \mathrm{~d}$ conformal field theory and representation theory of affine KacMoody algebras at the critical level [11]. This led them to the insight that deforming the Kac-Moody level, one should be able to obtain a quantum deformation of the Geometric 
Langlands correspondence (see 65] for an early formulation). Today, this is usually stated as a conjectural equivalence between derived categories of twisted $D$-modules on the moduli stacks $\operatorname{Bun}_{G}$ and $\operatorname{Bun}_{L_{G}}$ of bundles on a compact Riemann surface $X$ for a pair of Langlands dual simple ${ }^{1}$ Lie groups $G$ and ${ }^{L} G$ :

$$
S_{m}: \quad D_{\kappa}\left(\operatorname{Bun}_{G}\right) \longleftrightarrow D_{-1 / m \kappa}\left(\operatorname{Bun}_{L_{G}}\right)
$$

where $m$ is the lacing number of $G$ and we equip $X$ with a spin structure $\varrho^{2}$ (it is expected that $S_{m}^{2}$ is the identity in the categorical sense). Here $\kappa$ is a twisting parameter for $D$-modules corresponding to the level of the affine Kac-Moody algebra $\widehat{\mathfrak{g}}$ shifted by the critical value. The equivalence $(1.1)$ is expected to hold for irrational $\kappa$ (i.e. $\kappa \in \mathbb{C} \backslash \mathbb{Q}$ ) and satisfy some natural compatibilities. For rational $\kappa$ some modifications may have to be applied to the above categories for it to hold.

The ordinary (non-quantum) Geometric Langlands correspondence appears in the $\kappa \rightarrow 0$ limit of (1.1). In this limit $D_{-1 / m \kappa}\left(\operatorname{Bun}_{L_{G}}\right)$ becomes a suitably modified version of the derived category of quasicoherent sheaves on $\operatorname{Loc}_{L_{G}}$, the moduli stack of flat ${ }^{L} G$-bundles on $X$ (see [6] for a precise conjecture).

In the past twenty years, a great effort has been made to search for general methods and structures that could shed light on how and why quantum Geometric Langlands (qGL) duality (1.1) comes about (see [23, [55, 54, 67, 44, 46, 36, 37, 16, 1] for a partial list of references).

It turns out that a lot of valuable information can be obtained from the study of dualities in $4 \mathrm{~d}$ supersymmetric gauge theories. In their groundbreaking work [55], Kapustin and Witten related quantum qGL duality (1.1) to the $S$-duality of twisted 4 d gauge theories and mirror symmetry of Hitchin moduli spaces (see also [54, 68]). In their approach, $S$-duality is manifested as an equivalence of certain categories of branes on the Hitchin moduli spaces for $G$ and ${ }^{L} G$, which they have linked to categories of $D$-modules. On the other hand, Witten and one of the authors undertook in [40, 41] an in-depth study of boundary conditions in $4 \mathrm{~d}$ gauge theory and their behavior under quantum dualities. This dramatically expanded the class of $4 \mathrm{~d}$ gauge theory data that one could employ to gain further insights into the qGL dualities.

In this paper, we build on these results, as well as the recent works [36, 37, 39, 16, to present a systematic study of the quantum Geometric Langlands dualities in the framework of boundary conditions in $4 \mathrm{~d}$ gauge theory and the corresponding junction vertex algebras. Here we list the main ingredients of our approach:

(i) We heed a lesson of $4 \mathrm{~d}$ gauge theory and consider, instead of a single duality $S_{m}$ sending $\kappa \mapsto-1 / m \kappa$ and $G \mapsto{ }^{L} G$, the full group (more precisely, a groupoid) of quantum dualities. It combines $S_{m}$ with the dualities $T^{n}$ sending $\kappa \rightarrow \kappa+n, n \in$ $n(G) \cdot \mathbb{Z}$ and $G \mapsto G$, which correspond to the equivalences

$$
D_{\kappa}\left(\operatorname{Bun}_{G}\right) \longleftrightarrow D_{\kappa+n}\left(\operatorname{Bun}_{G}\right)
$$

\footnotetext{
${ }^{1}$ This can be generalized to reductive Lie groups. For abelian $G$, it has been proved in 60.

${ }^{2}$ Without a choice of spin structure the statement is modified in an interesting way, which we will review later on.
} 
given by taking the tensor product with a power of a "minimal" line bundle $\mathcal{L}_{G}$ on $\operatorname{Bun}_{G}$ (corresponding to level $n(G)$ defined in Section 3.3). The functors $S_{m}$ and $T^{n}$ are then expected to generate a categorical action of a certain groupoid $\mathcal{G}_{\kappa}^{G}$ on the categories of twisted $D$-modules on $\operatorname{Bun}_{G}$ (at least, for irrational $\kappa$ ). Thus, for any $g \in \mathcal{G}_{\kappa}^{G}$ we should have functors

$$
\mathcal{E}_{\kappa}^{G, g}: D_{\kappa}\left(\operatorname{Bun}_{G}\right) \rightarrow D_{g(\kappa)}\left(\operatorname{Bun}_{g(G)}\right),
$$

satisfying the relations in the groupoid $\mathcal{G}_{\kappa}^{G}$, which ultimately boil down to relations between the elements

$$
S_{m}=\left(\begin{array}{cc}
0 & -1 \\
m & 0
\end{array}\right) \quad T=\left(\begin{array}{ll}
1 & 1 \\
0 & 1
\end{array}\right)
$$

in the group $P G L_{2}(\mathbb{Z})$. The study of all of these functors together (rather than $S_{m}$ of (1.1) alone) allows for a much greater flexibility and leads to a number of non-trivial consequences $3^{3}$

(ii) We start with the basic boundary conditions of the twisted $4 \mathrm{~d}$ gauge theory labeled by $(G, \kappa)$ : Dirichlet, Neumann, and Nahm, and consider their images under the action of the groupoid $\mathcal{G}_{\kappa}^{G}$. Thus, we obtain a big collection of boundary conditions $B$. To each of them corresponds a ribbon category $\mathcal{C}_{\kappa}^{G}(B)$ of line defects (or a spin-ribbon category if we choose a spin structure on the underlying manifold; we could also view them as chiral categories). In general, this is a derived, or a DG, category. We describe these categories for the basic boundary conditions explicitly (see Section 3.1). We then assign to a boundary condition $g(B)$ with label $(g(G), g(\kappa))$, where $B$ is basic and $g \in \mathcal{G}_{\kappa}^{G}$, the same category $\mathcal{C}_{\kappa}^{G}(B)$. All equivalences of categories that this assignment entails are assumed to hold (in particular, this way we naturally get the statement that $\mathcal{C}_{\kappa}^{G}$ (Neumann) is equivalent to $\mathcal{C}_{1 / m \kappa}^{L}(\mathrm{Nahm})$, which is related to a conjecture of Gaitsgory and Lurie [44, 46]).

(iii) For each Riemann surface $X$ and a point $x \in X$, there is a compactification functor from the category of line defects $\mathcal{C}_{\kappa}^{G}(B)$ to $D_{\kappa}\left(\operatorname{Bun}_{G}\right)$. Thus, we can go from line defects of boundary conditions directly to objects of the derived category $D_{\kappa}\left(\operatorname{Bun}_{G}\right)$ (in what follows we will refer to them simply as twisted $D$-modules on $\operatorname{Bun}_{G}$ ), bypassing the categories of branes. These functors have multi-point generalizations.

(iv) To each junction of boundary conditions $B_{1} \rightarrow B_{2}$ in the $4 \mathrm{~d}$ theory labeled by $(G, \kappa)$ one associates a vertex algebra $V_{\kappa}^{G}\left(B_{1} \rightarrow B_{2}\right)$ and a functor

$$
\mathcal{C}_{\kappa}^{G}\left(B_{1}\right)^{\vee} \otimes \mathcal{C}_{\kappa}^{G}\left(B_{2}\right) \rightarrow V_{\kappa}^{G}\left(B_{1} \rightarrow B_{2}\right)-\bmod .
$$

\footnotetext{
${ }^{3}$ For a non-simply connected group $G$ only powers of $T$ divisible by a positive integer $n(G)$ are allowed as quantum dualities. However, gauge theory predicts that an extension of the duality groupoid $\mathcal{G}_{\kappa}^{G}$, generated by $S_{m}$ and $T$, acts on the more general categories of gerbe-twisted $D$-modules on Bun $G^{\prime}$ where $G^{\prime}$ is in the isogeny class of $G$ or ${ }^{L} G$ but does not necessarily coincide with either of them. The vertex algebra technology provides information about these new dualities as well, and about further refinements which occur when the choice of spin structure on $X$ is removed.
} 
We argue that the compactification functor of (iii) can be constructed rigorously as a localization functor for the vertex algebra $V_{\kappa}^{G}\left(D_{0,1}^{G} \rightarrow B\right)$, where $D_{0,1}^{G} \rightarrow B$ is any non-degenerate junction from the Dirichlet boundary condition $D_{0,1}^{G}$ to $B$.

The notion of localization functor is familiar from representation theory of vertex algebras and $2 \mathrm{~d}$ conformal field theory (see e.g. [25]). It assigns to a module over a vertex algebra with affine Kac-Moody symmetry its sheaf of coinvariants twisted by various $G$-bundles (these are the dual spaces to the spaces of conformal blocks). This sheaf is naturally a twisted $D$-module on $\operatorname{Bun}_{G}$.

(v) We conjecture that morphisms between the $D$-modules associated to objects in $\mathcal{C}_{\kappa}^{G}\left(B_{1}\right)$ and $\mathcal{C}_{\kappa}^{G}\left(B_{2}\right)$ can be identified with the spaces of coinvariants of the corresponding modules over the vertex algebra $V_{\kappa}^{G}\left(B_{1} \rightarrow B_{2}\right)$ for a non-degenerate junction $B_{1} \rightarrow B_{2}$ (for such junctions it follows therefore that the spaces of coinvariants of $V_{\kappa}^{G}\left(B_{1} \rightarrow B_{2}\right)$-modules should be independent of the specifics of the junction $\left.B_{1} \rightarrow B_{2}\right)$.

(vi) For irrational $\kappa$, we conjecture that a kernel of the equivalence $\mathcal{E}_{\kappa}^{G, g}$ given by 1.3 . can be constructed as the sheaf of coinvariants of the vertex algebra

$$
V_{\kappa}^{G}\left(D_{0,1} \rightarrow g\left(D_{0,1}\right)\right)
$$

associated to a non-degenerate junction $D_{0,1} \rightarrow g\left(D_{0,1}\right)$, where $g\left(D_{0,1}\right)$ is the boundary condition in $4 \mathrm{~d}$ gauge obtained by applying the duality $g \in \mathcal{G}_{\kappa}^{G}$ to the Dirichlet boundary condition $D_{0,1}^{G}$. Unlike $D_{0,1}^{G}$, these dual boundary conditions are notoriously difficult to describe directly (see [40, 41]). However, as we explain below, there are various tricks which enable us to construct explicitly various vertex algebras $V_{\kappa}^{G}\left(D_{0,1} \rightarrow g\left(D_{0,1}\right)\right)$ without knowing what $g\left(D_{0,1}\right)$ is. In fact, in this paper we construct two families of such vertex algebras with the favorable property that all of their conformal dimensions (apart from the vacuum) are strictly positive and the graded components corresponding to all conformal dimensions are finite-dimensional.

Although 4d gauge theory is a powerful motivator, many of these results and conjectures can be understood directly and rigorously in terms of the junction vertex algebras and their properties. Thus, the junction vertex algebras enable us to translate subtle and complicated structures of $4 \mathrm{~d}$ gauge theory into a simpler, mathematically rigorous world of vertex algebras, their modules, conformal blocks and localization functors. In this world, the structures relevant to the qGL dualities can be expressed in terms of a kind of lego game, in which building blocks are labeled by the basic boundary conditions and their images under various dualities (a precise nomenclature is set up in Section 3.5).

To each junction between these blocks and a label $(G, \kappa)$, which identifies the bulk $4 \mathrm{~d}$ gauge theory in which the junction is implemented, we assign a vertex algebra. (We want to emphasize that a given pair of boundary conditions may have many different junctions; the corresponding vertex algebras may well be non-isomorphic.) There are several basic junctions for which we know the corresponding vertex algebra at the outset. For instance, a junction from Dirichlet to Neumann gives us an affine Kac-Moody algebra, a junction from 
Nahm to Neumann gives us the corresponding $\mathcal{W}$-algebra, and so on. And then there are two standard moves which enable us to produce new junctions and new vertex algebras.

The first move is composition: we can compose two junctions $B_{1} \rightarrow B_{2}$ and $B_{2} \rightarrow B_{3}$ with label $(G, \kappa)$ to produce a new junction $B_{1} \rightarrow B_{3}$ with the same label. Furthermore, for many junctions, we can construct the corresponding vertex algebra explicitly. For irrational $\kappa$, it is an extension of the tensor product of the vertex algebra associated to $B_{1} \rightarrow B_{2}$ and the vertex algebra $B_{2} \rightarrow B_{3}$ by a specific family of modules.

The second move is duality: the vertex algebras arising in different duality frames of a given junction should be the same. In other words, for each element $g$ of the duality groupoid $\mathcal{G}_{\kappa}^{G}$, the vertex algebra assigned to a junction $B_{1} \rightarrow B_{2}$ with a label $(G, \kappa)$ should be isomorphic to the vertex algebra associated to its duality image $g\left(B_{1}\right) \rightarrow g\left(B_{2}\right)$ with the label $(g(G), g(\kappa))$. If both junction vertex algebras can be constructed explicitly, we obtain an isomorphism between them, which could be non-trivial (such as the duality of $\mathcal{W}$-algebras [22]). More often than not, however, only one of these vertex algebras is known a priori, and then this move gives us a way to define the other vertex algebra.

Thus, we obtain "junction calculus" with two standard moves at our disposal: composition of two junctions produces another junction, and changing the duality frame of a junction gives rise to an equivalent junction. Iterating these moves, we obtain a vast array of junctions, and hence the corresponding vertex algebras, many examples of which are presented below.

Since all these structures arise from the $4 \mathrm{~d}$ gauge theory, we expect that this junction calculus satisfies a kind of bootstrap consistency. In other words, whenever we obtain the same junction by means of different sequences of the standard moves applied to basic junctions, the corresponding vertex algebras should be isomorphic. It is interesting to ask what sort of mathematical structure this represents and what are the minimal requirements for its consistency (as a useful analogy, consider the Kirby calculus). We do not address this question in the present paper, but hope to return to it in a future work. Here we focus on various applications of the rich framework provided by this junction calculus.

The first application has already been mentioned in (vi) above: we can construct junctions $D_{0,1}^{G} \rightarrow g\left(D_{0,1}^{G}\right)$ from Dirichlet to its $g$-dual boundary condition. The corresponding vertex algebra has two commuting affine Kac-Moody algebra symmetries: $\widehat{\mathfrak{g}}$ and $\widehat{\mathfrak{g}^{\prime}}$, where $\mathfrak{g}^{\prime}$ is the $g$-dual of $\mathfrak{g}$ (i.e. it is $\mathfrak{g}$ or ${ }_{\mathfrak{g}}$ depending on what $g$ is). We conjecture that the sheaf of coinvariants of this vertex algebra on $\operatorname{Bun}_{G} \times \operatorname{Bun}_{g(G)}$ is a kernel of the qGL equivalence $\mathcal{E}_{\kappa}^{G, g}$ corresponding to $g$ (see formula $(1.3)$ ).

Our construction of the kernels is automatically compatible with the expected characteristic property of the functor $S_{m}$; namely, that it should relate two important families of $D$-modules:

$$
S_{m}: \mathcal{D}_{\kappa}^{x_{i}, \lambda_{i}} \longleftrightarrow \Psi_{-1 / m \kappa}^{x_{i}, \lambda_{i}}, \quad x_{i} \in X, \quad \lambda_{i} \in P^{+}(G)
$$

which are local modifications of the sheaf $\mathcal{D}_{\kappa}$ of $\kappa$-twisted differential operators on $\operatorname{Bun}_{G}$ and the Whittaker sheaf $\Psi_{-1 / m \kappa}$ on $\operatorname{Bun}_{L_{G}}$ defining a geometric analogue of the Whittaker functional on the space of automorphic functions (the Whittaker sheaf may also be viewed as a quantization the structure sheaf of the oper manifold, which appears in the limit $\kappa \rightarrow 0$ ). 
The second application is that we can express morphisms between various $D$-modules $\mathcal{F}_{1}$ and $\mathcal{F}_{2}$ on $\operatorname{Bun}_{G}$ in terms of conformal blocks of a suitable vertex algebra. The most basic example is when $\mathcal{F}_{1}$ is the $D$-module of $\delta$-functions supported at a point $\mathcal{P} \in \operatorname{Bun}_{G}$ and $\mathcal{F}_{2}$ is in the image of the compactification functor corresponding to a boundary condition $B$. In this case, $\operatorname{Hom}\left(\mathcal{F}_{1}, \mathcal{F}_{2}\right)$ should be isomorphic to the space of $\mathcal{P}$-twisted coinvariants of a module over the vertex algebra obtained from the junction from Dirichlet to $B$.

For example, the fibers of the $D$-modules $\mathcal{D}_{\kappa}^{x_{i}, \lambda_{i}}$ at points of $\operatorname{Bun}_{G}$ are the spaces of coinvariants of the tensor product of the corresponding Weyl modules over the Kac-Moody vertex algebra $V_{\kappa}(\mathfrak{g})$.

As another example, the vector space

$$
\operatorname{Hom}\left(\Psi_{\kappa}^{x_{i}, \mu_{i}^{\vee}}, \mathcal{D}_{\kappa}^{x_{i}, \lambda_{i}}\right)
$$

is expected to be isomorphic to the space of coinvariants of the $\mathcal{W}$-algebra $\mathcal{W}_{\kappa}(\mathfrak{g})$ obtained by the quantum Drinfeld-Sokolov reduction of $V_{\kappa}(\mathfrak{g})$.

A pair of boundary conditions, $B_{1}$ and $B_{2}$ may well have different junctions $B_{1} \rightarrow$ $B_{2}$ which give rise to non-isomorphic vertex algebras. However, we conjecture that the corresponding spaces of coinvariants of modules over these vertex algebras are isomorphic to each other for all non-degenerate junctions. Some of these isomorphisms may be quite non-trivial.

The third application is that we can construct many interesting $D$-modules that are qGL dual to each other. For example, take $G=G L(n)$ or semisimple and self-dual with $m=1$ (this means that $G$ is a product of $E_{8}$ factors). Then the entire group $P S L_{2}(\mathbb{Z})$ is realized by functors $\mathcal{E}_{\kappa}^{G, g}$, and we have a relation $(S T)^{3}=1$. Furthermore, the functor $T$ leaves $\Psi_{\kappa}^{x_{i}, \mu_{i}^{\vee}}$ invariant, which implies that $S T S$ must send $\mathcal{D}_{\kappa}^{x_{i}, \lambda_{i}}$ to $\mathcal{D}_{S T S(\kappa)}^{x_{i}, \lambda_{i}}$. But $S T S=T^{-1} S T^{-1}$. We thus learn (almost for free!) that under the qGL duality $S$,

$$
\mathcal{L}_{G}^{-1} \otimes \mathcal{D}_{\kappa+1}^{x_{i}, \lambda_{i}} \in D_{\kappa}\left(\operatorname{Bun}_{G}\right) \quad \longleftrightarrow \quad \mathcal{L}_{G} \otimes \mathcal{D}_{-\kappa^{-1}-1}^{x_{i}, \lambda_{i}} \in D_{-1 / \kappa}\left(\operatorname{Bun}_{G}\right)
$$

Many non-trivial and perhaps even surprising statements of this kind can be obtained this way.

Finally, we expect that it is possible to obtain a kernel of the Geometric Langlands duality proper as a carefully defined critical level limit $\kappa \rightarrow 0$ of the kernels constructed using the junction calculus (see Section 6.7 for a brief discussion). We leave the details to a future work.

The paper is organized as follows. In Section 2 we give a brief overview of the subject, summarizing the links between boundary conditions in $4 \mathrm{~d}$ gauge theories, vertex algebras, and $D$-modules on $\operatorname{Bun}_{G}$. In Section 3 we focus on a specific class of $4 \mathrm{~d}$ gauge theories, the GL twisted $N=4$ supersymmetric theories defined in [55]. For these theories, we give concrete examples of boundary conditions and categories associated to them, junction vertex algebras, compactification functors, and the action of the duality groupoid. We also formulate conjectures linking conformal blocks of the junction vertex algebras and morphisms between $D$-modules on $\operatorname{Bun}_{G}$ obtained via the compactification functors. In Section 4 we explain how the action of the duality groupoid on the categories of line defects associated to boundary conditions gives rise to qGL dualities between categories of twisted 
$D$-modules on $\operatorname{Bun}_{G}$ and $\operatorname{Bun}_{L_{G}}$. In Section 5 we present an explicit construction of a family of junction vertex algebras $X_{p, q}(G)$ for an arbitrary simple Lie group $G$, whose sheaves of coinvariants we expect to give rise to kernels of specific qGL dualities. For positive $p$ and $q$ with either of them greater than 1, these vertex algebras have the favorable property that conformal dimensions of all fields other than the vacuum are strictly positive.

Section 6 starts with a detailed discussion of the links between the $D$-modules and the branes associated to the basic boundary conditions. We then explain how the compactification functor (with values in twisted $D$-modules on $\mathrm{Bun}_{G}$ ) comes about from the point of view of $4 \mathrm{~d}$ gauge theory and from the point of view of the theory of vertex algebras. In particular, we show that under some natural assumptions it can be constructed as the localization functor for the corresponding junction vertex algebra (up to tensoring with a line bundle on $\operatorname{Bun}_{G}$ ). In Sections 7 and 8 we discuss in more detail various examples of the categories of line defects and junction vertex algebras arising from $4 \mathrm{~d}$ gauge theories with gauge groups $U(1)$ and $S U(2) / S O(3)$, respectively, paying special attention to the spin and gerbe subtleties. These subtleties for general groups are discussed in Section 9. Finally, in Section 10, we produce more examples of kernel vertex algebras for general simple Lie groups. Section 11 lists some open questions and directions for future research.

Acknowledgements. E.F. thanks Joerg Teschner and Edward Witten for valuable discussions. D.G. thanks Alexander Braverman, Kevin Costello, Thomas Creutzig, Dennis Gaitsgory and especially Philsang Yoo for valuable discussions and explanations.

E.F. was supported by NSF grant DMS-1601934. D.G. is supported by the NSERC Discovery Grant program and by the Perimeter Institute for Theoretical Physics. Research at Perimeter Institute is supported by the Government of Canada through Industry Canada and by the Province of Ontario through the Ministry of Research and Innovation.

\section{Overview of the GaUge theOry Setup}

Here we summarize the links between boundary conditions in $4 \mathrm{~d}$ supersymmetric gauge theories, vertex algebras, and $D$-modules on the moduli stack $\operatorname{Bun}_{G}$ of $G$-bundles on a curve $X$. We also discuss the relations between dualities of gauge theories, quantum Langlands dualities, and various operations on vertex algebras. The purpose of this section is to give a bird's-eye view of the subject. Therefore, we only give brief descriptions of some these objects and emphasize the links between them. More details are given in the subsequent sections. We also refer the reader to the earlier works [40, 41, 36, 37, 39, 16].

Useful mathematical groundwork on boundary conditions for $4 \mathrm{~d}$ supersymmetric gauge theories is presented in [19, 14]. Some important links between boundary conditions and the Geometric Langlands Program have been found in (yet unpublished) work by Yoo [69]. That includes, in particular, the characterization (reviewed below) of the categories of boundary line defects at Neumann and Nahm boundary conditions and their junction local operator algebras. The characterization of the category of line defects at Dirichlet boundary conditions was developed by Yoo and one of the authors in the course of an ongoing project on the local Geometric Langlands Program [43].

Several of the statements below have a rather natural TFT interpretation. Despite that, we warn the reader that our setup differs in an important way from the standard TFT setup, 
where one may describe a $4 \mathrm{~d}$ topological theory as some sort of 3-category, with objects associated to topological 3d boundary conditions, morphisms associated to topological $2 \mathrm{~d}$ junctions, 2-morphisms associated to $1 \mathrm{~d}$ junctions of junctions, etc.

Topologically twisted $4 \mathrm{~d}$ gauge theory is equipped with topological $3 \mathrm{~d}$ boundary conditions, which are the main actors in our setup. On the other hand, it admits no topological $2 \mathrm{~d}$ junctions between generic pairs of boundary conditions. Instead, it admits holomorphic $2 \mathrm{~d}$ junctions, which support interesting vertex algebras of local operators. These vertex algebras are our main computational tools ${ }^{4}$

In this paper we do not employ the full toolbox available to us in topologically twisted $4 \mathrm{~d}$ gauge theory. In particular, this toolbox includes topological $3 \mathrm{~d}$ interfaces (domain walls) and topological $2 \mathrm{~d}$ surface defects. These tools are invaluable for uncovering other aspects of qGL dualities: the study of interfaces will enable us to construct functors between the categories of $D$-modules on $\operatorname{Bun}_{G}$ and $\operatorname{Bun}_{H}$, generalizing the geometric Langlands functoriality (see Sect. 4 of [27]); the study of surface defects will allow us to introduce ramification into the picture (generalizing [51]), and to set up a proper framework for the local qGL dualities respectively. We leave these topics for future work.

Our main objective in this paper is to understand the compactification functor associated to a (possibly decorated) Riemann surface $X$. Intuitively, this is the map from structures in the $4 \mathrm{~d}$ gauge theory $\mathcal{T}$ to structures in the effective $2 \mathrm{~d}$ topological theory $\mathcal{T}[X]$ which describes the compactification of $\mathcal{T}$ on $X$.

In the first approximation, this $2 \mathrm{~d}$ theory is a twisted sigma model whose target as the moduli space $\mathcal{M}_{H}(G)$ of solutions of Hitchin's equations [52]. However, this description is incomplete and inadequate for understanding important objects and phenomena in this $2 \mathrm{~d}$ theory, such as boundary conditions. Mathematically, this can be expressed as saying that in the first approximation, the category of $1 \mathrm{~d}$ boundary conditions in the $2 \mathrm{~d}$ theory $\mathcal{T}[X]$ is described by some category of branes on $\mathcal{M}_{H}(G)$, but this description omits important boundary conditions. A better choice is the category of twisted $D$-modules on $\operatorname{Bun}_{G}$, and for irrational values of the coupling constant $\kappa$ (which corresponds to the twisting parameter) we do expect this to be the correct answer. But for rational values of $\kappa$ there are subtle differences between the category of $1 \mathrm{~d}$ boundary conditions in $\mathcal{T}[X]$ and the category of $\kappa$-twisted $D$-modules on $\operatorname{Bun}_{G}$; this can be seen in the fact that some of the $D$-modules may be unphysical because of various issues with the $2 \mathrm{~d}$ theory (see Remark 6.1 below).

In this paper we will mostly sidestep these concerns. Our objective is not to identify specific categories of $\kappa$-twisted $D$-modules on $\operatorname{Bun}_{G}$ for which qGL duality would be an equivalence for all values of $\kappa$. Rather, we want to find an explicit and practical description of the compactification functor mapping decorated boundary conditions of the $4 \mathrm{~d}$ gauge theory and various junctions between them to twisted $D$-modules on $\operatorname{Bun}_{G}$ and morphisms between them.

Once we achieve that, the general machine of $4 \mathrm{~d}$ gauge theory dualities will then allow us to construct specific collections of qGL dual twisted $D$-modules with matching properties

\footnotetext{
${ }^{4}$ The resulting structure is a higher analogue of the structure which arises naturally in the study of $3 \mathrm{~d}$ Chern-Simons theory, which is a topological field theory but typically admits no topological 2d boundary conditions. Instead, it admits holomorphic $2 \mathrm{~d}$ boundary conditions, supporting rational vertex algebras.
} 
as well as a variety of duality "kernels". We expect that studying these data further will ultimately allow us to define the qGL dualities precisely and in full generality.

See Table 1 for a brief summary of the role played by objects in various dimensions.

\begin{tabular}{|c||c||c|}
\hline Gauge Theory & Vertex Algebra & Compactification on $X$ \\
\hline \hline 4d bulk theory $\mathcal{T}_{G}^{\kappa}$ & $?$ & " $D_{\kappa}\left(\right.$ Bun $\left._{G}\right) "$ \\
\hline 3 d boundary & Spin ribbon $($ chiral $)$ & Functor \\
condition $B$ & category $\mathcal{C}(\mathcal{T}, B)$ & $\mathcal{C}(\mathcal{T}, B) \rightarrow D_{\kappa}\left(\operatorname{Bun}_{G}\right)$ \\
\hline 2d junction & Vertex algebra with & Conformal blocks \\
$B_{1} \rightarrow B_{2}$ & $\mathcal{C}_{1}^{\vee} \otimes \mathcal{C}_{2}$ action & map to morphisms \\
\hline
\end{tabular}

TABLE 1. A brief summary of the relations between gauge theory, vertex algebra and quantum Geometric Langlands structures.

2.1. Categories of Boundary Lines. The starting point is the following:

- Each boundary condition $B$ in a 4 d topologically twisted supersymmetric gauge theory $T$ gives rise to a ribbon category (as defined e.g. in [21]) $\mathcal{C}(\mathcal{T}, B)$ of "boundary line defects".

In a more careful treatment, we should replace ribbon categories with chiral categories (see e.g. [62]), which are better suited for algebraic-geometric considerations. Furthermore, in general $\mathrm{C}(\mathcal{T}, B)$ should be a derived, or a DG category. We will mostly ignore this issue because in the examples we consider below (corresponding to the irrational level $\kappa$ ) we can work with the abelian categories.

Upon compactification of $B$ on a Riemann surface $X$, possibly decorated by line defects at points $x_{i} \in X$, we expect to obtain an object in the category of $1 \mathrm{~d}$ boundary conditions for the $2 \mathrm{~d}$ theory $\mathcal{T}[X]$. This map should give rise to a functor from $\mathrm{C}(\mathcal{T}, B) \otimes \mathfrak{C}(\mathcal{T}, B) \otimes \cdots$, with different factors corresponding to different points $x_{i}$, to that category, which is compatible with braiding and fusion.

Without loss of generality, we can focus on a single point $x \in X$ and denote the corresponding compactification functor from $\mathcal{C}(\mathcal{T}, B)$ by $F_{\mathcal{T}}^{B}$.

2.2. Vertex algebra at a junction. Given a $4 \mathrm{~d}$ bulk theory $\mathcal{T}$ and two $3 \mathrm{~d}$ boundary conditions $B_{1}$ and $B_{2}$, we can look for 2 d junctions interpolating from one boundary condition to the other. We denote such a junction as $J_{12}: B_{1} \rightarrow B_{2}$. In general, the same pair of boundary conditions may admit a variety of distinct junctions, with microscopic definitions which may involve various auxiliary holomorphic $2 \mathrm{~d}$ degrees of freedom.

Most of the time, we will suppress the specific choice of junction $J_{12}$ in our notation, unless we want to draw special attention to it.

To these data, we expect to associate a vertex algebra $V\left(\mathcal{T}, B_{1} \rightarrow B_{2}\right)$ of local operators together with a functor

$$
F_{\mathcal{T}, B_{1} \rightarrow B_{2}}: \mathcal{C}\left(\mathcal{T}, B_{1}\right)^{\vee} \otimes \mathcal{C}\left(B_{2}\right) \rightarrow V\left(\mathcal{T}, B_{1} \rightarrow B_{2}\right)-\bmod ,
$$




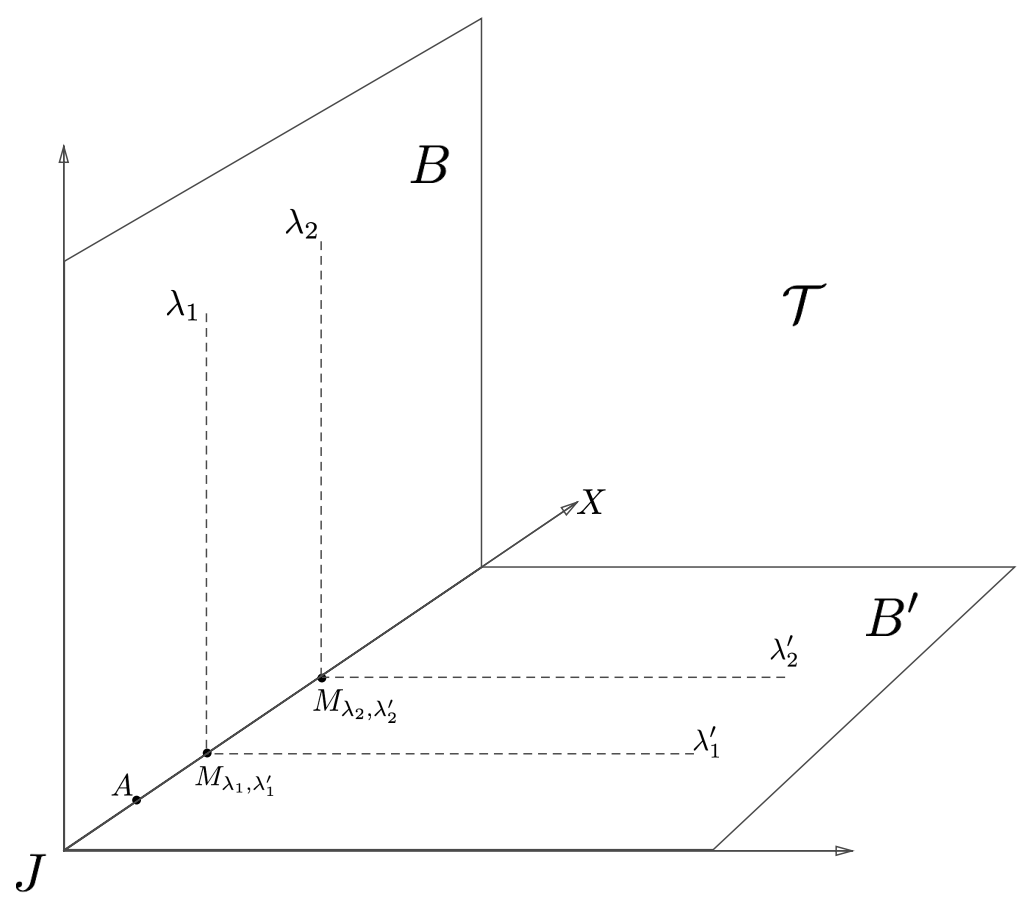

FiguRE 1 . The main physical actors: a $4 \mathrm{~d}$ theory, $3 \mathrm{~d}$ boundary conditions $B$ and $B^{\prime}$, boundary line defects $\lambda_{i}$ and $\lambda_{i}^{\prime}$ placed at $x_{i} \in X$, a $2 \mathrm{~d}$ junction $J$, the vertex algebra $A$ of junction local operators, the $A$-modules $M_{\lambda_{i}, \lambda_{i}^{\prime}}$ of local operators at the endpoints of boundary line defects at the junction. We drew $B$ and $B^{\prime}$ as orthogonal in the topological plane transverse to the junction, but any angle is possible.

where $\mathcal{C}\left(\mathcal{T}, B_{1}\right)^{\vee}$ is the dual category to $\mathcal{C}\left(\mathcal{T}, B_{1}\right) !^{5}$

Physically, the functor maps boundary lines to the spaces of local operators supported at points where the lines end at the junction. These naturally form a module for the vertex algebra $V\left(\mathcal{T}, B_{1} \rightarrow B_{2}\right)$ of local operators supported at generic points of the junction. See Figure 1 for an illustration of these ideas.

This functor is not an equivalence in general, but we expect it to be fully faithful in many interesting cases. If is it fully faithful, we will call the junction non-degenerate.

2.3. Conformal blocks. It is natural to consider conformal blocks (more precisely, their dual spaces called spaces of coinvariants, see [28, 12] and Section 6 below) of a junction vertex algebra $V\left(\mathcal{T}, B_{1} \rightarrow B_{2}\right)$, possibly involving the $V\left(\mathcal{T}, B_{1} \rightarrow B_{2}\right)$-modules associated to the corresponding ribbon categories.

\footnotetext{
${ }^{5}$ In special situations, the bulk $4 \mathrm{~d}$ theory may admit non-trivial category $\mathcal{S}(\mathcal{T})$ of bulk line defects, with functors to the categories of boundary lines. If that's the case, the product should taken over this category. Also, it might be necessary to consider here the dual of the derived, or DG, categories.
} 
We will get such a space of conformal blocks for any choice of curve $X$, possibly decorated by modules placed at one or more points $x_{i} \in X$. By construction, the operation of taking conformal blocks (or coinvariants) gives a functor from the category of $N$-tuples of $V\left(\mathcal{T}, B_{1} \rightarrow B_{2}\right)$-modules to vector spaces, where $N$ is the number of points. In what follows, we restrict ourselves to the case $N=1$, but a generalization to $N>1$ is straightforward.

What interests us the most is that there is a direct link between morphisms between the images of the compactification functor and conformal blocks of the corresponding $V_{\kappa}^{G}\left(B_{1} \rightarrow\right.$ $B_{2}$ )-module.

More precisely, let $\mathcal{A}_{1} \in \mathrm{Ob}\left(\mathcal{C}\left(\mathcal{T}, B_{1}\right)\right), \mathcal{A}_{2} \in \mathrm{Ob}\left(\mathcal{C}\left(\mathcal{T}, B_{2}\right)\right)$. Then on the one hand, these objects can be sent by the compactification functors to objects $\overline{\mathcal{A}}_{1}=F_{\mathcal{T}}^{B_{1}}\left(\mathcal{A}_{1}\right)$ and $\overline{\mathcal{A}}_{2}=F_{\mathcal{T}}^{B_{2}}\left(\mathcal{A}_{2}\right)$ of the category of $1 \mathrm{~d}$ boundary conditions for $\mathcal{T}[X]$.

On the other hand, $F_{\mathcal{T}, B_{1} \rightarrow B_{2}}\left(\mathcal{A}_{1}^{\vee} \otimes \mathcal{A}_{2}\right)$ is a $V\left(B_{1} \rightarrow B_{2}\right)$-module.

Conjecture 2.1. Suppose that the functor $F_{\mathcal{T}, B_{1} \rightarrow B_{2}}$ is fully faithful. Then $\operatorname{Hom}\left(\overline{\mathcal{A}}_{1}, \overline{\mathcal{A}}_{2}\right)$ is isomorphic to the space of coinvariants of the $V\left(B_{1} \rightarrow B_{2}\right)$-module $F_{\mathcal{T}, B_{1} \rightarrow B_{2}}\left(\mathcal{A}_{1}^{\vee} \otimes \mathcal{A}_{2}\right)$.

This is the main tool we will employ to reconstruct the compactification functor.

We also have the following

Conjecture 2.2. Suppose that the functor $F_{\mathcal{T}, B_{1} \rightarrow B_{2}}$ is fully faithful. Then the category of $V\left(B_{1} \rightarrow B_{2}\right)$-modules and the corresponding spaces of coinvariants (and conformal blocks) depend only on $B_{1}$ and $B_{2}$ and not on the junction data between them.

In Sections 3.8 and 6.5 we will explain the significance of these conjectures.

The physical interpretation of these conjectures is straightforward. It involves the space of states for the theory on a space manifold of the form $[0,1] \times X$, with boundary conditions $B_{1}$ and $B_{2}$ at the endpoints of the segment.

By definition, the space of states computes the Hom in the category of boundary conditions in $\mathcal{T}[X]$. It can also be interpreted as the space of states of the $3 \mathrm{~d}$ TFT resulting from compactification of $\mathcal{T}$ on the segment.

The $2 \mathrm{~d}$ junction descends to a boundary condition for such a TFT. Our conjectures can then be seen as a variant of the standard relation between the space of states of a $3 \mathrm{~d}$ TFT and the space of conformal blocks of its boundary vertex algebras. See Figure 2 for an illustration of this setup.

Remark 2.1. We can illustrate such a relation in further detail for the simplest situation namely, a $3 \mathrm{~d}$ TFT $T[C]$ described by some modular tensor category $C$ and a $2 \mathrm{~d}$ rational vertex algebra $A$. This is not an example which occurs in our setup (except when describing some useful auxiliary degrees of freedom in later sections) but it is nevertheless instructive.

Any rational vertex algebra $A$ gives rise to a modular tensor category $A$-mod. That means $A$ can always be found at a boundary for the $3 \mathrm{~d}$ TFT $T[A-\bmod ]$ defined by $A-\bmod$. The space of states of $T[A-\bmod ]$ on a Riemann surface $X$ coincides with the space of conformal blocks of $A$ on $X$.

However, the vertex algebra $A$ can also be found as the algebra of boundary local operators at boundaries of other $3 \mathrm{~d}$ TFTs. The MTC $A-\bmod$ has a universal property: any 


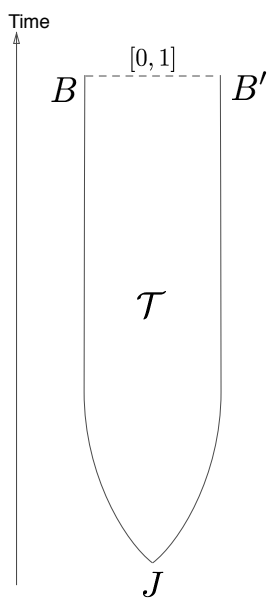

Figure 2. The space-time justification for relating conformal blocks of junction vertex algebras and the Hilbert space of states on $[0,1] \times X$ with $B$ and $B^{\prime}$ boundary conditions: the junction creates states in the the Hilbert space. The $X$ holomorphic direction is not depicted.

boundary condition for $T[C]$ supporting $A$ can be factored as the composition of the canonical boundary condition for the $T[A-\bmod ] 3 \mathrm{~d}$ TFT and a topological interface from $T[C]$ to $T[A-\bmod ]$.

In particular, the functor $C \rightarrow A-\bmod$ from lines to local operators can be reinterpreted as, or factored through, the functor which describes the action of such an interface on the lines of the 3d TFTs. The interface, though, also controls the relation between the spaces of states for the two 3d TFTs on any Riemann surface.

Hence in this basic setup one can derive sharp statements relating the properties of a functor $C \rightarrow A-\bmod$ and the relation between the spaces of states of $T[C]$ and conformal blocks of $A$.

2.4. Boundary conditions with global symmetries. A final ingredient is the observation that certain boundary conditions are equipped with a non-trivial group of global symmetries, which is defined independently of the gauge symmetry of the bulk theory.

Whenever boundary condition $B$ has a non-trivial global symmetry group $H$, the compactification functor can be modified by coupling the system to an $H$-bundle on $X$. This gives families of $1 \mathrm{~d}$ boundary conditions for $\mathcal{T}[X]$ parametrized by $\mathrm{Bun}_{H}$.

Furthermore, junctions of the form $B \rightarrow B^{\prime}$ or $B^{\prime} \rightarrow B$ which preserve the $H$ symmetry will support vertex algebras with an $H$-symmetry implemented by an $\widehat{\mathfrak{h}}$ Kac-Moody subalgebra. As a consequence, spaces of coinvariants can be promoted to twisted $D$-modules on $\operatorname{Bun}_{H}$.

Morphisms between these $D$-modules can be used to give a concrete realization for the spaces of morphisms between the families of $1 \mathrm{~d}$ boundary conditions parametrized by $\mathrm{Bun}_{H}$.

Each 4d gauge theory with gauge group $G$ comes equipped with a special family of "Dirichlet" boundary conditions with global symmetry $G$. These will be key to giving a 
precise formulation of the compactification functors with values in the categories of twisted $D$-modules on $\operatorname{Bun}_{G}$ (see Section 6.3).

2.5. Composition of junctions. Consider now three boundary conditions $B_{1}, B_{2}$ and $B_{3}$ in a bulk theory $\mathcal{T}$, with pairwise junctions $J_{12}$ and $J_{23}$. On physical grounds, we then expect to have a new junction $J_{12} \circ J_{23}$ from $B_{1}$ to $B_{3}$.

The corresponding vertex algebra $V\left(\mathcal{T}, B_{1} \rightarrow B_{3}\right)$ in which we take as junction data the composition of the data $J_{12}$ and $J_{23}$ is expected to include the tensor product vertex algebra

$$
V\left(\mathcal{T}, B_{1} \rightarrow B_{2}\right) \otimes V\left(\mathcal{T}, B_{2} \rightarrow B_{3}\right) .
$$

Furthermore, conjecturally, $V\left(\mathcal{T}, B_{1} \rightarrow B_{3}\right)$ can be built as an extension of the latter by a (super)algebra object $\mathcal{A}_{13}$ of the category

$$
\left[V\left(\mathcal{T}, B_{1} \rightarrow B_{2}\right) \otimes V\left(\mathcal{T}, B_{2} \rightarrow B_{3}\right)\right]-\bmod
$$

which is the image of $\operatorname{Id}_{1}^{\vee} \times \operatorname{Diag} \times \operatorname{Id}_{3}$ under $F_{\mathcal{T}, B_{1} \rightarrow B_{2}} \otimes F_{\mathcal{T}, B_{2} \rightarrow B_{3}}$. Here $\operatorname{Id}_{1}^{\vee}$ and $\operatorname{Id}_{3}$ denote the identity objects in $\mathcal{C}\left(\mathcal{T}, B_{1}\right)^{\vee}$ and $\mathcal{C}\left(\mathcal{T}, B_{3}\right)$, respectively, while Diag is a "diagonal object" in $\mathfrak{C}\left(\mathcal{T}, B_{2}\right) \otimes \mathfrak{C}\left(\mathcal{T}, B_{2}\right)^{\vee}$. This object is easy to define if $\mathfrak{C}\left(\mathcal{T}, B_{2}\right)$ is semisimple as an abelian category. In general, the construction of Diag requires special care.

The composition of the obvious map

$$
\mathrm{C}\left(\mathcal{T}, B_{1}\right)^{\vee} \otimes \mathfrak{C}\left(\mathcal{T}, B_{3}\right) \rightarrow \mathcal{C}\left(\mathcal{T}, B_{1}\right)^{\vee} \otimes \operatorname{Diag} \otimes \mathfrak{C}\left(\mathcal{T}, B_{3}\right)
$$

and $F_{\mathcal{T}, B_{1} \rightarrow B_{2}} \otimes F_{\mathcal{T}, B_{2} \rightarrow B_{3}}$ gives a functor to $\mathcal{A}_{13}-\bmod$ and thus to $V\left(\mathcal{T}, B_{1} \rightarrow B_{3}\right)$-mod, as needed.

See Figure 3 for the physical explanation of this prescription.

In the rest of the paper, we will discuss several such compositions. A particularly important application of these compositions is to produce junctions between boundary conditions which do not simultaneously admit weakly-coupled descriptions, in a sense we will explain momentarily.

2.6. Dualities. A duality is an equivalence between different definitions of the same $4 \mathrm{~d}$ theory. The quantum Geometric Langlands dualities are expected to relate the different definitions (in other words, different duality frames) of the category of $1 \mathrm{~d}$ boundary conditions for $\mathcal{T}[X]$.

Each definition of a theory will come with some collection of "weakly coupled" boundary conditions and junctions, which can be defined microscopically in terms of the fields of the theory and possibly other auxiliary fields. These are the boundary conditions and junctions for which direct calculations of the categories of line defects and vertex algebras are usually possible.

A duality rarely identifies two weakly coupled boundary conditions. More often, it maps weakly coupled boundary conditions to other "strongly coupled" ones for which direct calculations are not feasible.

We will often face the problem to define and compute properties of junctions between boundary conditions which are not simultaneously weakly coupled. For example, in order to study the qGL dualities, we need to employ duality images of the Dirichlet boundary conditions. 


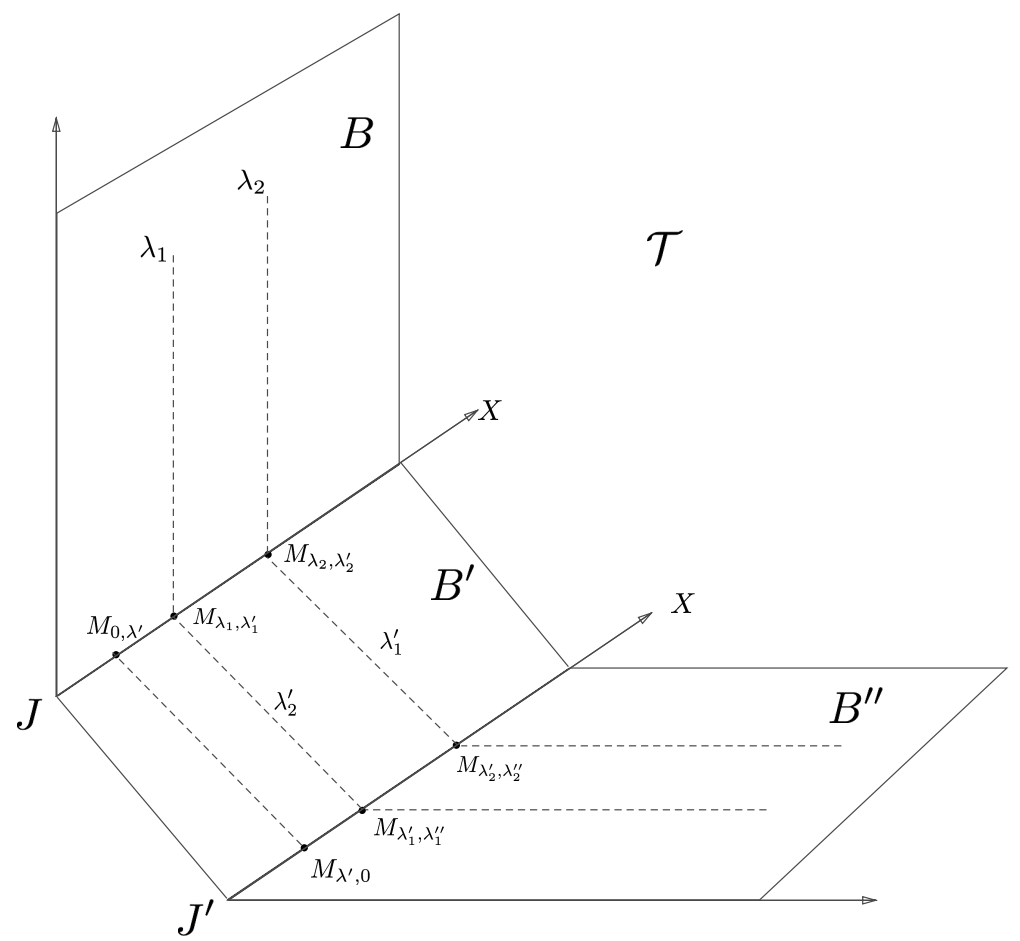

Figure 3. Composition of junctions $J: B^{\prime} \rightarrow B$ and $J^{\prime}: B^{\prime \prime} \rightarrow B^{\prime}$. The local operators for the composite junction can arise from finite segments of line defects in the intermediate boundary. Thus the new junction vertex algebra is an extension of $A \times A^{\prime}$ by a combination of product of modules $M_{0, \lambda^{\prime}} \times M_{\lambda^{\prime}, 0}$. Modules for the new vertex algebra are similarly built from combinations of the form $M_{\lambda, \lambda^{\prime}} \times M_{\lambda^{\prime}, \lambda^{\prime \prime}}$.

Our solution to the problem is simple: we find a chain of boundary conditions such that each consecutive pair is simultaneously weakly coupled in some duality frame of the $4 \mathrm{~d}$ theory; find and compute the properties of the corresponding junctions; and then compose these junctions into a single junction between the first and the last boundary conditions in the chain.

2.7. Spin subtleties. We should mention here an important subtlety which will be recurring in our analysis. A priori, the constructions we employ involve theories and defects which are spin-topological or spin-holomorphic: they are defined only on manifolds equipped with a spin structure. In many situations, though, we can refine the constructions so that the corresponding objects become truly topological or holomorphic. 
Working with "spin" constructions is somewhat simpler. For example, the dualities of gauge theories are well understood in the spin case, while the non-spin refinement of the dualities is only known in some examples ${ }^{6}$

The most immediate consequence of working with spin constructions is that boundary line defects form spin-ribbon categories, a variant of ribbon categories in which the topological twisting of an object is defined only up to an integral multiple of $\pi$ (rather than $2 \pi$ ). Spin constructions also employ spin-vertex algebras, i.e. vertex algebras in which some of the fields have half-integral spin. Conformal blocks of modules over the spin-vertex algebras are only well-defined on a complex curve equipped with a specific choice of spin structure (i.e. a square root of the canonical line bundle).

If a spin-vertex algebra can be coupled to $H$-bundles and $H$ has a $\mathbb{Z}_{2}$ central subgroup which acts as -1 on the fields of half-integral spin, then instead of choosing a spin structure, we can work with $\operatorname{Spin}_{H}$ bundles rather than $G$-bundles (we could say we have a $\operatorname{Spin}_{H}$ vertex algebra) in this case). Conformal blocks then become dependent on $\operatorname{Spin}_{H}$ bundles, and they give rise to twisted $D$-modules on the stack $B_{\operatorname{Spin}_{H}}$ of such bundles. See Section 7.4 for more details, including the definition of $\operatorname{Spin}_{H}$ bundles.

Finally, notice that "spin" and "super" are distinct notions. Our vertex algebras are allowed to be vertex superalgebras, in the sense of including odd (Grassmann) fields. But these fields do not necessarily have half-integral spin, and conversely, fields of half-integral spin may or may not be odd.

\section{Boundary COnditions, DuAlities, And Junction vertex ALGEBras}

In this section we discuss specific boundary conditions and junctions between them. Though they exist universally in all twisted $4 \mathrm{~d}$ gauge theories, we will focus on the GL twisted $4 \mathrm{~d}$ gauge theory, as defined in [55], with a compact connected gauge group $G_{c}$, whose complexification is denoted by $G$ (this is a connected reductive Lie group over $\mathbb{C}$ ), and the topological coupling constant which is denoted by $\Psi$ in [55], but which we will denote by $\kappa \cdot 7$ Upon compactification of the $4 \mathrm{~d}$ gauge theory on a Riemann surface $X$, we naturally obtain a functor from the category of line defects associated with a given boundary condition to the category $D_{\kappa}\left(\operatorname{Bun}_{G}\right)$ of twisted $D$-modules on the moduli stacks $\operatorname{Bun}_{G}$ of $G$-bundles on $X$. We will call it the compactification functor. In subsequent sections, we will see how these functors, together with the action of quantum dualities on

\footnotetext{
${ }^{6}$ As the physical gauge theory contains spinors, the theory is initially defined only on spin manifolds and the duality group(oid) has been studied in detail only on such manifolds. The topologically twisted theory, however, has no spinors and can be readily defined on a manifold without a spin structure. But then the duality group(oid) needs to be modified in ways that are only partly understood in the existing literature.

As the spinors in the physical theory transform in the fundamental representation of the $S U(4)_{R} \simeq$ $\operatorname{Spin}(6)_{R}$ R-symmetry group, while fields of integral spin transform in the representations of $S O(6)_{R}$, it should be possible to couple the theory to $\operatorname{Spin}_{S U(4)_{R}}$-bundles, i.e. bundles on a four-manifold $M$ with the structure group $(\operatorname{Spin}(4) \times S U(4)) / \mathbb{Z}_{2}\left(\right.$ which is a $\mathbb{Z}_{2}$-extension of the structure group $S O(4) \times\left(S U(4) / \mathbb{Z}_{2}\right)=$ $S O(4) \times S O(6))$, such that the corresponding $S O(4) \times\left(S U(4) / \mathbb{Z}_{2}\right)$-bundle has the frame bundle of $M$ along the first factor. The latter choice is probably better in preparation for a topological twist. It suggests that the spin-refined duality action may already be probed within the physical theory.

${ }^{7}$ There is in fact a separate coupling for each simple factor of the Lie algebra of $G$, together with a matrix of couplings for Abelian factors.
} 
the boundary conditions and junctions between them, naturally lead us to valuable insights into the quantum Geometric Langlands theory.

In fact, there is a larger class of theories that we need to consider in order to fully explore the duality groups of gauge theories. These theories include extra "discrete theta angles". For now, we set the discrete theta angles to 0 , but we will come back to the more general theories in later sections.

The parameter $\kappa$ of gauge theory may be viewed as the level of the corresponding affine Kac-Moody algebra $\widehat{\mathfrak{g}}$ (the affinization of the Lie algebra $\mathfrak{g}$ of $G$ ) shifted by the critical value, or as the twisting parameter for $D$-modules on the affine Grassmannian $\operatorname{Gr}_{G}$ and on $\operatorname{Bun}_{G}$, also shifted by the critical value (corresponding to the properly defined square root of the canonical line bundle). We will denote this bulk theory by $\mathcal{T}_{\kappa}^{G}$.

The properties of the theory $\mathcal{T}_{\kappa}^{G}$ are rather uniform as a function of $\kappa$, as long as $\kappa$ is not rational. A variety of new phenomena occurs for rational values of $\kappa$, such as the existence of a non-trivial category $\mathcal{S}\left(\mathcal{T}_{\kappa}^{G}\right)$ of bulk line defects. For simplicity, unless specified otherwise, we will assume throughout this paper that $\kappa$ is irrational (i.e. $\kappa \in \mathbb{C} \backslash \mathbb{Q}$ ).

3.1. Examples of boundary conditions. In the examples below, we consider basic boundary conditions in the theory $\mathcal{T}_{\kappa}^{G}$ and the ribbon categories corresponding to them, which we will denote by $\mathcal{C}_{\kappa}^{G}(B)$.

(i) The Dirichlet boundary condition. The corresponding category $\mathcal{C}_{\kappa}^{G}(B)$ is the category $D_{-\kappa}\left(\mathrm{Gr}_{G}\right)$ of right $(-\kappa)$-twisted $D$-modules on the affine Grassmannian $\operatorname{Gr}_{G}=G((z)) / G[[z]]$. Recall that $\mathrm{Gr}_{G}$ is an ind-scheme, a union of its closed subschemes of finite type (which are proper finite-dimensional complex algebraic varieties). By definition, the support of each object of $D_{-\kappa}\left(\mathrm{Gr}_{G}\right)$ should belong to one of those subschemes (see e.g. [29] for a precisely definition).

(ii) The Neumann boundary condition. The corresponding category $\mathcal{C}_{\kappa}^{G}(B)$ is the Kazhdan-Lusztig category $\mathrm{KL}_{\kappa}(G)$ [57] of finitely generated $\widehat{\mathfrak{g}}_{\kappa}$-modules $M$ on which the action of the Lie subalgebra $\mathfrak{g}[[z]] \subset \widehat{\mathfrak{g}}_{\kappa}$ is locally finite and can be exponentiated to an action of $G[[z]]$ (equivalently, $M$ decomposes into a direct sum of finite-dimensional modules over the constant Lie subalgebra $\mathfrak{g}$ which can be exponentiated to an action of $G$, and for any vector $v \in M$, there exists $n>0$ such that $\left.\mathfrak{g} \otimes t^{n} \mathbb{C}[[t]] \cdot v=0\right)$.

(iii) The principal Nahm boundary condition. The corresponding category $\mathcal{C}_{\kappa}^{G}(B)$ is the Whittaker category Whit $-\kappa(G)$, of $(-\kappa)$-twisted $D$-modules on $\operatorname{Gr}_{G}$ which are $N((z)$ )-equivariant with respect to a fixed non-degenerate character $\psi$ (corresponding to the principal element appearing in the Nahm boundary condition). Here $N$ is the unipotent subgroup of $G .^{8}$ See [31, 45] for the precise definition. The chiral category structure is defined in [13, 61].

\footnotetext{
${ }^{8}$ The category Whit $_{-\kappa}(G)$ is an example of a category that requires, for some $G$, a choice of spin structure, i.e. a specific choice of a square root of the canonical line bundle on a formal disc (or on a curve $X$ ). Indeed, one needs to make this choice in order to define a non-degenerate character on the group $N((z))$ in a coordinate-independent fashion.
} 
(iv) Other Nahm boundary conditions. There is a more general family of boundary conditions labeled by the extra data of a conjugacy class of an embedding $\rho$ of $\mathfrak{s l}_{2}$ into $\mathfrak{g}$ (or equivalently, a non-zero nilpotent conjugacy class in $\mathfrak{g}$ ). The corresponding category Whit ${ }_{-\kappa}^{\rho}(G)$ can be defined in a similar manner as for the principal Nahm boundary condition.

In addition, we expect that each of these categories is equipped with a functor:

- For each boundary condition $B$ in the theory $\mathcal{T}_{\kappa}^{G}$ and every compact Riemann surface (smooth projective curve) $X$ with a point $x \in X$, there is a compactification functor $F_{\kappa}^{G, B}$ from the category $\mathcal{C}_{\kappa}^{G}(B)$ to the category $D_{\kappa}\left(\operatorname{Bun}_{G}\right)$ of left $\kappa$-twisted $D$-modules on $\operatorname{Bun}_{G}$. In general, this is a functor between derived, or DG, categories. This functor has a multi-point generalization compatible with fusion and braiding in $\mathcal{C}_{\kappa}^{G}(B)$.

- The image $F_{\kappa}^{G, B}(I)$ of the identity object $I$ in $\mathcal{C}_{\kappa}^{G}(B)$ does not depend on the point $x$. We call the map from boundary conditions to twisted $D$-modules on $\operatorname{Bun}_{G}$,

$$
B \mapsto F_{\kappa}^{G, B}(I)
$$

the compactification map.

In Section 6, under some natural assumptions, we will describe the compactification functor as a localization functor for a certain vertex algebra with affine Kac-Moody symmetry. We will also discuss the connection between the corresponding twisted $D$-modules on $B_{G}$ and branes on the Hitchin moduli space $\mathcal{M}_{H}(G)$ associated to $X$ constructed in [55]. Here we will only mention that the boundary conditions available at general $\kappa$ are non-trivial deformations of standard half-BPS boundary conditions of the physical theory. Half-BPS boundary conditions can be employed either at $\kappa=0$ or at $\kappa=\infty$, but only a few can be deformed to other values of $\kappa$. Neumann are deformed from $\kappa=\infty$, while Dirichlet and Nahm are deformed from $\kappa=0$.

The compactification functor can be interpreted as the composition of two functors: a compactification of boundary conditions to branes and a functor from branes to twisted $D$-modules. The brane interpretation is useful and instructive, but we stress that the compactification functors can be defined directly, i.e. bypassing the categories of branes on $\mathcal{M}_{H}(G)$ introduced in [55]. See Sections 6.1 and 6.2 for more details.

This simplifies some aspects of the construction. For example, as explained in [55], the sigma model description is simplest for real values of $\kappa$, but for other values of $\kappa$ one needs to consider generalized complex structures on $\mathcal{M}_{H}(G)$. On the other hand, the categories of $\kappa$-twisted $D$-modules on $\operatorname{Bun}_{G}$ behave in a more uniform way relative to $\kappa$.

3.2. Examples of the compactification functors. We now describe examples of the compactification functors, as well as the images of the standard objects in $\mathcal{C}_{\kappa}^{G}(B)$ under these functors. We also comment on the corresponding branes on the Hitchin moduli space $\mathcal{M}_{H}(G, X)$. 
(i) The Dirichlet boundary condition. The compactification functor is the suitably defined push-forward corresponding to the surjective map

$$
\mathrm{Gr}_{G} \rightarrow \operatorname{Bun}_{G}=G(X \backslash x) \backslash \operatorname{Gr}_{G} .
$$

Note that under this functor right $(-\kappa)$-twisted $D$-modules on $\operatorname{Gr}_{G}$ are mapped to left $\kappa$-twisted $D$-modules on $\operatorname{Bun}_{G}$.

The standard objects in the category $D_{-\kappa}\left(\mathrm{Gr}_{G}\right)$ are " $\delta$-function" (right) $(-\kappa)$ twisted $D$-modules supported at points of $\operatorname{Gr}_{G}$ (see below), which we denote by $\delta_{p}^{-\kappa}, p \in \mathrm{Gr}_{G}$. The corresponding $D$-module on $\mathrm{Bun}_{G}$ is the " $\delta$-function" (left) $\kappa$-twisted $D$-module $\delta_{\mathcal{P}}^{\kappa}$, where $\mathcal{P}$ is the image of $p \in \mathrm{Gr}_{G}$ in $\operatorname{Bun}_{G}$. (If $\mathcal{P}$ has non-trivial automorphisms, then the category of twisted $D$-modules supported at $\mathcal{P}$ is non-trivial, but we ignore this for now. See the discussion at the end of this subsection, as well as Remark 6.2.)

For irrational $\kappa$, the $D$-module $\delta_{\mathcal{P}}^{\kappa}$ is a counterpart of the $(A, B, A)$ brane $\mathcal{F}_{\mathcal{P}}^{\prime}$. These are supported on the fibers of the maps from an open subset of $\mathcal{M}_{H}(G, X)$, seen as a moduli space of flat $G$-bundles, to $\mathcal{M}(G, X)$, the moduli space of semistable $G$-bundles on $X$ (see Section 6.1 for more details).

These branes are deformations of the $(B, A, A)$ branes $F_{\mathcal{P}}$, the fibers of the projection of $\mathcal{M}_{H}(G, X)$, seen as a moduli space of Higgs bundles on $X$, to $\mathcal{M}(G, X)$. These originate from the undeformed Dirichlet boundary conditions at $\kappa=0$.

(ii) The Neumann boundary condition. The compactification functor is the localization functor $\Delta_{\kappa}$ (see [28, Ch. 18, 25] and Section 6 below).

The standard objects in $\mathrm{KL}_{\kappa}(G)$ are the Weyl modules $\mathbb{V}_{\lambda, \kappa}$, where $\lambda \in P^{+}(G)$, the set of dominant integral weight of $G$ (i.e. highest weights of irreducible representations of $G$ ). (Note that for $\kappa \notin \mathbb{Q}, \mathrm{KL}_{\kappa}(G)$ is a semisimple category with each object isomorphic to the direct sum of finitely many Weyl modules $\mathbb{V}_{\lambda, \kappa}$.) The corresponding (left) twisted $D$-module $\Delta_{\kappa}\left(\mathbb{V}_{\lambda, \kappa}\right)=\mathcal{D}_{\kappa}^{x, \lambda}$ on $\operatorname{Bun}_{G}$ is the tensor product $\mathcal{D}_{\kappa} \otimes_{\mathcal{O}} \mathcal{V}_{x, \lambda}$, where $\mathcal{D}_{\kappa}$ is the sheaf of $\kappa$-twisted differential operators on $\operatorname{Bun}_{G}$ and $\mathcal{V}_{x, \lambda}$ is the sheaf of sections of the tautological vector bundle on $\operatorname{Bun}_{G}$ corresponding to the finite-dimensional representation $V_{\lambda}$ of $G$ and the point $x$ of the curve $X$.

In particular, for the vacuum module $\mathbb{V}_{0, \kappa}$ we have $\Delta_{\kappa}\left(\mathbb{V}_{0, \kappa}\right)=\mathcal{D}_{\kappa}$. This is the image of the compactification map.

The brane on $\mathcal{M}_{H}(G, X)$ corresponding to $\mathcal{D}_{\kappa}$ for irrational $\kappa$ is the canonical coisotropic brane $\mathcal{B}_{\text {c.c. }}$ (see Section 6.1). This is a deformation away from $\kappa=$ $\infty$ of the space-filling $(B, B, B)$ brane $\mathcal{B}$ associated to the undeformed Neumann boundary conditions.

(iii) The (principal) Nahm boundary condition. Consider for simplicity the case that $G$ is simple. Then the Whittaker category Whit $_{-\kappa}(G)$ can be realized as a direct limit of the categories of Whittaker sheaves (left $\kappa$-twisted $D$-modules) on the moduli stacks $\overline{\operatorname{Bun}}_{N}^{\mathcal{F}_{T_{0}}\left(\mu^{\vee} \cdot x\right)}$ of $B$-bundles on $X$, where $\mu^{\vee} \in{ }^{L} P^{+}$, the set of dominant integral coweights of $G$ (see [31]). For each of these categories, we take the functor 
of direct image corresponding to the natural maps $j_{x, \mu^{\vee}}: \overline{\operatorname{Bun}}_{N}^{\mathcal{F}_{T_{0}}\left(\mu^{\vee} \cdot x\right)} \rightarrow \operatorname{Bun}_{G}$. The compactification functor from Whit ${ }_{-\kappa}(G)$ to the category of $\kappa$-twisted $D$ modules on $\operatorname{Bun}_{G}$ is naturally "glued" from these.

The standard objects of Whit $-\kappa(G)$ are the Whittaker sheaves $\Psi_{\kappa}^{x, \mu^{\vee}}, \mu^{\vee} \in{ }^{L} P^{+}$. In particular, the $\mathcal{D}_{\kappa}$-module corresponding to $\Psi_{\kappa}^{x, 0}$ is constructed as follows. Let $\mathcal{F}_{T}^{0}$ be a $T$-bundle on $X$ (where $T$ is the Cartan subgroup of $G$ ) corresponding to the canonical line bundle $K_{X}$ on $X$ and a cocharacter $\check{\rho}$. Note that we may need to choose a square root of the canonical line bundle on $X$ to make sense of this $T$ bundle 9 Let $\overline{\operatorname{Bun}}_{N}^{\mathcal{F}_{T}^{0}}$ be the corresponding moduli stack and $j_{x, 0}$ the corresponding map $\overline{\operatorname{Bun}}_{N}^{\mathcal{F}_{T}^{0}} \rightarrow \operatorname{Bun}_{G}$. It is equipped with a natural map $e v: \operatorname{Bun}_{N}^{\mathcal{F}_{T}^{0}} \rightarrow \mathbb{A}^{1}$, the affine line. Let $\mathcal{E}$ be the $D$-module on $\mathbb{A}^{1}$ generated by the exponential function. Then, $\Psi_{\kappa}^{x, 0}$ is a generalization to arbitrary $\kappa$ of $j_{x, 0 !}\left(e v^{*}(\varepsilon)\right)$. The construction of the other $\mathcal{D}_{\kappa}$-modules $\Psi_{\kappa}^{x, \mu^{\vee}}$ is similar (for $\kappa=0$, they can be obtained by applying the Hecke functors to $\left.\Psi_{0}^{x, 0}\right)$. See [31, 45, 13, 61] for more details.

For $\kappa \neq 0$, the $(A, B, A)$ brane corresponding to $\Psi_{\kappa}^{0, x}$ is the brane of opers. This is a deformation away from $\kappa=0$ of the $(B, A, A)$-brane of "classical opers" (also known as the Hitchin section) associated to undeformed Nahm boundary conditions. See Section 6.1 for more details.

\begin{tabular}{|c||c||c|}
\hline Boundary Condition & Category of Lines & Compactification Functor \\
\hline \hline Dirichlet $\left(D_{0,1}\right)$ & $D_{-\kappa}\left(\mathrm{Gr}_{G}\right)$ & Push-Forward $\mathrm{Gr}_{G} \rightarrow \mathrm{Bun}_{G}$ \\
\hline Neumann $\left(N_{1,0}\right)$ & Kazhdan-Lusztig $\mathrm{KL}_{\kappa}$ & Localization functor $\Delta_{\kappa}$ \\
\hline Principal Nahm $\left(N_{0,1}\right)$ & Whittaker Whit ${ }_{-\kappa}(G)$ & Direct image \\
\hline
\end{tabular}

TABLE 2. The basic boundary conditions that exist universally for all $G$

A few comments on the definition of the sheaf of " $\delta$-functions" supported at a point. Let $Z$ be a smooth algebraic variety over $\mathbb{C}$ (or an ind-scheme such as $\mathrm{Gr}_{G}$ ) and $p$ a point of $Z$. By definition,

$$
\delta_{p}=i_{p !}\left(\mathbb{C}_{p}\right)=\mathcal{D}_{Z} \underset{\mathcal{O}_{Z}}{\otimes} \mathbb{C}_{p}
$$

is the (left) $D$-module push-forward of the constant sheaf at a point (viewed as a $D$-module at that point) under the embedding $i_{p}:$ pt $\rightarrow Z$. Concretely, for any open subset $U \subset M$ containing $p$, we have

$$
\delta_{p}(U)=\mathcal{D}(U) \underset{\mathcal{O}(U)}{\otimes} \mathbb{C}_{p}
$$

\footnotetext{
${ }^{9}$ This is an example of how we may be forced to make a choice of a spin structure on $X$. Once we make such a choice, there is a parameter for $\mathcal{F}_{T}^{0}$ : an element of $H^{1}(X, Z(G))$. For simplicity, we will assume here that $G$ is of adjoint type so that its center $Z(G)$ is trivial.
} 
where $\mathcal{O}(U)$ is the ring of functions on $U$ acting on $\mathbb{C}_{p}$ by evaluation at $p$. If we choose local coordinates $x_{1}, \ldots, x_{n}$ at $p$, and choose vector fields $\partial_{1}, \ldots, \partial_{n}$ on $U$ such that the value of $\partial_{i}$ at $p$ is $\partial / \partial x_{i}$, then

$$
\delta_{p}(U) \simeq \mathbb{C}\left[\partial_{i}\right]_{i=1, \ldots, n} .
$$

The definition of the sheaf of $\delta$-functions supported at $p$ in the categories of twisted left and right $D$-modules is similar.

If we deal with a smooth stack ( $\operatorname{such}$ as $\operatorname{Bun}_{G}$ ) rather than a variety, then a point $\mathcal{P}$ may well come with a non-trivial group $\operatorname{Aut}(\mathcal{P})$ of automorphisms (in the case of a $G$-bundle $\mathcal{P}$, this is the group of global automorphisms of $\mathcal{P}$, which is a subgroup of $G$ ). In this case, instead of a single $\delta$-function $D$-module supported at $\mathcal{P}$, we have a category of $D$-modules supported at $\mathcal{P}$ which is equivalent to the category of representations of $\operatorname{Aut}(\mathcal{P})$. In this case, we denote by $\delta_{\mathcal{P}}$ the $D$-module corresponding to the regular representation of Aut $(\mathcal{P})$ (i.e. the space of functions on $\operatorname{Aut}(\mathcal{P})$ ). For further comments about these $D$-modules, see Remark 6.2 at the end of Section 6.5.

3.3. Quantum dualities. Supersymmetric quantum gauge theories in $4 d$ possess important dualities generalizing the electromagnetic duality. Each duality relates various attributes of two gauge theories, which usually have different coupling constants and often different groups, $G$ and its Langlands dual ${ }^{L} G$. Upon compactification of the $4 d$ theory on a Riemann surface $X$, these dualities give rise to interesting equivalences of categories, which can be viewed as quantum versions of the geometric Langlands correspondence. Tracing these equivalences to the "first principles" of $4 \mathrm{~d}$ gauge theory yields unexpected insights into the quantum geometric Langlands theory, which would be difficult to realize otherwise.

Gauge theory for a general compact Lie group can be decomposed into gauge theories for the simple and abelian factors, up to some topological subtleties which however do not affect the non-perturbative dynamics behind quantum duality symmetries.

We will discuss the properties of general gauge theories in Section 9. For now, we will focus on a connected simple Lie group $G$ over $\mathbb{C}$. Denote by $m$ the lacing number of $G$ - the maximal number of edges connecting two vertices of the Dynkin diagram of $G$. Thus, $m$ is 1 for simply-laced groups, and is equal to 2 or 3 for non-simply laced simple Lie groups.

There are two basic types of dualities: the orientation reversal symmetry $R$ and the dualities corresponding to elements of a subgroup of $P G L_{2}(\mathbb{Z})$, which depends on the bulk theory. We start by outlining their action on bulk theories $\mathcal{T}_{\kappa}^{G}$ introduced above.

- $R$ preserves the group $G$ but sends $\kappa$ to $-\kappa: \mathcal{T}_{\kappa}^{G} \mapsto \mathcal{T}_{-\kappa}^{G}$.

- For a given $G$, only a particular subgroup of $P G L_{2}(\mathbb{Z})$ acts by duality transformations. Furthermore, these transformations in general change not only the parameter $\kappa$ but the group $G$ as well, so in fact it is better to view them as forming a groupoid rather than a group.

Let us describe this groupoid, which we will denote by $\mathcal{G}_{\kappa}^{G}$.

The group $P G L_{2}(\mathbb{Z})$ naturally acts on the projective line, which gives rise to the standard action on $\kappa$ viewed as a point on the projective line with respect to a particular coordinate:

$$
\kappa \mapsto \frac{a \kappa+b}{c \kappa+d}
$$


Now introduce the following elements of $P G L_{2}(\mathbb{Z})$ :

$$
S_{m}=\left(\begin{array}{cc}
0 & -1 \\
m & 0
\end{array}\right) \quad \kappa \mapsto \check{\kappa}=-1 / m \kappa
$$

and

$$
T=\left(\begin{array}{ll}
1 & 1 \\
0 & 1
\end{array}\right) \quad \kappa \mapsto \kappa+1 .
$$

Denote by $n(G)$ the minimum positive coefficient of a well-defined Chern-Simons action for $G_{c}$ [17] (equivalently, the level of the affine Kac-Moody algebra corresponding to the minimal line bundle $\mathcal{L}_{G}$ on the moduli stack of $G$-bundles on a curve X) ${ }^{10}$ For example, $n(G)=1$ if $G$ is simply-connected simple Lie group. Likewise, we denote by $n\left({ }^{L} G\right)$ the corresponding number for ${ }^{L} G$. Let $\mathcal{S}_{G}$ (resp., $\mathcal{S}_{L_{G}}$ ) be the subgroup of $P S L_{2}(\mathbb{Z})$ generated by $T^{n(G)}$ and $S_{m} T^{n\left({ }^{L} G\right)} S_{m}$ (resp., $T^{n\left({ }^{L} G\right)}$ and $\left.S_{m} T^{n(G)} S_{m}\right)$.

We define the groupoid $\mathcal{G}_{\kappa}^{G}$ as the category with the objects (which we will sometimes refer to as nodes) labeled by pairs

$$
\left(G, \kappa^{\prime}\right), \quad \kappa^{\prime} \in \mathcal{S}_{G} \cdot \kappa, \quad \text { and } \quad\left({ }^{L} G, \kappa^{\prime}\right), \quad \kappa^{\prime} \in \mathcal{S}_{L_{G}} \cdot \check{\kappa},
$$

where $\check{\kappa}=-1 / m \kappa$. We have morphisms (arrows) from $\left(G, \kappa^{\prime}\right)$ to $\left(G, \kappa^{\prime \prime}\right)$ for each element $g$ in $\mathcal{S}_{G}$ such that $\kappa^{\prime \prime}=g\left(\kappa^{\prime}\right)$; from $\left(G, \kappa^{\prime}\right)$ to $\left({ }^{L} G, \kappa^{\prime \prime}\right)$ for each element $g$ in $\mathcal{S}_{L_{G}} S_{m}$ or $S_{m} \mathcal{S}_{G}$ such that $\kappa^{\prime \prime}=g\left(\kappa^{\prime}\right)$; and similarly for the morphisms starting from nodes labeled by ${ }^{L} G$.

Now we consider the action of these symmetries on the boundary conditions discussed above and the corresponding categories.

The symmetry $R$ sends the bulk theory $\mathcal{T}_{\kappa}^{G}$ with a boundary condition $B$ to the bulk theory $\mathcal{T}_{-\kappa}^{G}$ with a boundary condition that we denote by $R(B)$. The resulting ribbon category should be dual to the original one, so that we have an equivalence

$$
\mathrm{e}_{-\kappa}^{G}(R(B)) \simeq \mathcal{C}_{\kappa}^{G}(B)^{\vee}
$$

In general, we need to consider here the dual of the derived, of a DG, category. However, for irrational values of $\kappa$, it appears that it is sufficient to work with the abelian categories.

On the other hand, the action of the duality groupoid $\mathcal{G}_{\kappa}^{G}$ should take the ribbon categories to equivalent ones:

$$
\mathrm{e}_{g(\kappa)}^{g(G)}(g(B)) \simeq \mathrm{e}_{\kappa}^{G}(B)
$$

for every $g \in \mathcal{S}_{G}$ (in this case, $g(G)=G$ ) and $g \in S_{m} \cdot \mathcal{S}_{G}$ (in this case, $g(G)={ }^{L} G$ ).

3.4. Examples of the action of quantum dualities on boundary conditions. Now we look at how $R$ and the duality groupoid $\mathcal{G}_{\kappa}^{G}$ act on the basic boundary conditions.

\footnotetext{
${ }^{10}$ Here is the first place where spin subtleties occur: for some $G$, the minimal Chern-Simons action is only defined on spin manifolds unless we replace $n(G)$ by $2 n(G)$. Equivalently, for those groups we need to pick the square root of the canonical line bundle on $X$ in order to define the line bundle $\mathcal{L}_{G}$; without such a choice, we can only define $\mathcal{L}_{G}^{\otimes 2}$.
} 
(i) The action of $R$ preserves the Neumann, Dirichlet, and Nahm boundary conditions. In fact, for irrational $\kappa$ the equivalences (3.1) hold in these cases at the level of abelian categoies.

For example, for irrational $\kappa$ the dual category to $\mathrm{KL}_{\kappa}(G)$ is $\mathrm{KL}_{-\kappa}(G)$. This can be seen from the Kazhdan-Lusztig equivalence between the category $\operatorname{KL}_{\kappa}(G)$ with irrational $\kappa$ and the category $U_{q}(\mathfrak{g})$-mod of finite-dimensional $U_{q}$-modules, where $q=e^{\pi i / \kappa}$. As explained in Example 2.10.14 of [21], the dual category to the latter is equivalent to the opposite category, which is known to be equivalent to $U_{q^{-1}}(\mathfrak{g})$-mod and hence to $\mathrm{KL}_{-\kappa}(G)$ via the Kazhdan-Lusztig equivalence.

(ii) The action of $S_{m}$ exchanges the Neumann and principal Nahm boundary conditions. The corresponding categories are therefore expected to be equivalent:

$$
\mathrm{KL}_{\kappa}(G) \simeq \text { Whit }_{1 / m \kappa}\left({ }^{L} G\right) .
$$

This is essentially the statement of a conjecture of Gaitsgory and Lurie, proved by Gaitsgory for irrational $\kappa[44,46,11$

(iii) The $S_{m}$-dual of the Dirichlet boundary condition is much more complicated if $G$ is non-abelian (see [40, 41, 36, 37]). (If $G$ is abelian, there is no difference between the Dirichlet and Nahm boundary conditions, and therefore the dual of Dirichlet is Neumann.) From the perspective of boundary conditions in $4 \mathrm{~d}$ gauge theory, this is the reason why it is difficult to construct the quantum geometric Langlands correspondence. All we can say at the outset is that the corresponding category should be equivalent to the category of right $1 / m \kappa$-twisted $D$-modules on $\operatorname{Gr}_{L_{G}}$. Similar considerations apply to the intermediate Nahm boundary conditions.

(iv) The action of $T^{p}, p \in n(G) \cdot \mathbb{Z}$. If $B$ is any of the Nahm boundary conditions or a Dirichlet boundary condition, then $T^{p}(B)=B$, but for the Neumann boundary conditions, $T^{p}(B) \neq B$ for $p \in \mathbb{Z}$. In any case, we expect the equivalences

$$
\mathrm{C}_{\kappa+p}^{G}\left(T^{p}(B)\right) \simeq \mathrm{e}_{\kappa}^{G}(B)
$$

In other words,

$$
\mathcal{C}_{\kappa}^{G}\left(T^{p}(B)\right) \simeq \mathcal{C}_{\kappa-p}^{G}(B)
$$

In particular, the equivalences

$$
D_{\kappa}(\mathrm{Gr}) \simeq D_{\kappa+p}(\mathrm{Gr}), \quad \text { Whit }_{\kappa}(G) \simeq \text { Whit }_{\kappa+p}(G), \quad \forall p \in n(G) \cdot \mathbb{Z}
$$

are given by tensoring with the appropriate line bundle on $\mathrm{Gr}_{G}$.

In Section 4 we conjecture that for irrational $\kappa$ the groupoid $\mathcal{G}_{\kappa}^{G}$ acts by equivalences on the categories of twisted $D$-modules on $\operatorname{Bun}_{G}$ and $\operatorname{Bun}_{L_{G}}$ (the situation becomes more subtle for rational values of $\kappa$ ).

\footnotetext{
${ }^{11}$ This is an example of an equivalence that for some groups requires a choice of a spin structure (or a square root of the canonical line bundle), because the category $\mathrm{KL}_{\kappa}(G)$ does not require such a choice, but the category Whit $1 / m \kappa\left({ }^{L} G\right)$ does require it for some $G$.
} 
3.5. Nomenclature for boundary conditions. We will consider boundary conditions in bulk theories of type $\mathcal{T}_{\kappa}^{G}$ discussed above and therefore for now we will keep $G$ in our notation for boundary conditions. But since all boundary conditions we consider are defined uniformly for all values of $\kappa$, we will not keep $\kappa$. Hence we use the notation $B^{G}$. Starting from every a boundary condition $B^{G}$, we can generate a whole family of boundary conditions: $R\left(B^{G}\right)$ and $g\left(B^{G}\right)$ with $g \in \mathcal{S}_{G}$ and $g\left(B^{L} G\right)$ with $g \in S_{m} \mathcal{S}_{G}$ by the action of the duality groupoid. This suggests the following nomenclature (it is similar but not identical to the one adopted in [16]).

For a given bulk theory $\mathcal{T}_{\kappa}^{G}$ we will use the following notation:

(i) $N_{0,1}^{G}$ for the (principal) Nahm boundary condition;

(ii) $N_{1,0}^{G}$ for the Neumann boundary condition;

(iii) $D_{0,1}^{G}$ for the Dirichlet boundary condition;

(iv) $N_{0,1}^{G, \rho}$ for the $\rho$-Nahm boundary condition.

Next, we denote by $N_{g \circ(0,1)}^{G}$ and $N_{g \circ(1,0)}^{G}$ (modulo the identification $N_{a p, a q}^{G}=N_{p, q}^{G}$ ) the images of these boundary conditions under $g \in \mathcal{S}_{G}$, with $g$ acting on the pairs $(p, q)$ according to its standard action on the column vectors $\left(\begin{array}{l}q \\ p\end{array}\right)$ (note the switch of $p$ and $q$ ).

Thus, every element in $\mathcal{G}_{\kappa}^{G}$ sends

$$
N_{p, q}^{G} \mapsto N_{g \circ(p, q)}^{g(G)}
$$

where $g(G)$ is either $G$ or ${ }^{L} G$. At the same time, each $g$ acts on $\kappa$ in the standard way.

Further, for the orientation reversal $R$ we define

$$
R: N_{p, q}^{G} \mapsto N_{p,-q}^{G}
$$

In this case, $R\left(\mathcal{T}_{\kappa}^{G}\right)=\mathcal{T}_{-\kappa}^{G}$, and so $R(\kappa)=-\kappa$.

We can give a similar definition of the families $D_{p, q}^{G}$ and $N_{p, q}^{G, \rho}$ starting from Dirichlet or $\rho$-Nahm boundary conditions in the bulk gauge theories $\mathcal{T}_{\kappa}^{G}$ and $\mathcal{T}_{\kappa}^{L}$. For example, $D_{0,1}^{G}$ denotes the Dirichlet boundary condition in $\mathcal{T}_{\kappa}^{G}$, while $D_{1,0}^{G}$ denotes the $S_{m}$-image of the Dirichlet boundary condition $D_{0,1}^{L} G$ in $\mathcal{T}_{\kappa}^{L} G$. Also, $R$ maps $D_{p, q}^{G}$ to $D_{p,-q}^{G}$, etc.

Using $R$ together with the groupoid of duality transformations, we obtain various nontrivial identifications between boundary conditions $N_{p, q}^{G}$ (beyond $N_{a p, a q}^{G}=N_{p, q}^{G}$ ). Each of them is expected to give rise to an equivalence between the corresponding ribbon categories of line defects. Some of these equivalence are highly non-trivial. For instance, we have

$$
\left(\mathcal{T}_{-1 / m \kappa}^{L_{G}}, N_{0,1}^{L_{G}}\right)=\left(\mathcal{T}_{\kappa}^{G}, N_{1,0}^{G}\right), \quad\left(\mathcal{T}_{-1 / m \kappa}^{L_{G}}, N_{1,0}^{L_{G}}\right)=\left(\mathcal{T}_{\kappa}^{G}, N_{0,1}^{G}\right) .
$$

The corresponding equivalences of categories are given by formula $(3.2)$ and its analogue in which we reverse $G$ and ${ }^{L} G, \kappa$ and $1 / m \kappa$. We will encounter more equivalences of this nature below. 
3.6. Extended families of boundary conditions. With some care, the duality groupoid can be extended to a larger groupoid involving all transformations generated by $T$ and $S_{m}$. The nodes of the extended groupoid involve gauge theories with gauge algebra $\mathfrak{g}$ or ${ }^{L} \mathfrak{g}$ but gauge group which may differ from $G$ or ${ }^{L} G$. They are furthermore modified by certain "discrete theta angles" [8]. It is possible to define well-behaved analogues of Dirichlet, Neumann, Nahm boundary conditions in these generalized theories, but it requires some extra structures, which we will describe in section 9 .

As a consequence, one may accordingly extend the range of pairs $(p, q)$ for which the above families of boundary conditions are well-defined. The compactification functors should map the categories associated to these boundary conditions to the categories of $D$-modules on $\operatorname{Bun}_{G}$ twisted by appropriate gerbes.

3.7. Vertex algebras at a junction. As explained in Section 2.2, we can attach a vertex algebra to a junction of two boundary conditions $B_{1}$ and $B_{2}$ in a bulk theory $\mathcal{T}$, possibly with some extra data attached to the junction. We now focus on the case of the bulk theory $\mathcal{T}=\mathcal{T}_{\kappa}^{G}$, as above, and denote the corresponding junction vertex algebra $V\left(\mathcal{T}_{\kappa}^{G}, B_{1} \rightarrow B_{2}\right)$ by $V_{\kappa}^{G}\left(B_{1} \rightarrow B_{2}\right)$. Then we have the corresponding functor $F_{\mathcal{T}, B_{1} \rightarrow B_{2}}$, which we now denote by $F_{\kappa, B_{1} \rightarrow B_{2}}^{G}$. Thus,

$$
F_{\kappa, B_{1} \rightarrow B_{2}}^{G}: \mathcal{C}_{\kappa}^{G}\left(B_{1}\right)^{\vee} \otimes \mathcal{C}_{\kappa}^{G}\left(B_{2}\right) \rightarrow V_{\kappa}^{G}\left(B_{1} \rightarrow B_{2}\right)-\bmod .
$$

This functor should satisfy the following conditions:

First,

$$
F_{\kappa, B_{1} \rightarrow B_{2}}^{G}(I \otimes I)=V_{\kappa}^{G}\left(B_{1} \rightarrow B_{2}\right)
$$

where $I$ denotes the identity object.

Second, if we apply any duality symmetry $g \in \mathcal{G}_{\kappa}^{G}$ as defined in Section 3.3 to all data, then the vertex algebra should be unchanged:

$$
V_{\kappa}^{G}\left(B_{1} \rightarrow B_{2}\right) \simeq V_{g(\kappa)}^{g(G)}\left(g\left(B_{1}\right) \rightarrow g\left(B_{2}\right)\right),
$$

and we should have a commutative diagram

$$
\begin{aligned}
& \mathcal{C}_{\kappa}^{G}\left(B_{1}\right)^{\vee} \otimes \mathcal{C}_{\kappa}^{G}\left(B_{2}\right) \quad \stackrel{F_{\kappa, B_{1} \rightarrow B_{2}}^{G}}{\longrightarrow} \quad V_{\kappa}^{G}\left(B_{1} \rightarrow B_{2}\right)-\bmod \\
& \downarrow \downarrow \\
& \mathcal{C}_{g(\kappa)}^{g(G)}\left(g\left(B_{1}\right)\right)^{\vee} \otimes \mathcal{C}_{g(\kappa)}^{g(G)}\left(g\left(B_{2}\right)\right) \stackrel{F_{g(\kappa), g\left(B_{1}\right) \rightarrow g\left(B_{2}\right)}^{g(())}}{\longrightarrow} V_{g(\kappa)}^{g(G)}\left(g\left(B_{1}\right) \rightarrow g\left(B_{2}\right)\right)-\bmod
\end{aligned}
$$

Third, under the orientation reversal $R$, we should have an isomorphism of vertex algebras

$$
V_{\kappa}^{G}\left(B_{1} \rightarrow B_{2}\right) \simeq V_{-\kappa}^{G}\left(R\left(B_{2}\right) \rightarrow R\left(B_{1}\right)\right),
$$

compatible with the functors $F_{\kappa, B_{1} \rightarrow B_{2}}^{G}$ and $F_{-\kappa, R\left(B_{2}\right) \rightarrow R\left(B_{1}\right)}^{G}$ and the equivalences

$$
\mathcal{C}_{\kappa}^{G}\left(B_{i}\right)^{\vee} \simeq \mathcal{C}_{-\kappa}^{G}\left(R\left(B_{i}\right)\right)
$$

Let us consider some basic examples. More examples will be presented in later sections. 
(i) Let $B_{1}=D_{0,1}^{G}$ and $B_{2}=N_{1,0}^{G}$ be the Dirichlet and Neumann boundary conditions, respectively. There is a standard junction between them described in [16] such that $V_{\kappa}^{G}\left(B_{1} \rightarrow B_{2}\right)$ is the affine Kac-Moody vertex algebra $V_{\kappa}(\mathfrak{g})$.

In this case, we have

$$
\mathcal{C}_{\kappa}^{G}\left(D_{0,1}^{G}\right)^{\vee} \otimes \mathcal{C}_{\kappa}^{G}\left(N_{1,0}^{G}\right)=\left(D_{-\kappa}\left(\operatorname{Gr}_{G}\right)\right)^{\vee} \otimes \mathrm{KL}_{\kappa}(G) \simeq D_{\kappa}\left(\operatorname{Gr}_{G}\right) \otimes \mathrm{KL}_{\kappa}(G),
$$

and there is indeed a functor from the latter category to the category of $V_{\kappa}(\mathfrak{g})$ modules (i.e., $\widehat{\mathfrak{g}}$-modules of level $\kappa$ ). Namely, we view $\mathrm{KL}_{\kappa}(G)$ as a subcategory of $\widehat{\mathfrak{g}}_{\kappa}$-mod and use the functor

$$
\mathcal{F} \in D_{\kappa}\left(\mathrm{Gr}_{G}\right), M \in \mathrm{KL}_{\kappa}(G) \mapsto \mathcal{F} \star M,
$$

where $\star$ denotes the categorical convolution functor (see [11, 30]).

Beilinson has conjectured (see the Introduction of [10], especially Remark (ii)) that the category of $\widehat{\mathfrak{g}}$-modules of any level $\kappa$ is expected to "fiber" over the stack of flat ${ }^{L} G$-bundles on the punctured disc. This conjecture suggests that the essential image of $\mathcal{C}_{\kappa}^{G}\left(D_{0,1}^{G}\right)^{\vee} \otimes \mathcal{C}_{\kappa}^{G}\left(N_{1,0}^{G}\right)$ under the functor $F_{\kappa, D_{0,1}^{G} \rightarrow N_{1,0}^{G}}^{G}$ is the subcategory of $\widehat{\mathfrak{g}}$-modules of level $\kappa$ supported on the formal neighborhood of the trivial flat bundle.

(ii) Let $B_{1}=N_{0,1}^{G}$ and $B_{2}=N_{1,0}^{G}$ be the Nahm and Neumann boundary conditions, respectively. Then there is a standard junction between them described in [59, 39, 16] such that $V_{\kappa}^{G}\left(B_{1} \rightarrow B_{2}\right)$ is the $\mathcal{W}$-algebra $\mathcal{W}_{\kappa}(\mathfrak{g})$.

The corresponding functor

$$
F_{\kappa, N_{0,1}^{G} \rightarrow N_{1,0}^{G}}^{G}: \text { Whit }_{\kappa}(G) \otimes \mathrm{KL}_{\kappa}(G) \rightarrow \mathcal{W}_{\kappa}(\mathfrak{g})-\bmod
$$

is constructed as follows. Recall [22, 33] (see Ch. 15 of [28] for a survey) that the Drinfeld-Sokolov reduction functor $H_{\mathrm{DS}}^{\mathfrak{g}}$ is defined as the semi-infinite cohomology of the nilpotent Lie subalgebra $\mathfrak{n}_{+}((z))$ of $\widehat{\mathfrak{g}}$ twisted by a non-degenerate character $\psi$ of $\mathfrak{n}_{+}((z))$ which takes non-zero values on the $(-1)$ st Fourier coefficients of the generating currents. This functor, applied to $\mathrm{KL}_{\kappa}(G)$, defines the restriction of $F_{\kappa, N_{0,1}^{G} \rightarrow N_{1,0}^{G}}^{G}$ to $\left.I \otimes \mathrm{KL}_{\kappa}(G)\right)$, where $I=\Psi_{\kappa}^{x, 0}$.

For irrational $\kappa$, it follows from the results of Arakawa [3, 4] that the functor $H_{\text {DS }}^{\mathfrak{g}}$ is exact, and its essential image in the category $\mathcal{W}_{\kappa}(\mathfrak{g})$-mod is a semi-simple subcategory with the simple modules $M_{(\lambda, 0), \kappa}=H_{\mathrm{DS}}^{\mathfrak{g}}\left(\mathbb{V}_{\lambda, \kappa}\right)$. See also [63] for some general results about this functor.

In order to incorporate the category $\operatorname{Whit}_{\kappa}(G)$, observe that for any dominant integral coweight $\mu^{\vee}$ of $G$, we can twist the character $\psi$ by the element $\mu^{\vee}(z) \in$ $H((z))$. Let us denote the quantum Drinfeld-Sokolov reduction functor with respect to this twisted character by $H_{\mathrm{DS}, \mu^{\vee}}^{\mathfrak{g}}$. This functor has been previously studied in [30, 16].

For any object $M$ of $K L_{\kappa}(G)$, the functor $F_{\kappa, N_{0,1}^{G} \rightarrow N_{1,0}^{G}}^{G}$ sends the object $\Psi_{\kappa}^{x, \mu^{\vee}} \otimes M$ of Whit ${ }_{\kappa}(G) \otimes \mathrm{KL}_{\kappa}(G)$ to the $\mathcal{W}_{\kappa}(\mathfrak{g})$-module $H_{\mathrm{DS}, \mu^{\vee}}^{\mathfrak{g}}(M)$. This can be generalized 
to arbitrary objects of Whit $_{\kappa}(G)$. Thus, Whit ${ }_{\kappa}(G)$ can be viewed as the category that controls the data of the Drinfeld-Sokolov reduction.

It is natural to conjecture that for irrational $\kappa$ the essential image of $F_{\kappa, N_{0,1}^{G} \rightarrow N_{1,0}^{G}}^{G}$ is a semi-simple subcategory of $\mathcal{W}_{\kappa}(\mathfrak{g})$-mod with simple modules

$$
M_{\left(\lambda, \mu^{\vee}\right), \kappa}=H_{\mathrm{DS}, \mu^{\vee}}^{\mathfrak{g}}\left(\mathbb{V}_{\lambda, \kappa}\right)=F_{\kappa, N_{0,1}^{G} \rightarrow N_{1,0}^{G}}^{G}\left(\Psi_{\kappa}^{x, \mu^{\vee}} \otimes \mathbb{V}_{\lambda, \kappa}\right)
$$

According to our conventions, the duality $S_{m}$ acts as follows:

$$
S_{m}\left(N_{0,1}^{G}\right)=N_{1,0}^{L_{G}}, \quad S_{m}\left(N_{1,0}^{G}\right)=N_{0,1}^{L_{G}}
$$

Hence $R S_{m}$ should send the junction $N_{0,1}^{G} \rightarrow N_{1,0}^{G}$ to $N_{0,1}^{L_{G}} \rightarrow N_{1,0}^{L_{G}}$, leading to the Feigin-Frenkel duality

$$
\mathcal{W}_{\kappa}(\mathfrak{g}) \simeq \mathcal{W}_{1 / m \kappa}\left({ }_{\mathfrak{g}}\right)
$$

Moreover, it follows from the diagram $(3.9)$ that the subcategories of $\mathcal{W}_{\kappa}(\mathfrak{g})$-mod and $\mathcal{W}_{1 / m \kappa}\left(L_{\mathfrak{g}}\right)$-mod that are the essential images of the functors $F_{\kappa, N_{0,1}^{G} \rightarrow N_{1,0}^{G}}^{G}$ and

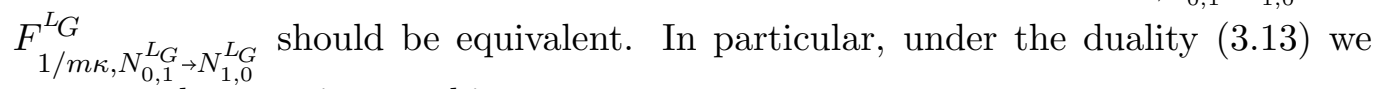
expect to have an isomorphism

$$
M_{\left(\lambda, \mu^{\vee}\right), \kappa}^{\mathfrak{g}} \simeq M_{\left(\mu^{\vee}, \lambda\right), 1 / m \kappa}^{L_{\mathfrak{g}}} .
$$

(see Conjecture 1.5 of [16]).12

Note that if this is the case, then the Whittaker category Whit ${ }_{\kappa}(G)$ can be realized for irrational $\kappa$ as a semi-simple subcategory $\mathcal{W}_{\kappa}(\mathfrak{g})_{m}$-mod of $\mathcal{W}_{\kappa}(\mathfrak{g})$-mod, which has as simple objects the modules

$$
M_{\left(0, \mu^{\vee}\right), \kappa}=H_{\mathrm{DS}, \mu^{\vee}}^{\mathfrak{g}}\left(\mathbb{V}_{0, \kappa}\right)
$$

with "magnetic" highest weights $\left(0, \mu^{\vee}\right), \mu^{\vee} \in{ }^{L} P^{+}$. The equivalence (3.2) between Whit $_{\kappa}(G)$ and $K L_{1 / m \kappa}\left({ }^{L} G\right)$ can therefore be rephrased as the statement that the category $\mathcal{W}_{\kappa}(\mathfrak{g})_{m}$-mod is equivalent, as a ribbon category, to $K L_{1 / m \kappa}\left({ }^{L} G\right)$. This statement (and its extension to negative rational $\kappa$ ) is essentially Conjecture 6.3 of [1].

(iii) More generally, if $B$ is any boundary condition, then we expect that the vertex algebra

$$
V_{\kappa}^{G}\left(D_{0,1}^{G} \rightarrow B\right)
$$

contains the affine vertex algebra $V_{\kappa^{\prime}}(\mathfrak{g})$, where $\kappa^{\prime}-\kappa \in n(G) \cdot \mathbb{Z}$, as a subalgebra. 13

\footnotetext{
12 This has been proved in $[5]$.

${ }^{13}$ This expectation is conditional on the existence of a physical junction $D_{0,1}^{G} \rightarrow B$ which preserves the global $G$ symmetry associated to the Dirichlet boundary condition. If such a junction exists, then by Noether's theorem we obtain the corresponding conserved currents which generate the affine vertex algebra at the junction.
} 
We cannot determine $\kappa^{\prime}$ on general grounds, because we can always modify the junction data $J_{12}$ by adding some holomorphic vertex algebra, and this operation may shift the level by a multiple of $n(G)$.

Furthermore, we expect that

$$
V_{\kappa}^{G}\left(N_{0,1}^{G} \rightarrow B\right)
$$

can be obtained by the quantum Drinfeld-Sokolov reduction of this affine subalgebra, and

$$
V_{\kappa}^{G}\left(N_{1, \kappa-\kappa^{\prime}}^{G} \rightarrow B\right)
$$

can be obtained by the BRST reduction of

$$
V_{\kappa}^{G}\left(D_{0,1}^{G} \rightarrow B\right) \otimes V_{-\kappa^{\prime}}(\mathfrak{g})
$$

(with respect to the diagonal action of $\widehat{\mathfrak{g}}$, with the level equal to twice the critical level). ${ }^{14}$

From the last two constructions, we can obtain many examples of vertex algebras, some of which we consider below.

These statements are consistent with the categorical statements: The averaging functor $D_{\kappa}\left(\mathrm{Gr}_{G}\right) \rightarrow$ Whit ${ }_{\kappa}(G)$ gives rise to a functor

$$
D_{\kappa}\left(\mathrm{Gr}_{G}\right) \otimes \mathrm{C}_{\kappa}^{G}(B) \rightarrow \text { Whit }_{\kappa}(G) \otimes \mathcal{C}_{\kappa}^{G}(B) .
$$

The composition of natural functors $D_{\kappa}\left(\mathrm{Gr}_{G}\right) \rightarrow D_{\kappa^{\prime}}\left(\mathrm{Gr}_{G}\right) \rightarrow \mathrm{KL}_{-\kappa^{\prime}}(G)$ (where the first functor is tensoring with a line bundle on $\mathrm{Gr}_{G}$ and the second functor is pulling back to $G((z))$ and taking $G[[z]]$-invariants with respect to the right action) and the equivalence $\mathrm{KL}_{-\kappa^{\prime}}(G)=\mathcal{C}_{-\kappa^{\prime}}^{G}\left(N_{1,0}^{G}\right) \simeq \mathcal{C}_{-\kappa}^{G}\left(N_{1, \kappa^{\prime}-\kappa}^{G}\right)$ gives a functor

$$
D_{\kappa}\left(\operatorname{Gr}_{G}\right) \otimes \mathcal{C}_{\kappa}^{G}(B) \rightarrow \mathcal{C}_{-\kappa}^{G}\left(N_{1, \kappa^{\prime}-\kappa}^{G}\right) \otimes \mathcal{C}_{\kappa}^{G}(B)
$$

(iv) Similar statements hold for general Nahm boundary conditions corresponding to an embedding $\rho: \mathfrak{s l}_{2} \rightarrow \mathfrak{g}$. In this case, the vertex algebra is the $\mathcal{W}$-algebra obtained by the generalized quantum Drinfeld-Sokolov reduction of $V_{\kappa}(\mathfrak{g})$ associated to the embedding $\rho$ [53].

3.8. Conformal blocks and compactification functors. It is natural to consider the spaces of conformal blocks and coinvariants of a junction vertex algebra $V_{\kappa}^{G}\left(B_{1} \rightarrow B_{2}\right)$.

Recall from Section 2.3 that we expect the coinvariants of $V_{\kappa}^{G}\left(B_{1} \rightarrow B_{2}\right)$ to be closely related to the Hom's of the images of line defects in $B_{1}$ and $B_{2}$ under the compactification functors.

More precisely, let $\mathcal{A}_{1} \in \mathrm{Ob}\left(\mathcal{C}_{\kappa}^{G}\left(B_{1}\right), \mathcal{A}_{2} \in \mathrm{Ob}\left(\mathcal{C}_{\kappa}^{G}\left(B_{2}\right)\right)\right.$. The compactification functors send them to twisted $D$-modules $\overline{\mathcal{A}}_{1}=F_{\kappa}^{G, B_{1}}\left(\mathcal{A}_{1}\right)$ and $\overline{\mathcal{A}}_{2}=F_{\kappa}^{G, B_{2}}\left(\mathcal{A}_{2}\right)$ on $\operatorname{Bun}_{G}$.

On the other hand, we have a $V_{\kappa}^{G}\left(B_{1} \rightarrow B_{2}\right)$-module $F_{\kappa, B_{1} \rightarrow B_{2}}^{G}\left(\mathcal{A}_{1}^{\vee} \otimes \mathcal{A}_{2}\right)$ which we will denote simply by $\mathcal{A}_{1}^{\vee} \otimes \mathcal{A}_{2}$. Then the statement of Conjecture 2.1 becomes

\footnotetext{
${ }^{14}$ These expectations are motivated from the existence of physical operations which convert a $D_{0,1}^{G}$ boundary condition to either $N_{0,1}^{G}$ or $N_{1, \kappa-\kappa^{\prime}}^{G}$. For example, an $N_{1, \kappa-\kappa^{\prime}}^{G}$ boundary condition can be obtained by "gauging the $G$ global symmetry of $D_{0,1}^{G}$ ". The effect of these operations on the junction vertex algebra is encoded by the above BRST reductions.
} 
Conjecture 3.1. Suppose that the functor $F_{\kappa, B_{1} \rightarrow B_{2}}^{G}$ is fully faithful. Then $\operatorname{Hom}\left(\overline{\mathcal{A}}_{1}, \overline{\mathcal{A}}_{2}\right)$ is isomorphic to the space of coinvariants of the $V_{\kappa}^{G}\left(B_{1} \rightarrow B_{2}\right)$-module $\mathcal{A}_{1}^{\vee} \otimes \mathcal{A}_{2}$.

An important special case arises when $B_{1}$ is the Dirichlet boundary condition $D_{0,1}^{G}$ whose global symmetry group is the group $G .15$ Then we can choose as $\overline{\mathcal{A}}_{1}$ the $\delta$-function $D$ module $\delta_{\mathcal{P}}$ supported at $\mathcal{P} \in \operatorname{Bun}_{G}$. Thus, given $\mathcal{A} \in \mathrm{Ob}\left(\mathcal{C}_{\kappa}^{G}(B)\right)$, we obtain a family of vector spaces $\operatorname{Hom}\left(\delta_{\mathcal{P}}, \overline{\mathcal{A}}\right)$. According to conjecture 3.1. they should be isomorphic to the spaces of coinvariants of the $V\left(B_{1} \rightarrow B_{2}\right)$-module corresponding to $\delta_{p} \otimes \mathcal{A}$.

We note that important special cases of this isomorphism were studied (in the language of branes) in [9] as an expression of a relation between the Kapustin-Witten and BeilinsonDrinfeld approaches to the Geometric Langlands correspondence (in this regard, see also Section 6.7).

In fact, as we explain in Section 6.5, we expect a stronger statement to be true. Indeed, on the one hand, $\operatorname{Hom}\left(\delta_{\mathcal{P}}, \overline{\mathcal{A}}\right)$ is a fiber of the $D$-module $\overline{\mathcal{A}}=F_{\kappa}^{G, B}(\mathcal{A})$ at $\mathcal{P} \in \operatorname{Bun}_{G}$. On the other hand, recall that we expect the vertex algebra $V_{\kappa}^{G}\left(D_{0,1}^{G} \rightarrow B\right)$ to contain an affine Kac-Moody vertex subalgebra $V_{\kappa^{\prime}}(\mathfrak{g})$ of level $\kappa^{\prime}$ such that $\kappa-\kappa^{\prime}=p \cdot n(G)$, where $p$ is an integer. This allows us to couple coinvariants of $V_{\kappa}^{G}\left(D_{0,1}^{G} \rightarrow B\right)$-modules to $G$-bundles on $X$. Mathematically, this means that for any $V_{\kappa}^{G}\left(D_{0,1}^{G} \rightarrow B\right)$-module, we can define its sheaf of coinvariants, which is a $\kappa^{\prime}$-twisted $D$-module on $\operatorname{Bun}_{G}$. This $D$-module can then be mapped to a $\kappa$-twisted $D$-module by tensoring it with the $p$ th power of the minimal line bundle $\mathcal{L}_{G}$, an infinite order generator of the Picard group of $\mathrm{Bun}_{G}$.

This enables us to define, under some natural assumptions, the compactification functor $F_{\kappa}^{G, B}$ in a mathematically rigorous way in terms of the localization functor for the vertex algebra $V_{\kappa}^{G}\left(D_{0,1}^{G} \rightarrow B_{2}\right)$, i.e. the functor assigning to a module over this vertex algebra its sheaf of coinvariants on $\operatorname{Bun}_{G}$ (see Section 6.5).

Another interesting case to consider is that of $B_{1}=N_{0,1}^{G}$ and $B_{2}=N_{1,0}^{G}$. Then the vertex algebra $V_{\kappa}^{G}\left(N_{0,1}^{G} \rightarrow N_{1,0}^{G}\right)$ is the $\mathcal{W}$-algebra $\mathcal{W}_{\kappa}(\mathfrak{g})$. If we take $\mathcal{A}_{1}=\Psi_{\kappa}^{x, \mu^{\vee}}$ and $\mathcal{A}_{2}=\mathbb{V}_{\lambda, \kappa}$, then the corresponding $\mathcal{W}_{\kappa}(\mathfrak{g})$-module is $M_{\left(\lambda, \mu^{\vee}\right), \kappa}$ which appeared in formula (3.12). Conjecture 3.1 then implies that there is an isomorphism between $\operatorname{Hom}\left(\Psi_{\kappa}^{x, \mu^{\vee}}, \mathcal{D}_{\kappa}^{x, \lambda}\right)$ and the space of coinvariants of the $\mathcal{W}_{\kappa}(\mathfrak{g})$-module $\left.M_{(\lambda, \mu \vee}\right)_{\kappa}$. In the case $\mu^{\vee}=0$ and $\lambda=0$, a closely related isomorphism (in the language of branes) was proposed in [59] as a possible interpretation of the AGT conjecture [2].

Remark 3.1. This statement has a multi-point generalization: an isomorphism between the space $\operatorname{Hom}\left(\Psi_{\kappa}^{x_{i}, \mu_{i}^{\vee}}, \mathcal{D}_{\kappa}^{x_{i}, \lambda_{i}}\right)$ and the space of coinvariants of the modules $M_{\left(\lambda_{i}, \mu_{i}^{\vee}\right), \kappa}$ inserted at the points $x_{i} \in X$. Consider the case $\mathfrak{g}=\mathfrak{s l}_{2}, X=\mathbb{P}^{1}$, and suppose that the set of points $x_{i}$ is a disjoint union of two subsets; for points of one of subset we have $\lambda_{i}=0$ and $\mu_{i}^{\vee}$ is the fundamental coweight, and for points of the other subset we have arbitrary $\lambda_{i}$ but $\mu_{i}^{\vee}=0$. Then, if we allow the points of the first kind to vary, we can view this isomorphism as a reformulation of the quantum separation of variables linking conformal blocks of $\widehat{\mathfrak{s l}}_{2}$

\footnotetext{
${ }^{15}$ More generally, the global symmetry group of the general Nahm pole boundary conditions $N_{1,0}^{G, \rho}$ is the centralizer of $\rho$.
} 
and the Virasoro algebra [66, 67, 32] (at the critical level, it becomes the separation of variables of the corresponding Gaudin model [23]). Thus, the above isomorphism (with points corresponding to dominant integral weights or to the fundamental coweights) could be viewed as a generalization of the quantum separation of variables to Lie algebras of higher rank. We leave the details to a future work.

Replacing $N_{1,0}^{G}$ by another boundary condition $B$, we obtain a generalization of the above isomorphism, which expresses coinvariants of modules over $V_{\kappa}^{G}\left(N_{0,1}^{G} \rightarrow B\right)$ as Hom's between the corresponding $D$-modules on $\operatorname{Bun}_{G}$.

Indeed, recall from item (iii) of Section 3.7 that if the junction $N_{0,1}^{G} \rightarrow B$ is obtained from a junction $D_{0,1}^{G} \rightarrow B$, then we expect the vertex algebra $V_{\kappa}^{G}\left(N_{0,1}^{G} \rightarrow B\right)$ to be the quantum Drinfeld-Sokolov reduction of $V_{\kappa}^{G}\left(D_{0,1}^{G} \rightarrow B\right)$. Now, given an object $\mathcal{A}$ of the category $\mathcal{C}_{\kappa}^{G}(B)$, we obtain a family of "magnetic" $V_{\kappa}^{G}\left(N_{0,1}^{G} \rightarrow B\right)$-modules $H_{\mathrm{DS}, \mu}^{\mathfrak{g}}(\mathcal{A})$. Applying Conjecture 3.1 we obtain that the space of coinvariants of the $V_{\kappa}^{G}\left(N_{0,1}^{G} \rightarrow B\right)$-module $H_{\mathrm{DS}, \mu^{\vee}}^{\mathfrak{g}}(\mathcal{A})$ is isomorphic to $\operatorname{Hom}\left(\Psi_{\kappa}^{x, \mu^{\vee}}, F_{\kappa}^{G, B}(\mathcal{A})\right)$.

\section{Quantum Langlands Dualities of Twisted $D$-Modules}

It is important to realize that using the quantum dualities, we can generalize the compactification functor to a whole family of functors from the category $\mathcal{C}_{\kappa}^{G}(B)$ to various categories of twisted $D$-modules on $\operatorname{Bun}_{G}$ and $\operatorname{Bun}_{L_{G}}$. This will naturally lead us to postulate the existence of certain functors between the categories $D_{\kappa}\left(\operatorname{Bun}_{G}\right)$. We will conjecture that for irrational $\kappa$ these give rise to a plethora equivalences of categories, generalizing the quantum geometric Langlands correspondence (1.1).

4.1. A family of dual compactification functors. Recall the duality groupoid $\mathcal{G}_{\kappa}^{G}$ introduced in Section 3.3. Let us observe that a duality does not affect the group of global symmetries of a boundary condition. In particular, for any $g \in \mathcal{G}_{\kappa}^{G}$, the boundary condition $D_{p, q}^{G}=g^{-1}\left(D_{0,1}^{g(G)}\right)$ should have the same global symmetry group as the Dirichlet boundary condition $D_{0,1}^{g(G)}$, which is the group $g(G)$. Therefore, arguing as above, for any junction $D_{p, q}^{G} \rightarrow B$ in the bulk theory $\mathcal{T}_{\kappa}^{G}$, where $D_{p, q}^{G}=g^{-1}\left(D_{0,1}^{g(G)}\right)$, we obtain a functor from the category $\mathrm{e}_{\kappa}^{G}(B)$ to the category of twisted $D$-modules on $\operatorname{Bun}_{g(G)}$.

To determine the twisting parameter, observe that the junction $D_{p, q}^{G} \rightarrow B$ in the bulk theory $\mathcal{T}_{\kappa}^{G}$ can be seen in another duality frame as the junction $D_{0,1}^{g(G)} \rightarrow g(B)$ in the bulk theory $\mathcal{T}_{g(\kappa)}^{g(G)}$, which gives rise to a functor (in general, of derived categories)

$$
F_{g(\kappa)}^{g(G), g(B)}: \mathcal{C}_{g(\kappa)}^{g(G)}(g(B)) \rightarrow D_{g(\kappa)}\left(\operatorname{Bun}_{g(G)}\right) .
$$

But the category $\mathcal{C}_{g(\kappa)}^{g(G)}(g(B))$ should be equivalent to $\mathcal{C}_{\kappa}^{G}(B)$, so $F_{g(\kappa)}^{g(G), g(B)}$ gives rise to a functor

$$
{ }_{g} F_{\kappa}^{G, B}: \mathcal{C}_{\kappa}^{G}(B) \rightarrow D_{g(\kappa)}\left(\operatorname{Bun}_{g(G)}\right) .
$$

The functors ${ }_{g} F_{\kappa}^{G, B}$ are generalizations of the compactification functor $F_{\kappa}^{G, B}$ (which corresponds to $g$ being the identity). They correspond to junctions $D_{p, q}^{G} \rightarrow B$ in the same sense 
in which the compactification functor $F_{\kappa}^{G, B}$ corresponds to a junction $D_{0,1}^{G} \rightarrow B$. Note that in both cases there could be multiple junctions between these boundary conditions, but the corresponding functors ${ }_{g} F_{\kappa}^{G, B}$ should be equivalent to each other according to Conjecture 2.2 (see Conjecture 6.1 for a more precise formulation).

Once the functors $F_{\kappa}^{G, B}$ labeled by $g$ in the groupoid $\mathcal{G}_{\kappa}^{G}$ have been defined, it is natural to suppose that there is also a collection of duality functors $\mathcal{E}_{\kappa}^{G, g}$, labeled by the same groupoid, acting between the corresponding categories of twisted $D$-modules on $\operatorname{Bun}_{G}$ and $\operatorname{Bun}_{L_{G}}$ and intertwining the functors ${ }_{g} F_{\kappa}^{G, B}$ for all boundary $B$ conditions. This leads to a vast generalization of the idea of quantum Geometric Langlands duality (1.1), which from the $4 \mathrm{~d}$ gauge theory point of view is just one of many duality functors; namely, the functor $\mathcal{E}_{\kappa}^{G, S_{m}}$ attached to the duality $S_{m}$.

Putting aside the subtleties related to the spin structures, which will be addressed in later sections, we expect that for irrational $\kappa$ these functors $\mathcal{E}_{\kappa}^{G, g}$ are in fact equivalences between categories of twisted $D$-modules on $\mathrm{Bun}_{G}$ and $\mathrm{Bun}_{L_{G}}$. In other words, we conjecture that for each $g \in \mathcal{G}_{\kappa}^{G}$ there is an equivalence $\mathcal{E}_{\kappa}^{G, g}$ between $D_{\kappa}\left(\operatorname{Bun}_{G}\right)$ and $D_{g(\kappa)}\left(\operatorname{Bun}_{g(G)}\right)$ which intertwines the equivalences $\mathcal{C}_{\kappa}^{G}(B) \simeq \mathcal{C}_{g(\kappa)}^{g(G)}(g(B))$ via the corresponding compactification functors, for all boundary conditions $B$.

Before stating the conjecture, it is useful to recall the subgroup $\mathcal{S}_{G}$ of $P G L_{2}(\mathbb{Z})$ generated by $T^{n(G)}$ and $S_{m} T^{n\left({ }^{L} G\right)} S_{m}$. Each element $g \in \mathcal{S}_{G}$ gives rise to a particular element (arrow) of the groupoid $\mathcal{G}_{\kappa}^{G}$ connecting $(G, \kappa)$ to $(G, g(\kappa))$. In this case, we write $g(G)=G$. Similarly, each element $g \in S_{m} \cdot \mathcal{S}_{G}$ gives rise to the element of $\mathcal{G}_{\kappa}^{G}$ connecting $(G, \kappa)$ to $\left({ }^{L} G, g(\kappa)\right)$. In case case, we write $g(G)={ }^{L} G$. Likewise, for $g \in \mathcal{S}_{L_{G}}$ and $g \in S_{m} \cdot \mathcal{S}_{L_{G}}$. When no confusion can arise, we use the same notation $g$ for the corresponding elements of the groupoid $\mathcal{G}_{\kappa}^{G}$.

Conjecture 4.1. Let $\kappa$ be irrational. Then for each $g \in \mathcal{G}_{\kappa}^{G}$, connecting the nodes $(G, \kappa)$ and $(g(G), g(\kappa))$, there is a functor

$$
\mathcal{E}_{\kappa}^{G, g}: D_{\kappa}\left(\operatorname{Bun}_{G}\right) \rightarrow D_{g(\kappa)}\left(\operatorname{Bun}_{g(G)}\right)
$$

that fits in a commutative diagram

$$
\begin{array}{ccc}
\mathcal{C}_{\kappa}^{G}(B) & \longrightarrow & \mathcal{C}_{g(\kappa)}^{g(G)}(g(B)) \\
F_{\kappa}^{G, B} \downarrow & \downarrow_{g(\kappa)}^{g(G), g(B)} \\
D_{\kappa}\left(\operatorname{Bun}_{G}\right) & \stackrel{\varepsilon_{\kappa}^{G, g}}{\longrightarrow} D_{g(\kappa)}\left(\operatorname{Bun}_{g(G)}\right)
\end{array}
$$

and these functors combine into a categorical action of the groupoid $\mathcal{G}_{\kappa}^{G}$ on the categories $D_{\kappa}\left(\operatorname{Bun}_{G}\right)$.

Furthermore, if $g=T^{n}, n=p \cdot n(G), p \cdot \mathbb{Z}$, then the functor

$$
\mathcal{E}_{\kappa}^{G, T^{n}}: D_{\kappa}\left(\operatorname{Bun}_{G}\right) \rightarrow D_{\kappa+n}\left(\operatorname{Bun}_{G}\right)
$$

is given by the formula

$$
\mathcal{F} \mapsto \mathcal{F} \otimes \mathcal{L}_{G}^{\otimes m},
$$

where $\mathcal{L}_{G}$ is a minimal line bundle on $\operatorname{Bun}_{G}$. In particular, the functor $\mathcal{E}_{\kappa}^{G, 1}$ is the identity functor. 
A categorical action of the groupoid $\mathcal{G}_{\kappa}^{G}$ means that for any pair $g, h \in \mathcal{G}_{\kappa}^{G}$, we have an isomorphism of functors

$$
i_{g, h}: \mathcal{E}_{h(\kappa)}^{h(G), g} \circ \mathcal{E}_{\kappa}^{G, h} \simeq \mathcal{E}_{\kappa}^{G, g h}
$$

and these isomorphisms satisfy a cocycle condition for every triple of elements of $\mathcal{G}_{\kappa}^{G}$ :

$$
i_{g, h k} \circ i_{h, k}=i_{g, h} \circ i_{g h, k} .
$$

This implies that the functors $\mathcal{E}_{\kappa}^{G, g}$ satisfy (in the categorical sense) the relations satisfied by the corresponding elements in the groupouid $\mathcal{G}_{\kappa}^{G}$ (all of these relations boil down to some relations in $P G L_{2}(\mathbb{Z})$ ). This leads to various non-trivial statements.

For example, Conjecture 4.1 states that $T^{n(G)} \in \mathcal{S}_{G}$ gives rise to an equivalence between the categories of twisted $\kappa$ - and $(\kappa+n(G))$-twisted $D$-modules on $\operatorname{Bun}_{G}$ obtained by tensoring a twisted $D$-module with the minimal line bundle $\mathcal{L}_{G}$ on $\operatorname{Bun}_{G}$, and likewise for $T^{n\left({ }^{(} G\right)}$ and $\operatorname{Bun}_{L_{G}}$. On the other hand, the duality transformation $S_{m} \in P G L_{2}(\mathbb{Z})$ should give rise to the equivalence (1.1):

$$
D_{\kappa}\left(\operatorname{Bun}_{G}\right) \simeq D_{-1 / m \kappa}\left(\operatorname{Bun}_{L_{G}}\right) .
$$

Therefore these equivalences should satisfy whatever relations are satisfied by $T^{n(G)}, T^{n\left({ }^{L} G\right)}$ and $S_{m}$ in the group $P G L_{2}(\mathbb{Z})$.

This is one of the reasons we find it more fruitful to look at the entire groupoid $\mathcal{G}_{\kappa}^{G}$ (rather than the specific equivalence $S_{m}$ ) as the collection of quantum Geometric Langlands dualities (qGL dualities for short). Another reason is that, as we show below, using the method of constructing vertex algebras via compositions of junctions, we can conjecturally construct the kernels of many of these qGL dualities directly. However, the vertex algebra corresponding to the duality $S_{m}$ may not be the optimal one (it may have unbounded conformal dimensions and other unfavorable features). Therefore, we may be better off constructing other qGL dualities first. Using these qGL dualities and the equivalences $T^{n(G)}$ and $T^{n\left({ }^{L} G\right)}$, we can then construct the qGL duality $S_{m}$ as well.

4.2. Kernels. Let us discuss these kernels in more detail. Let $g$ be an arrow between the nodes $(G, \kappa)$ and $(g(G), g(\kappa))$ in the groupoid $\mathcal{G}_{\kappa}^{G}$. Then, according to Conjecture 4.1 , there should be a functor

$$
\mathcal{E}_{\kappa, g(\kappa)}^{g(G), g}: D_{\kappa}\left(\operatorname{Bun}_{G}\right) \rightarrow D_{g(\kappa)}\left(\operatorname{Bun}_{g(G)}\right) .
$$

We hope to realize this functor as the correspondence induced by a kernel

$$
\mathcal{F}_{\kappa}^{G, g} \in D_{-\kappa, g(\kappa)}\left(\operatorname{Bun}_{G} \times \operatorname{Bun}_{g(G)}\right)
$$

which is obtained by applying a localization functor to the vertex algebra

$$
V_{g(\kappa)}^{g(G)}\left(D_{0,1}^{G} \rightarrow g\left(D_{0,1}^{G}\right)\right) .
$$

corresponding to a junction $D_{0,1}^{G} \rightarrow g\left(D_{0,1}^{G}\right)$.

Indeed, according to our general conjectures, this vertex algebra should have commuting actions of the affine Kac-Moody algebras $\widehat{\mathfrak{g}}$ of level $-\kappa$ and $\widehat{\mathfrak{g}^{\prime}}$ (where $\mathfrak{g}^{\prime}$ is the Lie algebra of $g(G)$ ) of level $g(\kappa)$. Therefore we can apply the localization functor with respect to both actions, which yields a twisted $D$-module in the category $D_{-\kappa, g(\kappa)}\left(\operatorname{Bun}_{G} \times \operatorname{Bun}_{g(G)}\right)$. 
According to our general conjectures, it should be independent of the junction data of $D_{0,1}^{G} \rightarrow g\left(D_{0,1}^{G}\right)$ and should coincide with the image of the identity object

$$
I \otimes I \in \mathcal{C}_{\kappa}^{G}\left(D_{0,1}^{G}\right)^{\vee} \otimes \mathcal{C}_{\kappa}^{g\left(D_{0,1}^{G}\right)}\left(g\left(D_{0,1}^{G}\right)\right)
$$

under the compactification functor of this category, viewed as a category arising in the $4 \mathrm{~d}$ gauge theory with the semisimple group $G \times g(G)$ and the coupling $(-\kappa, g(\kappa))$. In the next subsection we will explain a general strategy for constructing these kernels. We will give a number of examples in the later sections; in particular, two families of kernel vertex algebras in Sections 5 and 10.

The following heuristic point of view on the kernels $\mathcal{F}_{\kappa}^{G, g(G)}$ might be useful: let's think of the functors $F_{g(\kappa)}^{g(G), g(B)}$ as kind of bookkeeping devices, giving us "coordinate representations" of objects of the category $\mathcal{C}_{g(\kappa)(g(B)}^{g(G)} \simeq \mathcal{C}_{\kappa}^{G}(B)$. Indeed, given an object $A$ of $\mathcal{C}_{\kappa}^{G}(B)$, we obtain an object $g(A)$ of the category $\mathcal{C}_{g(\kappa)}^{g(G)}(g(B))$, and the functor $F_{\kappa}^{g(G), g(B)}$ then assigns to $g(A)$ a $g(\kappa)$-twisted $D$-module on $\operatorname{Bun}_{g(G)}$. Let's think of the collection of its fibers (to simplify notation, we denote $F_{\kappa}^{g(G), g(B)}(g(A))$ simply by $g(A)$ here)

$$
A \mapsto \operatorname{Hom}\left(\delta_{\mathcal{P}}, g(A)\right), \quad \mathcal{P} \in \operatorname{Bun}_{g(G)}
$$

as a categorical version of the set of " $g$-coordinates" of $A$, relative to a specific "basis" $\left\{\delta_{\mathcal{P}}\right\}_{\mathcal{P} \in \operatorname{Bun}_{g(G)}}$ in the category $D_{g(\kappa)}\left(\operatorname{Bun}_{g(G)}\right)$.

Now let's compare these " $g$-coordinates" to the "coordinates" of $A$ relative to the standard basis $\left\{\delta_{\mathcal{P}^{\prime}}\right\}_{\mathcal{P}^{\prime} \in \operatorname{Bun}_{G}}$ in the category $D_{\kappa}\left(\operatorname{Bun}_{G}\right)$ :

$$
A \mapsto \operatorname{Hom}\left(\delta_{\mathcal{P}^{\prime}}, A\right), \quad \mathcal{P}^{\prime} \in \operatorname{Bun}_{G} .
$$

As in linear algebra, converting one "coordinate representation" into another requires a categorical version of "change of basis matrix" which we can think of as a $D$-module on $\operatorname{Bun}_{G} \times \operatorname{Bun}_{g(G)}$ with fibers

$$
\operatorname{Hom}\left(\delta_{\mathcal{P}}, g\left(\delta_{\mathcal{P}^{\prime}}\right)\right), \quad\left(\mathcal{P}^{\prime}, \mathcal{P}\right),
$$

with the Hom's taken in the category $D_{g(\kappa)}\left(\operatorname{Bun}_{g(G)}\right.$.

Heuristically, for each $\mathcal{P} \in \operatorname{Bun}_{g(G)}$,

$$
\begin{aligned}
\operatorname{Hom}\left(\delta_{\mathcal{P}}, g(A)\right) & =\int_{\mathcal{P}^{\prime} \in \operatorname{Bun}_{G}} \operatorname{Hom}\left(\delta_{\mathcal{P}}, g\left(\delta_{\mathcal{P}^{\prime}}\right)\right) \otimes \operatorname{Hom}\left(g\left(\delta_{\mathcal{P}^{\prime}}\right), g(A)\right) \\
& =\int_{\mathcal{P}^{\prime} \in \operatorname{Bun}_{G}} \operatorname{Hom}\left(\delta_{\mathcal{P}}, g\left(\delta_{\mathcal{P}^{\prime}}\right)\right) \otimes \operatorname{Hom}\left(\delta_{\mathcal{P}^{\prime}}, A\right),
\end{aligned}
$$

where the first factor represents the kernel $\mathcal{F}_{\kappa}^{G, g}$.

The upshot of this discussion is that the kernel of the qGL duality $g$ should be a $D$-module on $\operatorname{Bun}_{G} \times \operatorname{Bun}_{g(G)}$ whose fibers at $\left(\mathcal{P}^{\prime}, \mathcal{P}\right)$ are $\operatorname{Hom}\left(\delta_{\mathcal{P}}, g\left(\delta_{\mathcal{P}^{\prime}}\right)\right)$. It should also be clear from the above discussion (see also Section 6.5 below) that this $D$-module can be obtained by applying the localization functor to the vertex algebra $V_{g(\kappa)}^{g(G)}\left(D_{0,1}^{G} \rightarrow g\left(D_{0,1}^{G}\right)\right)$. 
We expect that all of this works out nicely for irrational $\kappa$. For rational $\kappa$, some complications arise. We no longer expect that our functors yield equivalences between the corresponding categories of twisted $D$-modules. Rather, we expect such equivalences between certain "tempered" subcategories [6]. The reason is that from the $4 \mathrm{~d}$ gauge theory point of view [40, 41], the non-tempered part is accommodated by additional fields, denoted by $\sigma, \bar{\sigma}$ in [55], which become relevant at rational values of $\kappa$ as additional degrees of freedom [20]. They play a role similar to the role Arthur's $S L_{2}$ plays in the classical Langlands correspondence ([26], Sect. 6.2). See Remark 6.1] below for more details.

Another issue arising at rational $\kappa$ is that there are two derived categories of $\kappa$-twisted $D$-modules, dual to each other [18]. In order to extend our setup to rational $\kappa$ one should probably consider one of them for positive rational values of $\kappa$ and the other for negative ones 16

4.3. Extension of the compactification functor. The above construction is a special case of a general phenomenon: Whenever a boundary condition $B$ has a global symmetry group $H$, the compactification functor $F_{\kappa}^{G, B}$ can be extended to a functor

$$
F_{\kappa}^{G, B, H}: \mathcal{C}_{\kappa}^{G}(B) \rightarrow D_{\kappa,-\kappa_{H}}\left(\operatorname{Bun}_{G} \times \operatorname{Bun}_{H,(x)}\right) .
$$

Here $\operatorname{Bun}_{H,(x)}$ denotes the moduli stack of $H$-bundles on $X$ equipped with a trivialization on the formal disc around the point $x$. Such a trivialization is necessary in general in order to insert a line defect at $x$. However, specific line defects may only depend on a trivialization on the $n$th formal neighbourhood of $x$ for some integer $n \geq 0$, in which case the image of the functor can be well-defined on the corresponding quotient of $\operatorname{Bun}_{H,(x)}$ by a congruence subgroup of $H[[z]]$. It should be possible to express this statements as the requirement that the functor $F_{\kappa}^{G, B, H}$ should intertwine the appropriate actions of the loop group of $H$ on the source and target categories.

In particular, if we take the identify object $I$ in the category $\mathcal{C}_{\kappa}^{G}(B)$, then we do not insert any line defect at all, and therefore the corresponding twisted $D$-module $F_{\kappa}^{G, B, H}(I)$ is actually well-defined in $D_{\kappa,-\kappa_{H}}\left(\operatorname{Bun}_{G} \times \operatorname{Bun}_{H}\right)$. Thus, $F_{\kappa}^{G, B, H}(I)$ can be viewed as an extension of the compactification map, taking values in $D_{\kappa,-\kappa_{H}}\left(\operatorname{Bun}_{G} \times \operatorname{Bun}_{H}\right)$. We can obtain the functor $F_{\kappa}^{G, B, H}(I)$ by applying the localization functor to the vertex algebra $V_{\kappa}^{G}\left(D_{0,1}^{G} \rightarrow B\right)$ (for appropriate choices of junctions). Indeed, on general grounds we expect this vertex algebra to contain a Kac-Moody subalgebra $V_{\kappa^{\prime}}(\mathfrak{g}) \times V_{-\kappa_{H}^{\prime}}(\mathfrak{h})$, with $\kappa^{\prime}$ and $\kappa_{H}^{\prime}$ could in general differ from $\kappa$ by integral multiples of $n(G)$ and $n(H)$. Applying the localization functor to $V_{\kappa}^{G}\left(D_{0,1}^{G} \rightarrow B\right)$ with respect to this Kac-Moody subalgebra and tensoring the resulting $D$-modules by a line bundle if necessary, we obtain the desired $D$-module in $D_{\kappa, \kappa_{H}}\left(\operatorname{Bun}_{G} \times \operatorname{Bun}_{H}\right)$.

As an example, suppose that $B$ is also the Dirichlet boundary condition. Then $H=$ $G$. For irrational $\kappa$, the compactification map should produce the diagonal $D$-module in $D_{\kappa,-\kappa}\left(\operatorname{Bun}_{G} \times \operatorname{Bun}_{G}\right)$, and the compactification functor should be the natural functor

$$
D_{-\kappa}\left(\operatorname{Gr}_{G}\right) \rightarrow D_{-\kappa, \kappa}\left(\operatorname{Bun}_{G} \times \operatorname{Bun}_{G,(x)}\right)
$$

\footnotetext{
${ }^{16}$ We thank P. Yoo for useful comments on this issue.
} 
assigning to a compactly supported $D$-module on $\operatorname{Gr}_{G}$ a kernel on $\operatorname{Bun}_{G} \times \operatorname{Bun}_{G,(x)}$. This is a categorical version of a construction of the kernel of a convolution operator that is familiar from the theory of automorphic representations. The kernel of the convolution with a $G[[z]]$-invariant compactly supported object (a function or a $D$-module) on $\mathrm{Gr}_{G}$ is well-defined on $\operatorname{Bun}_{G} \times \operatorname{Bun}_{G}$, but the convolution with a general compactly supported object on $\operatorname{Gr}_{G}$ gives rise to a kernel on $\operatorname{Bun}_{G} \times \operatorname{Bun}_{G,(x)}$.

Now let $B$ be any duality image $g\left(D_{0,1}^{G}\right)$ of the Dirichlet boundary condition discussed above. Then we get an extended compactification functor

$$
D_{-g(\kappa)}\left(\operatorname{Gr}_{g(G)}\right) \rightarrow D_{-\kappa, g(\kappa)}\left(\operatorname{Bun}_{G} \times \operatorname{Bun}_{g(G),(x)}\right) .
$$

Applying it to the identity object of the category $D_{-g(\kappa)}\left(\mathrm{Gr}_{g(G)}\right)$, we obtain a $D$-module on $D_{-\kappa, g(\kappa)}\left(\operatorname{Bun}_{G} \times \operatorname{Bun}_{g(G)}\right)$ which is nothing but the kernel we discussed above. It can also be viewed as the qGL image of the diagonal $D$-module in $\operatorname{Bun}_{G} \times \operatorname{Bun}_{G}$ under the qGL duality $g$ applied along the second factor. Just as the categorical integral transform induced by the diagonal gives rise to the identity functor, integral transforms induced by these kernels give rise to functors taking twisted $D$-modules on $\operatorname{Bun}_{G}$ to their images on $\operatorname{Bun}_{g(G)}$ under the qGL duality $g$.

Thus, we see that junctions between $D_{0,1}^{G}$ and $g\left(D_{0,1}^{G}\right)$ are crucial for constructing qGL dualities. Namely, we expect the sheaves of coinvariants of the corresponding vertex algebras give rise to the qGL kernels. This begs the question: How to build junctions between $D_{0,1}^{G}$ and $g\left(D_{0,1}^{G}\right)$ ?

4.4. Building duality kernels. A particularly powerful method for constructing junctions between $D_{0,1}^{G}$ and its dual $g\left(D_{0,1}^{G}\right)$ is using compositions of junctions [16]. More generally, this method can allow us to produce many interesting junctions between boundary conditions which do not simultaneously admit weakly-coupled descriptions, such as $D_{0,1}^{G}$ and $D_{p, q}^{G}$.

In general, we will always compose junctions along boundary conditions of type $N_{p^{\prime}, q^{\prime}}^{G}$, whose category of boundary lines is semisimple for irrational $\kappa$. This considerably simplifies the construction of composite vertex algebras. We leave for future work calculations involving composition along other types of boundary conditions, as well as the limits of the objects we construct to rational values of $\kappa$.

Thus, our basic strategy is to "resolve" the junction between $D_{0,1}^{G}$ and $D_{p, q}^{G}$ by a composition of junctions of the form

$$
D_{p_{0}=0, q_{0}=1}^{G} \rightarrow N_{p_{1}, q_{1}}^{G} \rightarrow \cdots \rightarrow N_{p_{n-1}, q_{n-1}}^{G} \rightarrow D_{p_{n}=p, q_{n}=q}^{G}
$$

so that each the vertex algebra corresponding to each intermediate junction is a Kac-Moody or $\mathcal{W}$-algebra.

The resulting vertex algebra $V\left(\mathcal{T}, D_{0,1}^{G} \rightarrow D_{p, q}^{G}\right)$ is then an extension of an algebra which contains two Kac-Moody subalgebras at appropriate levels, together with a sequence of $\mathcal{W}$-algebras in-between. The extension involves diagonal objects in the products of two categories of the form $\mathrm{KL}_{\kappa_{i}}(G)$ or $\mathrm{KL}_{\kappa_{i}}\left({ }^{L} G\right)$ for appropriate intermediate levels.

As an important example, in which we don't have to deal with the subtleties related to the center and the spin structures, consider the case of the simple Lie group $G$ of type $E_{8}$, 
which is Langlands self-dual; it is simply-laced, simply-connected, and has trivial center. Then we can interpolate between $D_{0,1}^{G}$ and $D_{1,0}^{G}$ by $N_{1,-1}^{G}$.

- We have $T^{-1}\left(D_{0,1}^{G}\right)=D_{0,1}^{G}$ and $T^{-1}\left(N_{1,0}^{G}\right)=N_{1,-1}^{G}$. Therefore, if we apply $T^{-1}$ to the standard junction $D_{0,1}^{G} \rightarrow N_{1,0}^{G}$, we obtain the junction $D_{0,1}^{G} \rightarrow N_{1,-1}^{G}$, and according to formula (3.3), the latter supports $V_{\kappa+1}(\mathfrak{g})$.

- We have $R S\left(D_{1,0}^{G}\right)=D_{0,1}^{G}$ and $R S\left(N_{1,-1}^{G}\right)=N_{1,-1}^{G}$. Therefore, if we apply $R S$ to the junction $D_{0,1}^{G} \rightarrow N_{1,-1}^{G}$ (or equivalently, $R S T^{-1}$ to the standard junction $D_{0,1}^{G} \rightarrow N_{1,0}^{G}$ ), we obtain the junction $N_{1,-1}^{G} \rightarrow D_{1,0}^{G}$ (recall that the junction arrow is reversed under $R$, see formula (3.10)). The latter junction supports $V_{\kappa^{-1}+1}(\mathfrak{g})$, because if $g=R S T^{-1}$, then $g^{-1}(\kappa)=T S R(\kappa)=\kappa^{-1}+1$ (see formula (3.8)).

- The vertex algebra corresponding to the composite junction $D_{0,1}^{G} \rightarrow D_{1,0}^{G}$ is thus the following extension of $V_{\kappa+1}(\mathfrak{g}) \otimes V_{\kappa^{-1}+1}(\mathfrak{g})$ :

$$
V_{\kappa}^{G}\left(D_{0,1}^{G} \rightarrow D_{1,0}^{G}\right)=\bigoplus_{\lambda \in P^{+}(G)} \mathbb{V}_{\lambda, \kappa+1} \otimes \mathbb{V}_{\lambda, \kappa^{-1}+1}
$$

Conjecturally, if we apply the localization functor to this composite junction vertex algebra, we obtain a twisted $D$-module on $\operatorname{Bun}_{G} \times \operatorname{Bun}_{G}$ with the twists $\kappa+1$ along the first factor and $\kappa^{-1}+1$ along the second factor, which should be the kernel of the functor of qGL duality $\mathcal{E}_{-\kappa-1}^{G, T S T}$ corresponding to $T S T$ from $D_{-\kappa-1}\left(\operatorname{Bun}_{G}\right)$ to $D_{\kappa^{-1}+1}\left(\operatorname{Bun}_{G}\right)$. We will discuss a generalization of this junction vertex algebra, and the corresponding kernel, in Section 10.

As a more basic example, consider the composition of basic junctions from $D_{0,1}^{G}$ to $N_{1,0}^{G}$ and from $N_{1,0}^{G}$ to $D_{0,1}^{G}$. This composition gives the vertex algebra of (twisted) chiral differential operators on $G$, which has $V_{-\kappa}(\mathfrak{g}) \otimes V_{\kappa}(\mathfrak{g})$ as a vertex subalgebra [7]. For irrational $\kappa$, it is isomorphic to a direct sum of tensor products of the Weyl modules over $\widehat{\mathfrak{g}}$ of levels $-\kappa$ and $\kappa$ :

$$
V_{\kappa}^{G}\left(D_{0,1}^{G} \rightarrow D_{0,1}^{G}\right)=\bigoplus_{\lambda \in P^{+}(G)} \mathbb{V}_{\lambda,-\kappa} \otimes \mathbb{V}_{\lambda^{*}, \kappa},
$$

where $\lambda^{*}=-w_{0}(\lambda)$ is the highest weight of the irreducible $\mathfrak{g}$-module dual to the irreducible finite-dimensional $\mathfrak{g}$-module with highest weight $\lambda$. For irrational $\kappa$, we expect that the localization of this vertex algebra is the push-forward of the diagonal in $D_{-\kappa, \kappa}\left(\operatorname{Bun}_{G} \times \operatorname{Bun}_{G}\right)$. Thus, for irrational $\kappa, V_{\kappa}^{G}\left(D_{0,1}^{G} \rightarrow D_{0,1}^{G}\right)$ is the kernel of the identity functor $D_{\kappa}\left(\operatorname{Bun}_{G}\right) \rightarrow D_{\kappa}\left(\operatorname{Bun}_{G}\right)$, the qGL duality $g=1$.

\section{A family of KeRnel Vertex Algebras}

In this section we construct a family of junction vertex algebras $X_{p, q}(G)$ which we expect to give rise to kernels of specific quantum Langlands duality functors. The vertex algebra $X_{p, q}(G)$ is associated to a simple Lie group $G$ and two integers

$$
p \in n(G) \cdot \mathbb{Z}, \quad q \in n\left({ }^{L} G\right) \cdot \mathbb{Z},
$$

and it is equipped with the action of the affine Kac-Moody algebras $\widehat{\mathfrak{g}}_{-\kappa}$ and $\widehat{L}_{\mathfrak{g}_{g(\kappa)}}$, where

$$
g=\widetilde{T}^{p} S_{m} \widetilde{T}^{q}
$$


and

$$
\widetilde{T}=S_{m} T^{-1} S_{m}=\left(\begin{array}{cc}
1 & 0 \\
m & 1
\end{array}\right)
$$

When we apply the localization functor to $X_{p, q}(G)$, we obtain a twisted $D$-module

$$
\Delta_{-\kappa, g(\kappa)}\left(X_{p, q}(G)\right)
$$

on $\operatorname{Bun}_{G} \times \operatorname{Bun}_{L_{G}}$, with twists $-\kappa$ and $g(\kappa)$ along the first and the second factor, respectively We conjecture that $\Delta_{-\kappa, g(\kappa)}\left(X_{p, q}(G)\right)$ is a kernel of the qGL functor corresponding to $g=$ $\widetilde{T}^{p} S \widetilde{T}^{q}$ :

$$
\varepsilon_{\kappa}^{G, g}: D_{\kappa}\left(\operatorname{Bun}_{G}\right) \rightarrow D_{g(\kappa)}\left(\operatorname{Bun}_{L_{G}}\right)
$$

(see formula (6.3); note that $g(G)={ }^{L} G$ in this case).

The standard qGL duality, $g=S_{m}$, corresponds to the case $p=q=0$. The corresponding junction vertex algebra $X_{0,0}(G)$, obtained from the chain of junctions $D_{0,1} \rightarrow N_{1,0} \rightarrow$ $N_{0,1} \rightarrow D_{1,0}$, appears to coincide (for irrational $\kappa$ ) with the "master vertex algebra" recently proposed by Gaitsgory [47] as a candidate for a vertex algebra that could be used to construct a kernel of the qGL duality $S_{m}$ (this vertex algebra was also proposed earlier by Feigin; as far as we know, he has not published anything about it).

However, the vertex algebras $X_{0,0}(G)$ with $p \leq 0$ or $q \leq 0$ have conformal dimensions unbounded from below. This makes it more difficult to study these vertex algebras and to apply the localization functor to them (we likely need to take into account higher derived functors in this case and perhaps apply some regularization procedure, since the dimensions of the homogeneous components are infinite). However, we will show that in the case of positive $p$ and $q$, all conformal dimensions of $X_{p, q}(G)$ are non-negative; and furthermore, if $p>1$ and $q>0$ or $p>0$ and $q>1$, then only the vacuum vector has conformal dimension 0 and conformal dimensions of all other fields are strictly positive. Furthermore, the dimensions of all homogeneous components of fields corresponding to a fixed conformal dimension are then automatically finite.

In this sense, the kernel for the duality

$$
g=\widetilde{T}^{p} S_{m} \widetilde{T}^{q}=S_{m} T^{-p} S_{m} T^{-q} S_{m}
$$

with, say, $p \geq 1$ and $q>1$ has an advantage over the kernel for the standard qGL duality $S_{m}$. And once it is constructed, it can be used to construct the kernel for $S_{m}$.

For example, if $G$ is a simply-laced, simply connected group, then we have $m=1$ and we can take $p=1$, so using the relation $(S T)^{3}=1$, we can rewrite $g$ as $S T^{-1} S T^{-q} S=$ $T S T^{1-q} S$. If $n\left({ }^{L} G\right) \leq 2$, we can further take $q=2$, to obtain $T S T^{1-q} S=T^{2} S T$. But the action of $T$ and $T^{n\left({ }^{L} G\right)}$ corresponds (up to the spin and $\theta$-angle subtleties) to tensoring with a line bundle on $\operatorname{Bun}_{G}$ and $\operatorname{Bun}_{L_{G}}$, respectively. Therefore, once we construct a kernel for the duality $g$, we can construct a kernel for $S=S_{1}$ as well.

5.1. The case of $G=S L_{2}, p=q=0$. We first construct the kernel for the duality $S=S_{1}$ for $G=S L_{2}$ (i.e. $p=q=0$ ). For that we take the composition of the following chain of junctions:

in the bulk theory $\mathcal{T}_{-1 / \kappa}^{P S L_{2}}$.

$$
D_{0,1} \rightarrow N_{1,0} \rightarrow N_{0,1} \rightarrow D_{1,0}
$$


The vertex algebra corresponding to the first junction is $\widehat{\mathfrak{s l}}_{2}$ of level $-1 / \kappa$. The one corresponding to the second junction is $\mathcal{W}_{1 / \kappa}\left(\mathfrak{s l}_{2}\right) \simeq \mathcal{W}_{\kappa}\left(\mathfrak{s l}_{2}\right)$, i.e. the Virasoro algebra with the central charge

$$
c(\kappa)=13-6 \kappa-6 \kappa^{-1},
$$

because $N_{1,0} \rightarrow N_{0,1}=R\left(N_{0,1} \rightarrow N_{1,0}\right)$ and $-1 / \kappa=R(1 / \kappa)$. The one corresponding to the third junction,

$$
V_{-1 / \kappa}^{P S L_{2}}\left(N_{0,1} \rightarrow D_{1,0}\right) \simeq V_{-\kappa}^{S L_{2}}\left(D_{0,1} \rightarrow N_{1,0}\right),
$$

which is $\widehat{\mathfrak{s l}}_{2}$ of level $-\kappa$, because

$$
N_{0,1}^{P S L_{2}} \rightarrow D_{1,0}^{P S L_{2}}=S R\left(D_{0,1}^{S L_{2}} \rightarrow N_{1,0}^{S L_{2}}\right)
$$

and $-1 / \kappa=S R(-\kappa)$ (see formula (3.8)). Recalling the procedure of Section 2.5 for constructing compositions of junction vertex algebras, we obtain that for irrational $\kappa$ this vertex algebra is

$$
X_{0,0}\left(S L_{2}\right)=\bigoplus_{r \geq, s \geq 0} \mathbb{V}_{r,-\kappa} \otimes M_{(r, 2 s), \kappa} \otimes \mathbb{V}_{2 s,-1 / \kappa}
$$

where $\mathbb{V}_{m, \kappa}$ denotes the Weyl module over $\widehat{\mathfrak{s l}}_{2}$ with highest weight $m$ and (shifted) level $\kappa$, and $M_{(m, n), \kappa}$ denotes the irreducible highest weight module over the Virasoro algebra with the above central charge $c(\kappa)$ and conformal dimension

$$
h_{(m, n), \kappa}=\frac{1}{4}\left(m(m+2) \kappa^{-1}+n(n+2) \kappa\right)-\frac{1}{2}(m n+m+n) .
$$

Thus, the conformal dimension of the highest weight vector in $\mathbb{V}_{r,-\kappa} \otimes M_{(r, 2 s),-\kappa} \otimes \mathbb{V}_{2 s,-1 / \kappa}$ is

$$
h_{(r, 2 s), \kappa}-\frac{1}{4} r(r+2) \kappa^{-1}-\frac{1}{4} 2 s(2 s+2) \kappa=-\frac{1}{2}(2 r s+r+2 s) .
$$

We see this vertex algebra has fields with conformal dimensions unbounded from below, which is a drawback.

However, if we apply the localization functor to $X_{0,0}\left(S L_{2}\right)$ (perhaps, in the derived sense), we obtain a $D$-module on $\operatorname{Bun}_{S L_{2}} \times \operatorname{Bun}_{P S L_{2}}$ with the twisting $-\kappa$ along the first factor and $-1 / \kappa$ along the second factor. According to our general conjecture, it should be a kernel of the qGL functor corresponding to the duality transformation $S$ :

$$
\varepsilon_{\kappa}^{S L_{2}, S}: D_{\kappa}\left(\operatorname{Bun}_{S L_{2}}\right) \rightarrow D_{-1 / \kappa}\left(\operatorname{Bun}_{P S L_{2}}\right) .
$$

5.2. The case of $G=S L_{2}$, general $p, q$. We now generalize this to the duality transformations $g=\widetilde{T}^{p} S \widetilde{T}^{q}$ with arbitrary integers $p$ and $q$. Consider the following chain of junctions:

$$
D_{-p, 1} \rightarrow N_{1,0} \rightarrow N_{0,1} \rightarrow D_{1,-q}
$$

in the bulk theory $\mathcal{T}_{-1 / \kappa}^{P S L_{2}}$.

The vertex algebra corresponding to the first junction in (5.1) is $\widehat{\mathfrak{s l}}_{2}$ of level $1 /(p-\kappa)=$ $\widetilde{T}^{p}(-1 / \kappa)$, because $D_{-p, 1} \rightarrow N_{1,0}=\widetilde{T}^{-p}\left(D_{0,1} \rightarrow N_{1,0}\right)$. The vertex algebra corresponding to the second junction is $\mathcal{W}_{1 / \kappa}\left(\mathfrak{s l}_{2}\right) \simeq \mathcal{W}_{\kappa}\left(\mathfrak{s l}_{2}\right)$, the Virasoro algebra with the central charge $c(\kappa)$ (for the same reason as explained above). The one corresponding to the third junction,

$$
V_{-1 / \kappa}^{P S L_{2}}\left(N_{0,1} \rightarrow D_{1,-q}\right) \simeq V_{\kappa /(q \kappa-1)}^{S L_{2}}\left(D_{0,1} \rightarrow N_{1,0}\right),
$$


which is $\widehat{\mathfrak{s l}}_{2}$ of level $\kappa /(q \kappa-1)$, because

$$
N_{0,1}^{P S L_{2}} \rightarrow D_{1,-q}^{P S L_{2}}=S \widetilde{T}^{q} R\left(D_{0,1}^{S L_{2}} \rightarrow N_{1,0}^{S L_{2}}\right)
$$

and $-1 / \kappa=S \widetilde{T}^{q} R(\kappa /(q \kappa-1)$ ) (see formula (3.8)). Note that $q$ has to be even, because $\widetilde{T}^{q}$ is a legitimate duality in the bulk theory corresponding to $S L_{2}$, or equivalently, $T^{q}$ is a legitimate duality in the theory corresponding to $P S L_{2}$ (ignoring $\theta$-angle subtleties), only if $q$ is even (indeed, $n\left(P S L_{2}\right)=2$ ).

In the same way as above, we obtain that the corresponding vertex algebra is

$$
X_{p, q}\left(S L_{2}\right)=\bigoplus_{r, s \geq 0} \mathbb{V}_{r, \kappa /(q \kappa-1)} \otimes M_{(r, 2 s), \kappa} \otimes \mathbb{V}_{2 s, 1 /(p-\kappa)}
$$

The conformal dimension of the highest weight vector in $\mathbb{V}_{r, \kappa /(q \kappa-1)} \otimes M_{(r, 2 s), \kappa} \otimes \mathbb{V}_{2 s, 1 /(p-\kappa)}$ is

$$
\begin{aligned}
h_{(r, 2 s), \kappa}+\frac{1}{4} r(r+2) \frac{q \kappa-1}{\kappa}+\frac{1}{4} 2 s(2 s & +2)(p-\kappa) \\
& =\frac{1}{4}(r-2 s)^{2}+\frac{1}{4} r(r+2)(q-1)+s(s+1)(p-1)
\end{aligned}
$$

(note that this is always an integer if $q$ is even). Since $r, s \geq 0$, conformal dimensions are non-negative if $p, q \geq 1$, and are strictly positive, with the exception of the vacuum vector, if in addition either $p$ or $q$ are positive. But since $q$ has to be even, this is equivalent to $p \geq 1$ and $q \geq 2$.

Applying the localization functor to $X_{p, q}\left(S L_{2}\right)$, we obtain a twisted $D$-module on the product $\operatorname{Bun}_{S L_{2}} \times \operatorname{Bun}_{P S L_{2}}$. The twisting is $\kappa /(q \kappa-1)$ along the first factor and $1 /(p-\kappa)$ along the second factor. This should be a kernel of the qGL functor corresponding to the duality transformation $\widetilde{T}^{p} S \widetilde{T}^{q}$ :

$$
\mathcal{E}_{\kappa /(1-q \kappa)}^{S L_{2}, \widetilde{T}^{p} S \widetilde{T}^{q}}: D_{\kappa /(1-q \kappa)}\left(\operatorname{Bun}_{S L_{2}}\right) \rightarrow D_{1 /(p-\kappa)}\left(\operatorname{Bun}_{P S L_{2}}\right) .
$$

Note that the junction $D_{-p, 1} \rightarrow D_{1,-q}$ given by the composition (5.1) can be transformed by the duality $\widetilde{T}^{p}$ to $D_{0,1} \rightarrow D_{1-p q,-q}=D_{g(0,1)}$, where $g=\widetilde{T}^{p} S \widetilde{T}^{q}$. Therefore constructing the junction vertex algebra for $D_{-p, 1} \rightarrow D_{1,-q}$ is equivalent to constructing a junction vertex algebra for $D_{0,1} \rightarrow D_{g(0,1)}$. This is why we have used the same notation $X_{p, q}\left(S L_{2}\right)$ for this vertex algebra as we used at the beginning of this section. And in fact, if we make a change of variables $\kappa \mapsto \kappa /(q \kappa+1)$ in the above formulas, we obtain the kernel of

$$
\mathcal{E}_{\kappa}^{S L_{2}, g}: D_{\kappa}\left(\operatorname{Bun}_{S L_{2}}\right) \rightarrow D_{g(\kappa)}\left(\operatorname{Bun}_{P S L_{2}}\right),
$$

where $g=\widetilde{T}^{p} S \widetilde{T}^{q}$.

5.3. General case. For a general simple Lie group $G$ with lacing number $m$, we consider the composition of junctions

$$
D_{-p m, 1} \rightarrow N_{1,0} \rightarrow N_{0,1} \rightarrow D_{1,-q}
$$

in the bulk theory $\mathcal{T}_{-1 / m \kappa}^{L}$, where $p \in n(G) \mathbb{Z}, q \in n\left({ }^{L} G\right) \mathbb{Z}$. 
The vertex algebra corresponding to the first junction is $\widehat{L_{\mathfrak{g}}}$ of level $1 / m(p-\kappa)=$ $\widetilde{T}^{p}(-1 / m \kappa)$, because $D_{-m p, 1} \rightarrow N_{1,0}=\widetilde{T}^{-p}\left(D_{0,1} \rightarrow N_{1,0}\right)$. The vertex algebra corresponding to the second junction $N_{1,0} \rightarrow N_{0,1}=R\left(N_{0,1} \rightarrow N_{1,0}\right)$ is $\mathcal{W}_{1 / \kappa}\left({ }_{\mathfrak{g}}\right) \simeq \mathcal{W}_{\kappa}(\mathfrak{g})$. The vertex algebra corresponding to the third junction,

$$
V_{-1 / m \kappa}^{L_{G}}\left(N_{0,1} \rightarrow D_{1,-q}\right) \simeq V_{\kappa /(m q \kappa-1)}^{G}\left(D_{0,1} \rightarrow N_{1,0}\right),
$$

is $\widehat{\mathfrak{g}}$ of level $\kappa /(m q \kappa-1)$.

In the same way as before, we obtain that for irrational $\kappa$ the corresponding vertex algebra is

$$
X_{p, q}(G)=\bigoplus_{\lambda \in P^{+}, \mu^{\vee} \in P^{+}} \mathbb{V}_{\lambda^{*}, \kappa /(m q \kappa-1)} \otimes M_{\left(\lambda, \mu^{\vee}\right), \kappa} \otimes \mathbb{V}_{\mu^{\vee *}, 1 / m(p-\kappa)} .
$$

Here $\mathbb{V}_{\lambda^{*}, \kappa}$ denotes the Weyl module over $\widehat{\mathfrak{g}}$ generated from the irreducible finite-dimensional representation $\left(V_{\lambda}\right)^{*}$ of $\mathfrak{g}$ which is dual to $V_{\lambda}$. Since $\left(V_{\lambda}\right)^{*} \simeq V_{-w_{0}(\lambda)}$, we find that $\lambda^{*}=$ $-w_{0}(\lambda)$. The weight $\mu^{\vee *}$ of $L_{\mathfrak{g}}$ is defined similarly.

Recall from formula 3.12 that we denote by $M_{\left(\lambda, \mu^{\vee}\right), \kappa}(\mathfrak{g})$ the $\mathcal{W}_{\kappa}(\mathfrak{g})$-module

$$
M_{\left(\lambda, \mu^{\vee}\right), \kappa}(\mathfrak{g})=H_{\mathrm{DS}, \mu} \vee\left(\mathbb{V}_{\lambda, \kappa}\right) .
$$

The conformal dimension of its highest weight vector is (see [22])

$$
h_{\left(\lambda, \mu^{\vee}\right), \kappa}=\frac{1}{2 \kappa}(\lambda, \lambda+2 \rho)+\frac{\kappa}{2}\left(\mu^{\vee}, \mu^{\vee}+2 \rho^{\vee}\right)-\left\langle\lambda, \mu^{\vee}\right\rangle-\left\langle\rho, \mu^{\vee}\right\rangle-\left\langle\lambda, \rho^{\vee}\right\rangle .
$$

We use the invariant inner product $(\cdot, \cdot)_{\mathfrak{h}^{*}}$ on $\mathfrak{h}^{*}=P \underset{\mathbb{Z}}{\otimes} \mathbb{C}$ normalized so that the square length of the maximal root of $\mathfrak{g}$ is equal to 2 . This inner product enables us to identify $\mathfrak{h}^{*}$ with $\mathfrak{h}=\left({ }^{L} \mathfrak{h}\right)^{*}={ }^{L} P \underset{\mathbb{Z}}{\otimes} \mathbb{C}$. We denote the image of $\mu^{\vee} \in{ }^{L} P \subset \mathfrak{h}$ in $\mathfrak{h}^{*}$ under this identification by the same symbol. In particular, the $i$ th simple root of $L_{\mathfrak{g}}$ is identified with $2 \alpha_{i} /\left(\alpha_{i}, \alpha_{i}\right) \in \mathfrak{h}^{*}$.

In formula (5.3), $\left(\mu^{\vee}, \mu^{\vee}\right)$ stands for the square length $\left(\mu^{\vee}, \mu^{\vee}\right)_{\mathfrak{h}^{*}}$ of $\mu^{\vee} \in \mathfrak{h}^{*}$. Note that it is also equal to $m$ times the square length $\left(\mu^{\vee}, \mu^{\vee}\right)_{\left(L_{\mathfrak{h}}\right)^{*}}$ of this element with respect to the invariant inner product on $(\cdot, \cdot)_{\left(L_{\mathfrak{h}}\right)^{*}}$ normalized so that the square length of the maximal root of ${ }^{L} \mathfrak{g}$ is equal to 2 . That's why formula (5.3) stays invariant if we exchange $\mathfrak{g}$ and ${ }^{L_{\mathfrak{g}}}$ and replace $\kappa$ by $1 / m \kappa$ (and not $1 / \kappa$ ).

We also use the canonical pairing

$$
\langle\cdot, \cdot\rangle: \quad P \times{ }^{L} P \rightarrow \mathbb{Z}
$$

and the standard notation $\rho$ and $\rho^{\vee}$ for the elements of $P$ and ${ }^{L} P$, respectively, such that $\left\langle\rho, \alpha_{i}^{\vee}\right\rangle=1$ and $\left\langle\alpha_{i}, \rho^{\vee}\right\rangle=1$ for all $i$.

Conformal dimensions of the highest weight vectors of $\mathbb{V}_{\lambda^{*}, \kappa /(m q \kappa-1)}$ and $\mathbb{V}_{\mu^{\vee *}, 1 / m(p-\kappa)}$ coincide with those of $\mathbb{V}_{\lambda, \kappa /(m q \kappa-1)}$ and $\mathbb{V}_{\mu^{\vee}, 1 / m(p-\kappa)}$, respectively, and are equal to

$$
\frac{m q \kappa-1}{2 \kappa}(\lambda, \lambda+2 \rho)=\left(-\frac{1}{2 \kappa}+\frac{m q}{2}\right)(\lambda, \lambda+2 \rho)
$$

and

$$
\frac{m(p-\kappa)}{2}\left(\mu^{\vee}, \mu^{\vee}+2 \rho^{\vee}\right)_{(L \mathfrak{h})^{*}}=\left(-\frac{\kappa}{2}+\frac{p}{2}\right)\left(\mu^{\vee}, \mu^{\vee}+2 \rho^{\vee}\right)
$$


respectively. Therefore conformal dimension of the highest weight vector of the $\left(\lambda, \mu^{\vee}\right)$-term of the vertex algebra 5.2 is equal to

$$
\begin{aligned}
\frac{1}{2}\left(\lambda-\mu^{\vee}, \lambda-\mu^{\vee}\right)+\frac{m q-1}{2}(\lambda, \lambda)+(m q(\lambda, \rho) & \left.-\left\langle\lambda, \rho^{\vee}\right\rangle\right) \\
& +\frac{p-1}{2}\left(\mu^{\vee}, \mu^{\vee}\right)+\left(p\left(\mu^{\vee}, \rho^{\vee}\right)-\left\langle\rho, \mu^{\vee}\right\rangle\right) .
\end{aligned}
$$

The first term is always non-negative, the second and third terms are non-negative (resp. strictly positive) if $q \geq 1$ (resp. $q>1$ ), and the fourth and fifth terms are non-negative (resp. strictly positive) if $p \geq 1$ (resp. $p>1$ ).

We conclude that conformal dimensions in $X_{p, q}(G)$ are bounded from below (in fact, are non-negative) if and only if $p, q \geq 1$. Furthermore, they are strictly positive, with the exception of the vacuum vector (which appears in the $\lambda=\mu=0$ sector) if in addition either $p$ or $q$ is greater than 1 .

Applying the localization functor to $X_{p, q}(G)$, we obtain a twisted $D$-module on the product $\operatorname{Bun}_{G} \times \operatorname{Bun}_{L_{G}}$. The twisting is $\kappa /(m q \kappa-1)$ along the first factor and $1 / m(p-\kappa)$ along the second factor. This should be a kernel of the qGL functor corresponding to the duality transformation $\widetilde{T}^{p} S \widetilde{T}^{q}$ :

$$
\mathcal{E}_{\kappa /(1-m q \kappa)}^{G, \widetilde{T}^{p} S \widetilde{T}^{q}}: D_{\kappa /(1-m q \kappa)}\left(\operatorname{Bun}_{G}\right) \rightarrow D_{1 / m(p-\kappa)}\left(\operatorname{Bun}_{L_{G}}\right) .
$$

Making a change of variables, we can rewrite it as the kernel of

$$
\mathcal{E}_{\kappa}^{G, g}: D_{\kappa}\left(\operatorname{Bun}_{G}\right) \rightarrow D_{g(\kappa)}\left(\operatorname{Bun}_{L_{G}}\right)
$$

where $g=\widetilde{T}^{p} S \widetilde{T}^{q}$ and $g(\kappa)=(m q \kappa+1) / m(m p q \kappa+p-\kappa)$.

\section{Branes, twisted $D$-Modules, and COMPaCtification Functors}

In this section we discuss in more detail the compactification functors and their behavior under the quantum Langlands dualities. We also review the connections between the $D$ modules obtained via compactification functors and the corresponding branes on the Hitchin moduli spaces. Much of the material of this section on the links between branes, $D$-modules, and conformal blocks appeared previously in [55, 59, 35, 42, 36, 37, 9]. A new ingredient is identifying, under some mild conditions, the compactification functor with the localization functor for a junction vertex algebra (up to tensoring with a line bundle on $\operatorname{Bun}_{G}$ ).

6.1. Branes associated to boundary conditions. In their pioneering work [55], Kapustin and Witten introduced and studied the categories of $A$ - and $B$-branes on $\mathcal{M}_{H}(G, X)$ and $\mathcal{M}_{H}\left({ }^{L} G, X\right)$, and the equivalence (homological mirror symmetry) between them that is a consequence of the $S$-duality of the $4 \mathrm{~d}$ gauge theories $\mathcal{T}_{0}^{G}$ and $\mathcal{T}_{\infty}^{L} G$ (in the notation introduced in Section 3). In particular, they studied the following branes, which can be viewed as $A$ - or $B$-branes in each of the three complex structures of the Hitchin moduli spaces, relative to the hyperKäler structure described in [55]:

(i) The zero-branes $\mathbf{B}_{\mathcal{E}}$ - supported at a single (smooth) point $\mathcal{E}$ of $\mathcal{M}_{H}\left({ }^{L} G, X\right)$, each of them has type $(B, B, B)$; 
(ii) Pairs $\left(\mathbf{F}_{b}, \nabla\right)$, where $\mathbf{F}_{b}$ is a smooth fiber table of the Hitchin fibration of $\mathcal{M}_{H}(G, X)$ and a $\nabla$ is a flat $U(1)$-bundle on it - these are mirror dual to the zero-branes $\mathbf{B}_{\varepsilon}$ from (i) and have type $(B, A, A)$.

(iii) The canonical coisotropic brane $\mathcal{B}_{\text {c.c. }}$ - it is supported on the entire $\mathcal{M}_{H}(G, X)$ and has type $(A, B, A)$;

(iv) The space-filling $(B, B, B)$ brane $\widetilde{\mathcal{B}}$ on $\mathcal{M}_{H}\left({ }^{L} G, X\right)$;

(v) The brane of opers $\mathcal{B}_{\mathrm{Op}}$ - it is supported on the subspace of ${ }^{L} G$-opers in $\mathcal{M}_{H}\left({ }^{L} G, X\right)$, has type $(A, B, A)$, and is mirror dual to $\mathcal{B}_{\text {c.c. from (iii); }}$

(vi) The $(B, A, A)$ brane $\mathcal{B}_{\text {cl.op }}$ of "classical opers" (also known as the Hitchin's section) on $\mathcal{M}_{H}(G, X)$ - it is mirror dual to the space-filling $(B, B, B)$ brane $\widetilde{\mathcal{B}}$ from (iv).

To make contact with the formalism of the previous section, we note that to each half-BPS boundary condition $B$ in the physical $4 \mathrm{~d}$ theory we can associate a topological boundary condition in the $\mathcal{T}_{\kappa}^{G}$ theory with $\kappa=0$ or $\infty$. One can also associate to it two families of branes on the Hitchin moduli space $\mathcal{M}_{H}(G)$ : one corresponds to $\kappa=\infty$ and consists of $(B, B, B)$ branes; the other corresponds to $\kappa=0$ and consists of $(B, A, A)$ branes. They arise from two different types of compactifications of the $4 \mathrm{~d}$ theory on the Riemann surface $X$, preserving two different $S O(3)$ subgroups of the group $S O(6)$ of $R$-symmetries of the $4 \mathrm{~d}$ theory (this is explained in detail in [36, 37]).

For a generic $B$, this is the end of the story. But for some special boundary conditions, one of the two types of branes deform to other values of $\kappa$. If a brane does deform, then the deformed brane always has type $(A, B, A)$.

In the above table, the only branes that can be deformed are (iv) and (vi). The former,

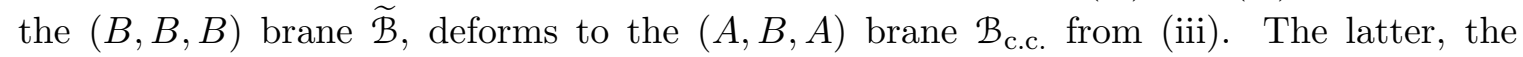
$(B, A, A)$ brane $\mathcal{B}_{\text {cl.Op }}$, deforms to the $(A, B, A)$ brane $\mathcal{B}_{\text {Op }}$ from $(\mathrm{v})$.

Table 3 organizes the branes according to the boundary conditions to which they are associated (we only consider the basic ones: Dirichlet, Neumann, and principal Nahm).

\begin{tabular}{|c||c||c||c||c||c|}
\hline Boundary Condition & $\kappa$ & Brane & Type & Deforms? & If yes, D-mod \\
\hline \hline Dirichlet $\left(D_{0,1}\right)$ & $\infty$ & $\mathbf{B}_{\mathcal{E}}$ & $(B, B, B)$ & no & \\
\hline Dirichlet $\left(D_{0,1}\right)$ & 0 & $\mathcal{F}_{\mathcal{P}}$ & $(B, A, A)$ & yes, $\mathcal{F}_{\mathcal{P}}^{\prime}$ & $\delta_{\mathcal{P}}^{\kappa}$ \\
\hline Neumann $\left(N_{1,0}\right)$ & $\infty$ & $\widetilde{\mathcal{B}}^{x_{i}, \lambda_{i}}$ & $(B, B, B)$ & yes, $\mathcal{B}_{\text {c.c. }}^{x_{i}, \lambda_{i}}$ & $D_{\kappa}^{x_{i}, \lambda_{i}}$ \\
\hline Neumann $\left(N_{1,0}\right)$ & 0 & $\mathcal{B}_{0}^{x_{i}, \lambda_{i}}$ & $(B, A, A)$ & no & \\
\hline Nahm $\left(N_{0,1}\right)$ & $\infty$ & $\mathcal{B}_{\text {cl.Op }}^{x_{i}, \mu_{i}^{\vee}}$ & $(B, B, B)$ & yes, $\mathcal{B}_{\text {Op }}^{x_{i}, \mu_{i}^{\vee}}$ & $\Psi_{\kappa}^{x_{i}, \mu_{i}^{\vee}}$ \\
\hline $\operatorname{Nahm~}\left(N_{0,1}\right)$ & 0 & Nahm pole of $\sigma$ & $(B, A, A)$ & no & \\
\hline
\end{tabular}

TABLE 3 . The basic boundary conditions, branes and $D$-modules

Note that three of the branes in the middle column, namely, (i), (iv) and (vi), appeared in the above list; as did two of the branes in the rightmost column: (iii) and (v). The branes 
$\left(\mathbf{F}_{b}, \nabla\right)$ from (ii) correspond to the dual of the $(B, B, B)$ Dirichlet boundary conditions from (i) and hence do not appear in our table.

Although the $(B, B, B)$ Dirichlet and their duality images are not the $\kappa \rightarrow q / p$ limits of the $D_{p, q}$ boundary conditions which exist for irrational $\kappa$, the vertex algebra technology can still be used to explore their properties. We will comment on this observation only briefly here, in Section 6.7, and leave a more detailed discussion to future work.

Now we give more details on the branes appearing in Table 3.

6.1.1. Dirichlet boundary condition. In this case, the $(B, B, B)$ branes are the zero-branes $\mathbf{B}_{\varepsilon}$ which appeared in [55]. They cannot be deformed away from $\kappa=\infty$ (this can be seen from the fact that the deformation of $\mathcal{M}_{H}\left({ }^{L} G\right)$ away from $\kappa=\infty$ is non-commutative, so points of $\mathcal{M}_{H}\left({ }^{L} G\right)$ no longer make sense). Likewise, the branes $\left(\mathbf{F}_{b}, \nabla\right)$, supported on the fibers of the Hitchin fibration, which are mirror dual to the zero-branes $\mathbf{B}_{\mathcal{E}}$, as shown in [55. They cannot be deformed away from $\kappa=0$.

The $(B, A, A)$ branes associated to the Dirichlet boundary condition are the branes $\mathcal{F}_{\mathcal{P}}$ on $\mathcal{M}_{H}(G)$, where $\mathcal{P}$ is a semi-stable $G$-bundle on $X$. Recall that in the complex structure $I$ the Hitchin moduli space is identified with the moduli space of semi-stable Higgs bundles on $X$. Hence it contains as an open subspace the holomorphic cotangent bundle to the moduli space $\mathcal{M}(G, X)$ of semi-stable $G$-bundles. The brane $\mathcal{F}_{\mathcal{P}}$ is supported on the fiber of this cotangent bundle at $\mathcal{P}$ (this map was called "the second Hitchin fibration" in [55] to distinguish it from the Hitchin map to the Hitchin base). Note that since these fibers are vector spaces, they do not support any non-trivial flat connections. Each brane $\mathcal{F}_{\mathcal{P}}$ has type $(B, A, A)$.

The branes $\mathcal{F}_{\mathcal{P}}$ can be deformed away from $\kappa=0$ to the $(A, B, A)$ branes which we denote by $\mathcal{F}_{\mathcal{P}}^{\prime}$. These are constructed in a similar fashion, but using the complex structure $J$, in which $\mathcal{M}_{H}(G, X)$ appears as the moduli space of semi-stable flat $G$-bundles on $X$. Then an open part of $\mathcal{M}_{H}(G, X)$ also maps to $\mathcal{M}(G, X)$, and the fibers of this map give rise to a second family of branes, which we denote by $\mathcal{F}_{\mathcal{P}}^{\prime}$, where again $\mathcal{P} \in \mathcal{M}(G, X)$ (these are affine spaces, hence they do not support any non-trivial flat connections). Note that the fibers of this map are different from the fibers of the previous map, and hence the branes $\mathcal{F}_{\mathcal{P}}^{\prime}$ are different from $\mathcal{F}_{\mathcal{P}}$.

The $\kappa$-twisted $D$-modules on Bun $_{G}$ associated to the above "fiber branes" are the $\delta$ function $D$-modules $\delta_{\mathcal{P}}^{\kappa}$ discussed in the previous section. More precisely, $\mathcal{F}_{\mathcal{P}}$ corresponds to $\delta_{\mathcal{P}}^{0}$ for $\kappa=0$ and $\mathcal{F}_{\mathcal{P}}^{\prime}$ corresponds to $\delta_{\mathcal{P}}^{\kappa}$ for $\kappa \neq 0$.

Unlike the branes that are mirror dual to the $(B, B, B)$ zero-branes $\mathbf{B}_{\mathcal{E}}$, which have been described in [55] in the case when $\mathcal{E}$ is a smooth point of $\mathcal{M}_{H}\left({ }^{L} G, X\right)$ (these are the branes $\left(\mathbf{F}_{b}, \nabla\right)$ from supported on the fibers of the first Hitchin fibration), the mirror dual branes to the $(B, A, A)$ branes $\mathcal{F}_{\mathcal{P}}$, and to their deformations $\mathcal{F}_{\mathcal{P}}^{\prime}$, are rather complicated, and no explicit description for them is presently known (apart from a small number of cases, including that of abelian $G$ ). However, as we argue in this paper, we can construct vertex algebras which give rise to the twisted $D$-modules corresponding to these complicated branes using the localization functor to $\operatorname{Bun}_{G}$. 
6.1.2. Neumann boundary condition. Then the $(B, B, B)$ branes are the space-filling brane $\widetilde{\mathcal{B}}$ and its generalizations $\widetilde{\mathcal{B}}^{x_{i}, \lambda_{i}}$ obtained by applying the Wilson line operators corresponding to the dominant integral weights $\lambda_{i}$ of $G$ at the points $x_{i} \in X$ to $\widetilde{\mathcal{B}}$. These $(B, B, B)$ branes deform to the $(A, B, A)$ brane $\mathcal{B}_{\text {c.c }}$ and its generalizations $\mathcal{B}_{\text {c.c }}^{x_{i}, \lambda_{i}}$ on $\mathcal{M}_{H}\left({ }^{L} G, X\right)$ away from $\kappa=\infty$. The corresponding twisted $D$-modules on $\operatorname{Bun}_{L_{G}}$ are $\mathcal{D}_{\kappa}$ (whose limit as $\kappa \rightarrow \infty$ can be identified with the structure sheaf on $\operatorname{Loc}_{L_{G}}$, the moduli stack of flat ${ }^{L} G$-bundles on $X$ ) and its generalizations $\mathcal{D}_{\kappa}^{x_{i}, \lambda_{i}}$.

A Neumann-type brane that does not deform is the $(B, A, A)$ brane $\mathcal{B}_{0}$ which is the zero section of the cotangent bundle $T^{*} \mathcal{N}(G, X) \subset \mathcal{M}_{H}(G, X)$.

6.1.3. Nahm boundary condition. In this case the $(B, A, A)$ branes are the brane $\mathcal{B}_{\text {cl.Op }}$ of "classical opers" on $\mathcal{M}_{H}(G, X)$ and its generalizations $\mathcal{B}_{\text {cl.Op }}^{x_{i}, \mu_{i}^{\vee}}$ obtained by applying the 't Hooft line operators corresponding to the dominant integral coweights $\mu_{i}^{\vee}$ of $G$ at the points $x_{i} \in X$ to $\mathcal{B}_{\text {cl.Op. These }}(B, A, A)$ brane (for the group $G$ ) are mirror dual to the $(B, B, B)$ branes $\widetilde{\mathcal{B}}^{x_{i}, \mu_{i}^{\vee}}$ (for the group ${ }^{L} G$ ), which is consistent with the fact that the category $\mathcal{C}_{0}^{G}\left(N_{0,1}^{G}\right)$ is $S$-dual to $\mathcal{C}_{\infty}^{L} G\left(N_{1,0}^{G}\right)$.

The $(B, A, A)$ brane $\mathcal{B}_{\text {cl.op }}$ deforms away from $\kappa=0$ to the $(A, B, A)$ brane of opers $\mathcal{B}_{\mathrm{Op}}$ on $\mathcal{M}_{H}(G, X)$, and the branes $\mathcal{B}_{\text {cl.Op }}^{x_{i}, \mu_{i}^{\vee}}$ deform to the $(A, B, A)$ branes $\mathcal{B}_{\mathrm{Op}}^{x_{i}, \mu_{i}^{\vee}}$. Note that for irrational $\kappa$ there are no bulk 't Hooft operators, so $\mathcal{B}_{\mathrm{Op}}^{x_{i}, \mu_{i}^{\vee}}$ cannot be obtained by applying 't Hooft operators to $\mathcal{B}_{\mathrm{Op}}$.

The $\kappa$-twisted $D$-modules on $\operatorname{Bun}_{G}$ associated to the branes $\mathcal{B}_{\mathrm{Op}}^{x_{i}, \mu_{i}^{\vee}}$ are the Whittaker sheaves $\Psi_{\kappa}^{x_{i}, \mu_{i}^{\vee}}$ (see Section 3.2, Example (iii)).

This is consistent with the fact that the category $\mathcal{C}_{\kappa}^{G}\left(N_{0,1}^{G}\right)=$ Whit $_{-\kappa}(G)$ is $S$-dual to $\mathcal{C}_{-1 / m \kappa}^{L_{G}}\left(N_{1,0}^{G}\right)=\mathrm{KL}_{-1 / m \kappa}$. The corresponding functor

$$
\varepsilon_{\kappa}^{G, S_{m}}: D_{\kappa}\left(\operatorname{Bun}_{G}\right) \rightarrow D_{-1 / m \kappa}\left(\operatorname{Bun}_{L_{G}}\right)
$$

should send the Whittaker sheaves $\Psi_{\kappa}^{x_{i}, \mu_{i}^{\vee}}$ to the $-1 / m \kappa$-twisted $D$-modules $\mathcal{D}_{-1 / m \kappa}^{x_{i}, \mu_{i}^{\vee}}$ on $\operatorname{Bun}_{L_{G}}$ corresponding to the $(A, B, A)$ brane $\mathcal{B}_{\text {c.c. }}^{x_{i}, \mu_{i}^{\vee}}$.

Finally, the Nahm-type brane that does not deform (which is mirror dual of the above "zero section" Neumann-type $(B, A, A)$ brane) should involve a Nahm pole of additional fields $\sigma$ and $\bar{\sigma}$ that appear in the dimensional reduction of the $4 \mathrm{~d}$ gauge theory but are not usually included among the degrees of freedom of the Hitchin moduli space, see [55, 40, 41] (this boundary condition appears to be related to the Arthur's $S L_{2}$ in the classical Langlands correspondence, see Sect. 6.2 of [26]). It cannot be deformed away from $\kappa=\infty$, just as its mirror dual "zero section" Neumann-type $(B, A, A)$ brane cannot be deformed away from $\kappa=0$. This actually provides an important insight into the expected behavior of the qGL duality functors $\mathcal{E}_{\kappa}^{G, g}$, see Remark 6.1 below.

6.2. Branes vs. D-modules. In [55], Kapustin and Witten described a link between the geometric Langlands correspondence and mirror symmetry of categories of branes on 
the Hitchin moduli spaces $\mathcal{M}_{H}(G, X)$ and $\mathcal{M}_{H}\left({ }^{L} G, X\right)$ in terms of the following triangle of categories:

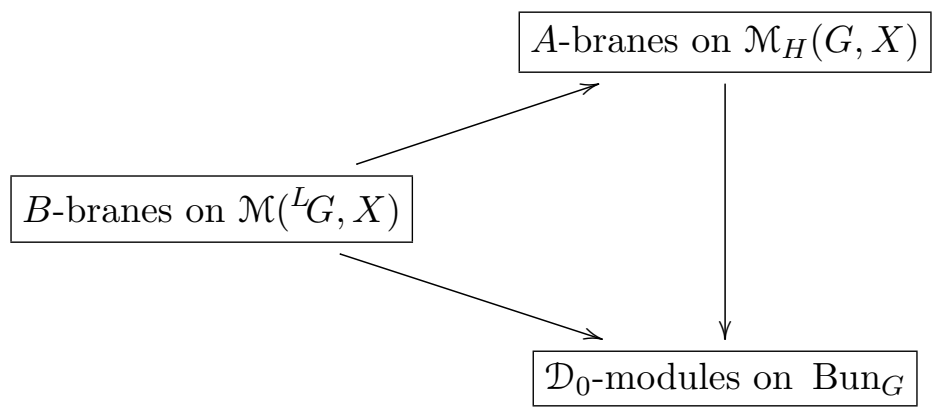

Here $B$-branes on $\mathcal{M}\left({ }^{L} G, X\right)$ are considered in the complex structure $J$, in which $\mathcal{M}\left({ }^{L} G, X\right)$ is viewed as the moduli space $y\left({ }^{L} G\right)$ of semi-stable ${ }^{L} G$-local systems on $X$. This category is usually interpreted mathematically as the (derived) category of coherent sheaves on $y\left({ }^{L} G\right)$. Hence it is closely related to the (derived) category of coherent sheaves (or, equivalently, O-modules) on $\operatorname{Loc}_{L} G$, the moduli stack of flat ${ }^{L} G$-bundles on $X$. For example, the two categories share some familiar objects: zero-branes $\mathbf{B}_{\mathcal{E}}$ on $\mathcal{M}\left({ }^{L} G, X\right)$ (example (i) from Section 6.1) correspond to the skyscraper sheaves on $\operatorname{Loc}_{L_{G}}$, and the space filling $B$-brane on $\mathcal{M}\left({ }^{L} G, X\right)$ (example (iv) from Section 6.1) corresponds to the structure sheaf on $\operatorname{Loc}_{L_{G}}$. However, there are two important differences between the two categories: first, $\operatorname{Loc}_{L_{G}}$ is the moduli stack of flat ${ }^{L} G$-bundles on $X$, whereas $\mathcal{M}_{H}\left({ }^{L} G, X\right)$ in complex structure $J$ is the moduli space $y\left({ }^{L} G\right)$ of semi-stable ones. Second, from the physics perspective it is more natural to consider coherent sheaves on $y\left({ }^{L} G\right)$ with respect to its complex analytic rather than algebraic structure, whereas in the traditional formulation of the geometric Langlands correspondence one considers algebraic $\mathcal{O}$-modules on $\operatorname{Loc}_{L_{G}}$ (see however, [19]).

The upper arrow in the diagram (6.1) represents the (homological) mirror symmetry, while the lower arrow represents the geometric Langlands correspondence (up to the above two subtleties).

The vertical arrow linking the two should be viewed, according to [55], as an equivalence of two (derived) categories that is independent from both mirror symmetry and geometric Langlands. Rather, it is meant to be a special case of a general link between the (derived) categories of $\mathcal{D}$-modules on a variety $M$ (twisted by a square root of the canonical line bundle on $M$ ) and $A$-branes on its cotangent bundle $T^{*} M$ (indeed, $\mathcal{M}_{H}(G)$ is closely connected to $\left.T^{*} \operatorname{Bun}_{G}\right)$. We refer the reader to [55] for more details.

The picture summarized above corresponds to the case of $\kappa=0$ on the $G$-side and $\check{\kappa}=\infty$ on the ${ }^{L} G$-side of the geometric Langlands correspondence. In [55], a deformation of this picture to non-zero values of $\kappa$ was considered as well. In this deformation, one gets a link between the $\kappa$-deformed version of the above categorical mirror symmetry and a quantum deformation of the geometric Langlands duality.

This is a beautiful idea that has led to important developments in mathematics aiming at rigorously establishing equivalences of this kind between categories of $A$-branes and $\mathcal{D}$ modules (or constructible sheaves). However, in the context of the above diagram there still 
remain several obstacles to making a precise mathematical statement about such an equivalence. One of them is the difference between the variety $\mathcal{M}_{H}(G)$ and the stack $T^{*} \operatorname{Bun}_{G}$. The other is that in order to have an equivalence between the categories connected by the lower arrow one needs to modify one of these categories in a non-trivial way (see [6, 20, for details).

Thus, while the diagram (6.1) offers a useful perspective on the links between $S$-duality (or mirror symmetry) and geometric Langlands, the technical difficulties involved in making it mathematically precise encourages one to look for alternative proposals.

In this paper we make such an proposal. We show how to use a junction of a given boundary condition $B$ with the Dirichlet boundary condition (in the bulk theory $\mathcal{T}_{\kappa}^{G}$ ) to directly construct the compactification functor from the category of line defects associated to $B$ to a category of $\kappa$-twisted $D$-modules on $\operatorname{Bun}_{G}$, bypassing the categories of branes. Thus, at the outset, we consider the boundary conditions and the corresponding categories of line defects separately from one other. A priori, we only expect that the groupoid of quantum dualities of the $4 \mathrm{~d}$ gauge theory acts by equivalences on the categories of line defects associated to $B$. The corresponding functors on the categories of twisted $D$-modules on $\operatorname{Bun}_{G}$ arise a posteriori, as functors that should intertwine the compactification functors. A priori there is no reason to expect these functors to be equivalences (and in fact we only expect that to be true for irrational $\kappa$ ). This understanding removes seeming inconsistencies between the statements that are made in $4 \mathrm{~d}$ gauge theory and in the mathematical theory of quantum geometric Langlands duality (see Remark 6.1 at the end of the next subsection for more details).

6.3. From boundary conditions to twisted $D$-modules. The starting point of our proposal is that for each boundary condition $B$, we have the category of line defects $\mathcal{C}_{\kappa}^{G}(B)$ associated to $B$ in the bulk theory $\mathcal{T}_{\kappa}^{G}$. At the outset, we look at these categories separately (rather than considering all boundary conditions and the corresponding line defects together). For each $B$, we then construct a compactification functor from $\mathcal{C}_{\kappa}^{G}(B)$ to $D_{\kappa}\left(\operatorname{Bun}_{G}\right)$, using a junction $D_{0,1}^{G} \rightarrow B$ from the Dirichlet boundary condition to $B$.

The Dirichlet boundary condition plays a fundamental role in $4 \mathrm{~d}$ gauge theory. In fact, one could argue that it is only after we specify this boundary condition that we fix the gauge group $G_{c}$ of our theory (and hence its complexification $G$ ). For the class of twisted gauge theories we are considering, this means that identifying our $4 \mathrm{~d}$ gauge theory with a specific theory $\mathcal{T}_{\kappa}^{G}$.

The traditional approach is to first define $\mathcal{T}_{\kappa}^{G}$ in the weak coupling limit (corresponding to $\kappa$ close to 0 ), and then argue that it can be analytically continued to other values of $\kappa$. But we find that among the resulting theories, there are many equivalences given by the duality groupoid (see Section 3.3). Therefore, a more fruitful point of view might be that each orbit of the duality groupoid gives rise to a single quantum field theory that has many different Lagrangian descriptions (each of them corresponding to a particular weak coupling limit, for a suitably chosen parameter $\kappa$ ). Thus, for example, we see that the gauge group of the theory is not really a well-defined object in a theory; rather, it is a pair of Langlands dual groups that is well-defined (if we include discrete $\theta$-angles, then we get an even larger set of groups, though all of them belong to the isogeny classes of $G$ and ${ }^{L} G$ ). 
To summarize, given a quantum $4 \mathrm{~d}$ gauge theory, we have a choice which boundary condition (among many potential candidates) to call the Dirichlet boundary condition $D_{0,1}^{G}$ of our theory. This choice implies a particular Lagrangian (weakly coupled) description, and hence an identification of the theory as $\mathcal{T}_{\kappa}^{G}$ for particular $G$ and $\kappa$.

Now, suppose we have declared our choice of $D_{0,1}^{G}$ (which includes the choice of the corresponding group $G$ ). The Dirichlet boundary condition $D_{0,1}^{G}$ has a special property: it has a global symmetry group, which is nothing but the gauge group $G_{c}$ of the theory $\mathcal{T}_{\kappa}^{G}$. This allows us to couple this boundary condition to a background $G$-bundle on the boundary. Suppose that the boundary is the product of a Riemann surface $X$ and a real line. Then we can couple this boundary condition to a $G$-bundle $\mathcal{P}$ on $X$ extended to this product. Given another boundary condition $B$ together with a line defect (i.e. an object $\mathcal{A}$ of $\mathcal{C}_{\kappa}^{G}(B)$ ), we can then define a family of vector spaces $\mathbf{H}_{\mathcal{P}}^{B}(\mathcal{A})$ labeled by $\mathcal{P} \in \operatorname{Bun}_{G}$. Furthermore, one can argue on general grounds of TFT that these vector spaces must be the fibers of a $\kappa$-twisted $D$-module on $\operatorname{Bun}_{G}$, or more generally an object of the derived category $D_{\kappa}\left(\operatorname{Bun}_{G}\right)$. For simplicity, we refer to all of them simply as $\kappa$-twisted $D$-modules on $\operatorname{Bun}_{G}$. This way we assign to $B$ and $\mathcal{A}$ a $\kappa$-twisted $D$-module on $\operatorname{Bun}_{G}$, and this construction gives rise to the compactification functor from $\mathcal{C}_{\kappa}^{G}(B)$ to $D_{\kappa}\left(\operatorname{Bun}_{G}\right)$.

Let us explain this more precisely. What follows may be viewed a motivation from the $4 \mathrm{~d}$ gauge theory perspective. We will give a rigorous mathematical definition of the compactification functor (under a few assumptions) in Section 6.5 below.

(1) Let $B$ be a boundary condition in the theory $\mathcal{T}_{\kappa}^{G}$ and $X$ a Riemann surface. Consider the $4 \mathrm{~d}$ theory $\mathcal{T}_{\kappa}^{G}$ on the product of $X$ and an infinite strip, with the Dirichlet boundary condition $D_{0,1}^{G}$ on one side and $B$ on the other side. Let $\mathcal{P}$ be a $G$-bundle on $X$, which we extend to the product of $X$ and the real line. Let's couple the Dirichlet boundary condition to this $G$-bundle. In the limit in which the width of the strip goes to zero, this yields a $3 \mathrm{~d}$ TFT, whose space of states on $X$ we denote by $\mathbf{H}_{\mathcal{P}}^{B}$. On general grounds, as explained in Section B.2 of [36], we expect the spaces $\mathbf{H}_{\mathcal{P}}^{B}$ for different $\mathcal{P} \in \operatorname{Bun}_{G}$ to be the fibers of a $\kappa$-twisted $D$-module on $\operatorname{Bun}_{G}$ (the action of vector fields comes from a version of the "Berry connection" familiar to physicists). This is the image $F_{\kappa}^{G, B}(I)$ of the compactification map.

(2) Consider the same configuration as above, but with a line defect corresponding to an object $\mathcal{A}$ of the category $\mathcal{C}_{\kappa}^{G}(B)$ on the boundary of the strip on which we have the boundary condition $B$, situated at $x \in X$ (see Figure 1). Denote the corresponding space of states, in the limit when the width of the strip goes to zero, by $\mathbf{H}_{\mathcal{P}}^{B}(\mathcal{A})$. Then, for the same reason as in $(1)$, we expect that $\mathbf{H}_{\mathcal{P}}^{B}(\mathcal{A})$ are the fibers of a $\kappa$-twisted $D$-module on $\operatorname{Bun}_{G}$. We denote this $D$-module by $F_{\kappa}^{G, B}(\mathcal{A})$.

(3) Given another object $\mathcal{A}^{\prime}$ of $\mathcal{C}_{\kappa}^{G}(B)$, and a morphism $\mathcal{A} \rightarrow \mathcal{A}^{\prime}$, general properties of TFT give rise to a morphism $F_{\kappa}^{G, B}(\mathcal{A}) \rightarrow F_{\kappa}^{G, B}\left(\mathcal{A}^{\prime}\right)$.

Thus we obtain a functor

$$
F_{\kappa}^{G, B}: \mathcal{C}_{\kappa}^{G}(B) \rightarrow D_{\kappa}\left(\operatorname{Bun}_{G}\right) .
$$


This is the compactification functor. It has a multi-point generalization compatible with fusion and braiding in $\mathcal{C}_{\kappa}^{G}(B)$.

(4) If $g$ is an element of the duality groupoid $\mathcal{G}_{\kappa}^{G}$, then for irrational $\kappa$ we should have an equivalence of categories

$$
\mathfrak{C}_{\kappa}^{G}(B) \simeq \mathrm{C}_{g(\kappa)}^{g(G)}(g(B))
$$

Therefore, it is natural to assume the existence of a quantum GL duality functor

$$
\mathcal{E}_{\kappa}^{G, g}: D_{\kappa}\left(\operatorname{Bun}_{G}\right) \rightarrow D_{g(\kappa)}\left(\operatorname{Bun}_{g(G)}\right)
$$

intertwining the equivalences $(6.2)$ via the corresponding compactification functors $F_{\kappa}^{G, B}$ and $F_{g(\kappa)}^{g(G), g(B)}$ in the sense that they fit in the commutative diagram 4.3. Thus, we arrive at the statement of Conjecture 4.1 .

Remark 6.1. Note that from the point of view of $4 \mathrm{~d}$ gauge theory, we can only claim that the essential images of the compactification functors $F_{\kappa}^{G, B}$ and $F_{g(\kappa)}^{g(G), g(B)}$ for a fixed boundary condition $B$ are equivalent. A priori, there is no claim that the categories $D_{\kappa}\left(\operatorname{Bun}_{G}\right)$ and $D_{g(\kappa)}\left(\operatorname{Bun}_{g(G)}\right)$ are equivalent. On general grounds, we do expect an equivalence between the categories of $1 \mathrm{~d}$ boundary conditions in the compactified $2 \mathrm{~d}$ theories $\mathcal{T}_{\kappa}^{G}[X]$ and $\mathcal{T}_{g(\kappa)}^{g(G)}[X]$, but it is not clear that the category $\mathcal{T}_{\kappa}^{G}[X]$ coincides with $D_{\kappa}\left(\operatorname{Bun}_{G}\right)$. In general, some of the $D$-modules may have to be excluded because of various subtleties with the definition of the theory $\mathcal{T}_{\kappa}^{G}[X]$. On the other hand, some $1 \mathrm{~d}$ boundary conditions may not correspond to any $D$-modules on $\operatorname{Bun}_{G}$.

For irrational $\kappa$, we expect that $D_{\kappa}\left(\operatorname{Bun}_{G}\right)$ is equivalent to the category $\mathcal{T}_{\kappa}^{G}[X]$. Therefore, for irrational $\kappa$ we expect that each functor $\mathcal{E}_{\kappa}^{G, g}$ is an equivalence of categories.

For rational values of $\kappa$, however, we expect that $\mathcal{T}_{\kappa}^{G}[X]$ is different from $D_{\kappa}\left(\operatorname{Bun}_{G}\right)$. Perhaps, a part of it can be described as a certain "tempered" subcategory of $D_{\kappa}\left(\operatorname{Bun}_{G}\right)$. This is motivated by the observation that in the $4 \mathrm{~d}$ gauge theory there are additional fields, denoted by $\sigma$ and $\bar{\sigma}$ in [55, that appear to take care of the parameters for non-tempered $D$ modules. For example, the "constant" $D$-module in $D_{0}\left(\operatorname{Bun}_{G}\right)$ corresponds to the principal Nahm pole for the fields $\sigma$ and $\bar{\sigma}$ (see Section 6.1.3 and [26]). But these fields represent additional degrees of freedom in the dual theory, which suggests that if we only consider boundary conditions in the dual theory in which these fields are set to 0 , then we must also exclude the "constant" $D$-module on Bun $_{G}$, which is a non-tempered object of the category $D_{0}\left(\operatorname{Bun}_{G}\right)[\underline{6}, 20$.

In general, it is usually difficult to explicitly compute the spaces $\mathbf{H}_{\mathcal{P}}^{B}(\mathcal{A})$, appearing in part (3) above, purely in terms of the $4 \mathrm{~d}$ gauge theory (or the $3 \mathrm{~d}$ TFT obtained by its dimensional reduction). And that's where vertex algebras come to the rescue. In fact, it turns out that the compactification functor for a boundary condition $B$ can be described rigorously mathematically (under some assumptions) as the localization functor for modules over the vertex algebra associated to any non-degenerate junction $D_{0,1}^{G} \rightarrow B$ (possibly, up to tensoring with a power of the line bundle $\mathcal{L}_{G}$ on $\operatorname{Bun}_{G}$ ). In order to explain this, we first recall the concepts of coinvariants, conformal blocks, and the localization functor. 
6.4. Generalities on conformal blocks and localization functors. Here we recall the notions of localization functors, conformal blocks, and coinvariants (see [28], Ch. 18 for details; for a brief survey aimed at physicists, see Sect. 7 of [25]).

Let $V$ be a vertex algebra and $\mathcal{A}_{g, n}$ the category whose objects are $\left(X,\left(x_{i}\right),\left(M_{i}\right)\right)$, where $\left(X,\left(x_{i}\right)_{i=1, \ldots, n}\right)$ is an $n$-punctured compact Riemann surface (more generally, a stable $n$ pointed curve) and $\left(M_{i}\right)_{i=1, \ldots, n}$ is an ordered set of $V$-modules (we think of each $M_{i}$ as attached to the point $x_{i}$ ), and morphisms are holomorphic maps. Recall [64] that a modular functor $\mathcal{H}$ is a functor from the category $\mathcal{A}_{g, n}$ to the category of vector spaces:

$$
\left(X,\left(x_{i}\right),\left(M_{i}\right)\right) \quad \mapsto \quad H_{V}\left(X,\left(x_{i}\right),\left(M_{i}\right)\right),
$$

satisfying the well-known "sewing axiom" (see [64 for details). The vector space on the right hand side of (6.4) is the space of coinvariants, which is dual to the space of conformal blocks (see [28, 25] for the precise definition of these spaces for any vertex algebra and a given collection $\left(X,\left(x_{i}\right),\left(M_{i}\right)\right)$ as above).

Next, suppose that $V$ is a vertex algebra containing as a subalgebra the vertex algebra $V_{\kappa}(\widehat{\mathfrak{g}})$ of an affine Kac-Moody algebra $\widehat{\mathfrak{g}}$. In this case, we will say that $V$ has $\widehat{\mathfrak{g}}$-symmetry of level $\kappa$. Then the space of coinvariants $H_{V}\left(X,\left(x_{i}\right),\left(M_{i}\right)\right.$ ) (as well as its dual) may be twisted by an arbitrary $G$-bundle $\mathcal{P}$ on $X$ (see [28, 25]).

For instance, let $V=V_{\kappa}(\mathfrak{g})$, the affine Kac-Moody algebra $\widehat{\mathfrak{g}}$ of (shifted) level $\kappa$. Then the ordinary (untwisted) space of coinvariants is the quotient

$$
H\left(X,\left(x_{i}\right),\left(M_{i}\right)\right)=\otimes_{i} M_{i} /\left(\mathfrak{g}_{\text {out }} \cdot \otimes_{i} M_{i}\right),
$$

where

$$
\mathfrak{g}_{\text {out }}=\mathfrak{g} \otimes \mathbb{C}\left[X \backslash\left\{x_{1}, \ldots, x_{n}\right\}\right]
$$

(it is viewed as a Lie subalgebra in the direct sum of $n$ copies of $\widehat{\mathfrak{g}}$ with their centers identified). Its dual space is the ordinary space of conformal blocks:

$$
\mathcal{C}\left(X,\left(x_{i}\right),\left(M_{i}\right)\right)=\operatorname{Hom}_{\mathfrak{g}_{\text {out }}}\left(\otimes_{i} M_{i}, \mathbb{C}\right),
$$

Now let $\mathcal{P}$ be a $G$-bundle on $X$. Then we define the space of $\mathcal{P}$-twisted coinvariants as

$$
H^{\mathcal{P}}\left(X,\left(x_{i}\right),\left(M_{i}\right)\right)=\otimes_{i} M_{i} /\left(\mathfrak{g}_{\text {out }}^{\mathcal{P}} \cdot \otimes_{i} M_{i}\right)
$$

where

$$
\mathfrak{g}_{\text {out }}^{\mathcal{P}}=H^{0}\left(X \backslash\left\{x_{1}, \ldots, x_{n}\right\}, \mathcal{P} \underset{G}{\times} \mathfrak{g}\right) .
$$

Its dual space is the space of $\mathcal{P}$-twisted conformal blocks:

$$
\mathcal{C}^{\mathcal{P}}\left(X,\left(x_{i}\right),\left(M_{i}\right)\right)=\operatorname{Hom}_{\mathfrak{g}_{\text {out }}^{\mathcal{P}}}\left(\otimes_{i} M_{i}, \mathbb{C}\right),
$$

For a general vertex algebra $V$, to obtain the space of coinvariants (or conformal blocks), we take the quotient (or take invariant functionals) with respect to a larger Lie algebra, encompassing all Ward identifies of all fields of the vertex algebra $V$. If $V$ has $\widehat{\mathfrak{g}}$-symmetry, then this Lie subalgebra can be twisted by a $G$-bundle $\mathcal{P}$ on $X$. This way, we obtain the $\mathcal{P}$-twisted spaces of coinvariants and conformal blocks, $\mathcal{H}_{V}^{\mathcal{P}}\left(X,\left(x_{i}\right),\left(M_{i}\right)\right)$ and $\mathcal{C}_{V}^{\mathcal{P}}\left(X,\left(x_{i}\right),\left(M_{i}\right)\right)$, see [28. Thus, we obtain a modular functor $\mathcal{H}_{V}^{\mathcal{P}}$, and its conformal blocks version $\mathcal{C}_{V}^{\mathcal{P}}$, for each $G$-bundle $\mathcal{P}$. It is natural to call it a $\mathcal{P}$-twist of $\mathcal{H}$ (or $\mathcal{C}$ ). 
As shown in [28], if the modules $M_{i}$ are such that the action of the Lie subalgebra $\mathfrak{g}[[z]]$ of $\widehat{\mathfrak{g}}$ extends to the group $G[[z]]$, then the spaces of $\mathcal{P}$-twisted coinvariants are fibers (or stalks) of a naturally defined $\kappa$-twisted $D$-module on $\operatorname{Bun}_{G}$. This $\kappa$-twisted $D$-module is obtained by applying the so-called localization functor $\Delta_{\kappa}$ to $\otimes_{i} M_{i}$.

More generally, suppose that the action of the Lie subalgebra $z^{m_{i}} \mathfrak{g}[[z]]$ of $\widehat{\mathfrak{g}}$ extends to the corresponding subgroup of $G[[z]]$, for some $m_{i} \geq 0$. Then we obtain a twisted $D$-module on $\operatorname{Bun}_{G,\left(x_{i}\right),\left(m_{i}\right)}$, the moduli stack classifying the data $\left(\mathcal{P},\left(\eta_{i}\right)\right)$, where $\mathcal{P}$ is a $G$-bundle on $X$ and $\eta_{i}$ is a trivialization of $\mathcal{P}$ on the $\left(m_{i}-1\right)$ st formal neighborhood of the point $x_{i}$. If $M_{i}$ is a highest weight $\widehat{\mathfrak{g}}$-module (or a module from the category $\mathcal{O}$ ), then we can replace the trivialization of the fiber $\mathcal{P}_{x_{i}}$ of $\mathcal{P}$ at $x_{i}$ (which corresponds to the case $m_{i}=1$ ) by a parabolic structure, that is a reduction of $\mathcal{P}_{x_{i}}$ to a Borel subgroup of $G$. In this paper, we restrict ourselves to the case $m_{i}=0$, so our localization functors takes values in the category of $\kappa$-twisted $D$-modules on $\operatorname{Bun}_{G}=\operatorname{Bun}_{G,\left(x_{i}\right),(0)}$.

Now recall (see, e.g., [56, 50]) that for a twisted $D$-module $\mathcal{R}$ on a smooth manifold $Z$, the stalk of $\mathcal{D}$ at a point $p \in Z$ is defined as

$$
i_{p}^{*}(\mathcal{R})=\mathcal{R} \underset{\mathcal{O}_{Z}}{\otimes} \mathbb{C}_{p},
$$

where $\mathbb{C}_{p}$ is the skyscraper sheaf supported at $p$; that is, the $\mathcal{O}_{Z}$-module $\mathbb{C}_{p}=\mathcal{O}_{Z} / \mathfrak{m}_{p}$, where $\mathfrak{m}_{p}$ is the sheaf of functions vanishing at $p$.

We can rewrite this as follows:

$$
i_{p}^{*}(\mathcal{R})=\mathcal{R} \underset{\mathcal{D}_{Z}}{\otimes} \delta_{p}
$$

The functor $i_{p}^{*}$ is right exact. One can show that the left derived functor of $i_{p}^{*}$ is equivalent to the right derived functor of the functor $i_{p}^{!}$coupled with cohomological shift by $-\operatorname{dim} Z$, where

We will ignore this cohomological shift.

$$
i_{p}^{!}(\mathcal{R})=\operatorname{Hom}_{\mathcal{O}_{Z}}\left(\mathbb{C}_{p}, \mathcal{R}\right)=\operatorname{Hom}_{\mathcal{D}_{Z}}\left(\delta_{p}, \mathcal{R}\right) .
$$

If the category of $D$-modules on $Z$ twisted by the square root of the canonical line bundle on $Z$ is equivalent to the category of $A$-branes on $T^{*} Z$, then the latter Hom can be described as a Hom in the category of $A$-branes on $T^{*} Z$ (and similarly, for the categories of twisted $D$-modules). And that's because under such an equivalence, the $A$-brane corresponding to the $D$-module $\delta_{p}$ to be $\mathcal{F}_{p}$, the fiber of $T^{*} Z$ over $p \in Z$. So, if $\mathcal{B}$ is the $A$-brane corresponding to the $D$-module $\mathcal{R}$, then we find that isomorphic to

$$
\operatorname{Hom}\left(\delta_{p}, \mathcal{R}\right) \simeq \operatorname{Hom}\left(\mathcal{F}_{p}, \mathcal{B}\right)
$$

up to a shift of cohomological degrees. (We will ignore this cohomological shift as well.)

Therefore, for a semi-stable $G$-bundle $\mathcal{P}$ we can compare the space of $\mathcal{P}$-twisted coinvariants with a Hom of $A$ branes (with respect to the symplectic structure $\omega_{K}$ ):

$$
\mathcal{H}^{\mathcal{P}}\left(X,\left(x_{i}\right),\left(M_{i}\right)\right)=\operatorname{Hom}\left(\delta_{\mathcal{P}}, \Delta\left(\otimes_{i} M_{i}\right)\right) \simeq \operatorname{Hom}\left(\mathcal{F}_{\mathcal{P}}, \mathcal{B}\right),
$$

if $\mathcal{B}$ is the $A$-brane on $\mathcal{M}_{H}(G)$ corresponding to the $D$-module $\Delta\left(\otimes_{i} M_{i}\right)$ obtained by the localization functor from $\otimes_{i} M_{i}$.

This shows that the branes $\mathcal{F}_{\mathcal{P}}$ can be used to link the spaces of $\mathcal{P}$-twisted coinvariants and Hom's in the categories of $D$-modules and $A$-branes. 
If our $\mathcal{D}$-module is holonomic (i.e. all of its fibers are finite-dimensional), we can express the dual space to the space of coinvariants, the space of $\mathcal{P}$-twisted conformal blocks, as a Hom between $D$-modules in the opposite direction (and without any cohomological shift):

$$
\mathcal{C}_{V}^{\mathcal{P}}\left(X,\left(x_{i}\right),\left(M_{i}\right)\right) \simeq \operatorname{Hom}\left(\Delta\left(\otimes_{i} M_{i}\right), \delta_{\mathcal{P}}\right) .
$$

Alternatively, we can write it in terms of the corresponding $A$-brane $\mathcal{B}$, which in this case should be Lagrangian:

$$
\mathcal{C}_{V}^{\mathcal{P}}\left(X,\left(x_{i}\right),\left(M_{i}\right)\right) \simeq \operatorname{Hom}\left(\mathcal{B}, \mathcal{F}_{\mathcal{P}}\right) .
$$

Similarly, we can use the branes $\mathcal{F}_{\mathcal{P}}^{\prime}$ away from the critical level.

6.5. Construction of the compactification functor as a localization functor. Recall that we denote by $D_{0,1}^{G}$ the Dirichlet boundary condition in the $4 \mathrm{~d}$ gauge theory $\mathcal{T}_{\kappa}^{G}$. Let $B$ be another boundary condition in the same theory. Suppose that we have a specific junction $D_{0,1}^{G} \rightarrow B$ which is non-degenerate in the sense described in Section 2.2. Then, according to the general formalism, we have the junction vertex algebra $V_{\kappa}^{G}\left(D_{0,1}^{G \rightarrow B}\right)$. From the point of view of the strip geometry discussed in Section 6.3, the vertex algebra $V_{\kappa}^{G}\left(D_{0,1}^{G} \rightarrow B\right)$ is a "boundary chiral algebra" of the $3 \mathrm{~d}$ TFT. On general grounds, we should expect a close relationship between the spaces of conformal blocks of this boundary chiral algebra and the spaces of states $\mathbf{H}_{\mathcal{P}}^{B}(\mathcal{A})$ of the TFT discussed above. In this subsection, we use this idea to give a mathematically rigorous definition of the compactification functor $F_{\kappa}^{G, B}$ as a localization functor, under specific assumptions.

To motivate it, recall that in Section 6.3 we have argued that the vector spaces $\mathbf{H}_{\mathcal{P}}^{B}(\mathcal{A})$ should be fibers of a $\kappa$-twisted $D$-module on $\operatorname{Bun}_{G}$, which we then took as $F_{\kappa}^{G, B}(\mathcal{A})$. On the other hand, as explained in Section 3.7, we expect that $V_{\kappa}^{G}\left(D_{0,1}^{G} \rightarrow B\right)$ contains the affine Kac-Moody algebra $\widehat{\mathfrak{g}}$ of level $\kappa^{\prime}$ such that $\kappa-\kappa^{\prime}=n(G) \cdot m$ for some $m \in \mathbb{Z}$. Recall also that should be a functor

$$
F_{\kappa, D_{0,1}^{G} \rightarrow B}^{G}: D_{\kappa}\left(\operatorname{Gr}_{G}\right) \otimes \mathcal{C}_{\kappa}^{G}(B) \rightarrow V_{\kappa}^{G}\left(D_{0,1}^{G} \rightarrow B\right)-\bmod .
$$

We now make a list of concrete assumptions that we expect this functor to satisfy:

(i) The functor $F_{\kappa, D_{0,1}^{G} \rightarrow B}^{G}$ is fully faithful (in other words, the junction $D_{0,1}^{G} \rightarrow B$ is non-degenerate).

If this is the case, then slightly abusing notation, we will identify the image of $I \otimes \mathcal{C}_{\kappa}^{G}(B)$ under the functor $(6.6)$ with $\mathcal{C}_{\kappa}^{G}(B)$. Here $I$ is the identity object of $D_{\kappa}\left(\mathrm{Gr}_{G}\right)$; namely, $I=\delta_{\overline{1}}$, where 1 is the coset of the identity element of $G((z))$ in $\operatorname{Gr}_{G}$. Given an object $\mathcal{A}$ of the category $\mathcal{C}_{\kappa}^{G}(B)$, denote the image of $I \otimes \mathcal{A}$ under the functor $F_{\kappa, D_{0,1}^{G} \rightarrow B}^{G}$ also by $\mathcal{A}$. The latter $\mathcal{A}$ is thus a $V_{\kappa}^{G}\left(D_{0,1}^{G} \rightarrow B\right)$-module, and hence a $\widehat{\mathfrak{g}}_{\kappa^{\prime}}$-module.

(ii) The action of the Lie subalgebra $\mathfrak{g}[[z]] \subset \widehat{\mathfrak{g}}_{\kappa^{\prime}}$ on $\mathcal{A}$ can be extended to the corresponding group $G[[z]]$. In other words, $\mathcal{A}$ is a $\left(\widehat{\mathfrak{g}}_{\kappa^{\prime}}, G[[z]]\right)$ Harish-Chandra module. 
(iii) The functor $F_{\kappa, D_{0,1}^{G} \rightarrow B}^{G}$ can be described as follows:

$$
\mathcal{F} \in D_{\kappa}\left(\operatorname{Gr}_{G}\right), \mathcal{A} \in \mathcal{C}_{\kappa}^{G}(B) \mapsto \mathcal{F} \star \mathcal{A},
$$

where $\star$ denotes the convolution functor [30, which is well-defined because $\mathcal{A}$ is a $(\widehat{\mathfrak{g}}, G[[z]])$ Harish-Chandra module under the previous assumption (if $\kappa \neq \kappa^{\prime}$, we tensor $\mathcal{F}$ with an appropriate line bundle on $\mathrm{Gr}_{G}$ to make it into a $D_{\kappa^{\prime}}$-module before applying the convolution functor). Note that formula (6.7) coincides with formula 3.11 in the case $B=N_{1,0}^{G}$.

According to assumption (ii), we have a localization functor

$$
\Delta_{\kappa^{\prime}}: V_{\kappa}^{G}\left(D_{0,1}^{G} \rightarrow B\right)-\bmod \rightarrow D_{\kappa^{\prime}}\left(\operatorname{Bun}_{G}\right) .
$$

Denote its restriction to the subcategory $\mathcal{C}_{\kappa}^{G}(B)$ of $V_{\kappa}^{G}\left(D_{0,1}^{G} \rightarrow B\right)$ - $\bmod$ by $\left.\Delta_{\kappa^{\prime}}\right|_{\mathcal{C}_{\kappa}^{G}(B)}$.

Under the above assumptions, we now define the compactification functor $F_{\kappa}^{G, B}$ from $\mathcal{C}_{\kappa}^{G}(B)$ to $D_{\kappa}\left(\operatorname{Bun}_{G}\right)$ in terms of the localization functor $\left.\Delta_{\kappa^{\prime}}\right|_{\mathcal{C}_{\kappa}^{G}(B)}$. Namely, we set

$$
F_{\kappa}^{G, B}=\left.\Delta_{\kappa^{\prime}}\right|_{\mathcal{C}_{\kappa}^{G}(B)} \otimes \mathcal{L}_{G}^{\otimes m},
$$

where $\mathcal{L}_{G}$ is the minimal line bundle on $\operatorname{Bun}_{G}$ and $m \cdot n(G)=\kappa-\kappa^{\prime}$ (recall that tensoring with $\mathcal{L}_{G}$ shifts the level by $\left.n(G)\right)$.

To motivate the definition (6.8), let us compare the fibers of the twisted $D$-modules on the left and right hand sides of (6.8) for an object $\mathcal{A}$ of $\mathcal{C}_{\kappa}^{G}(B)$. On the left hand side, the fiber is

$$
\operatorname{Hom}\left(\delta_{\mathcal{P}}, F_{\kappa}^{G, B}(\mathcal{A})\right) .
$$

On the right hand side it is isomorphic to the space of $\mathcal{P}$-twisted coinvariants

$$
H_{V_{\kappa}^{G}\left(D_{0,1}^{G} \rightarrow B\right)}^{\mathcal{P}}(X, x, \mathcal{A}) .
$$

In order to show that these two spaces should indeed be isomorphic under our assumptions (i)-(iii), consider formula (6.7) in the case that $\mathcal{F}=\delta_{p}$, the $\delta$-function twisted $D$-module corresponding to a point $p \in \operatorname{Gr}_{G}=G((z)) / G[[z]]$. Let $\widetilde{p}$ be a lift of $p$ to $G((z))$. Then for any $(\widehat{\mathfrak{g}}, G[[z]])$ Harish-Chandra module $M$, we have

$$
\delta_{p} \star M \simeq \widetilde{p}^{*}(M) .
$$

where for a $\widehat{\mathfrak{g}}$-module $M$ and an element $h \in G((z))$, we denote by $h^{*}(M)$ the $\widehat{\mathfrak{g}}$-module on which the action of $\widehat{\mathfrak{g}}$ is modified by $\mathrm{Ad}_{h}$. Note that if the action of the Lie subalgebra $\mathfrak{g}[[z]] \subset \widehat{\mathfrak{g}}$ on $M$ extends to an action of the corresponding group $G[[z]]$, then $h_{1}^{*}(M) \simeq h_{2}^{*}(M)$ if the images of $h_{1}$ and $h_{2}$ in $\mathrm{Gr}_{G}$ coincide. That's why the right hand side of $(6.9)$ does not depend on the lift of $p$ to $G((z))$.

By our assumptions (ii) and (iii), the right hand side of $(6.9)$ describes the image of $\delta_{p} \otimes M$ under the functor 6.6 .

Recall that if $G$ is simple, then $\operatorname{Bun}_{G} \simeq G(X \backslash x) \backslash G((z)) / G[[z]]$. Let $p$ be a lift of $\mathcal{P} \in \operatorname{Bun}_{G}$ to $\operatorname{Gr}_{G}=G((z)) / G[[z]]$, and $\widetilde{p}$ a lift of $p$ to $G((z))$. We can think of $\widetilde{p}$ as a transition function on the punctured disc around $x$. If we glue the trivial bundles on the disc and on $X \backslash x$ using this transition function, we obtain the $G$-bundle $\mathcal{P}$ on $X$. (As explained in Section 
3.2 the compactification functor $F_{\kappa}^{G, D_{0,1}}$ sends $\delta_{p}, p \in \mathrm{Gr}_{G}$, to $\delta_{\mathcal{P}}$, where $\mathcal{P}$ is the image of $p$ in $\operatorname{Bun}_{G}=G(X \backslash x) \backslash \mathrm{Gr}_{G}$. $)$

The definition of twisted coinvariants readily implies that the space of $\mathcal{P}$-twisted coinvariants of $\mathcal{A}$ is the same as the space of untwisted coinvariants of $\widetilde{p}^{*}(\mathcal{A})$, which is $\delta_{p} \star \mathcal{A}$ by formula 6.9 . Hence

$$
H_{V_{\kappa}^{G}\left(D_{0,1}^{G} \rightarrow B\right)}^{\mathcal{P}}(X, x, \mathcal{A}) \simeq H_{V_{\kappa}^{G}\left(D_{0,1}^{G} \rightarrow B\right)}\left(X, x, \delta_{p} \star \mathcal{A}\right) .
$$

Now, according to assumption (ii) and formula (6.7), Conjecture 3.1 states that we have an isomorphism

$$
H_{V_{\kappa}^{G}\left(D_{0,1}^{G} \rightarrow B\right)}\left(X, x, \delta_{p} \star \mathcal{A}\right) \simeq \operatorname{Hom}\left(\delta_{\mathcal{P}}, F_{\kappa}^{G, B}(\mathcal{A})\right) .
$$

Combining the isomorphisms 6.10 and 6.11 , we find that the fibers at $\mathcal{P}$ of the two sides of (6.8) should indeed be isomorphic:

$$
\operatorname{Hom}\left(\delta_{\mathcal{P}}, F_{\kappa}^{G, B}(\mathcal{A})\right) \simeq H_{V_{\kappa}^{G}\left(D_{0,1}^{G} \rightarrow B\right)}^{\mathcal{P}}(X, x, \mathcal{A}) .
$$

Thus, the fibers of the two sides of $(6.8)$ are indeed isomorphic.

Since $\Delta_{\kappa^{\prime}}(\mathcal{A})$ is a $\kappa^{\prime}$-twisted $D$-module on $\operatorname{Bun}_{G}$, and $F_{\kappa}^{G, B}(\mathcal{A})$ is a $\kappa$-twisted $D$-module, in light of (6.12), this naturally leads us to stipulate that

$$
F_{\kappa}^{G, B}(\mathcal{A})=\Delta_{\kappa^{\prime}}(\mathcal{A}) \otimes \mathcal{L}_{G}^{\otimes m},
$$

where $m=\left(\kappa-\kappa^{\prime}\right) / n(G) \in \mathbb{Z}$. This motivates the definition (6.8).

Since there could be multiple junctions $D_{0,1}^{G} \rightarrow B$ with different vertex algebras associated to them, for this definition to be correct, we need the following:

Conjecture 6.1. For any two junctions $D_{0,1}^{G} \rightarrow B$ such that the corresponding functors (6.6) satisfy the assumptions (i)-(iii), the functors $\left.\Delta_{\kappa^{\prime}}\right|_{\mathcal{E}_{\kappa}^{G}(B)}$ are naturally isomorphic (after taking a tensor product with a power of $\mathcal{L}_{G}$ if the levels $\kappa^{\prime}$ corresponding to the two junctions are different).

This conjecture can be viewed as a generalization of Conjecture 2.2, according to which the space of coinvariants $H_{V_{\kappa}^{G}\left(D_{0,1}^{G} \rightarrow B\right)}^{\mathcal{P}}(X, x, \mathcal{A})$ depends (up to an isomorphism) only on $B$ and the object $\mathcal{A}$ of the category $\mathcal{C}_{\kappa}^{G}(B)$ (as well as $\mathcal{P} \in \operatorname{Bun}_{G}$ ), but is independent of the junction data between $D_{0,1}^{G}$ and $B$ used in the construction of the vertex algebra $V_{\kappa}^{G}\left(D_{0,1}^{G} \rightarrow B\right)$. Conjecture 6.1 extends this to an isomorphism of the corresponding twisted $D$-modules on $\operatorname{Bun}_{G}$.

Remark 6.2. The isomorphism (6.10), translated into the language of $4 \mathrm{~d}$ gauge theory, means that we can obtain the space of states $\mathbf{H}_{\mathcal{P}}^{B}(\mathcal{A})$ in the strip geometry discussed in Section 6.4 in two ways: the first is by coupling the Dirichlet boundary condition to a $G$ bundle $\mathcal{P}$ on $X$; and the second is by adding the line defect corresponding to $\delta_{p}$, where $p$ is a lifting of $\mathcal{P} \in \operatorname{Bun}_{G}$ to $\mathrm{Gr}_{G}$, at the boundary. This makes perfect sense because this line defect has the effect of changing the trivial $G$-bundle on $X$ to a bundle obtained by gluing the trivial $G$-bundles on the disc around $x$ and $X \backslash x$ using as the transition function, an element $\widetilde{p}$ of $G((z))$ defined as above. 
However, there is a subtle but important difference between these two procedures of obtaining the space $\mathbf{H}_{\mathcal{P}}^{B}(\mathcal{A})$ : When we obtain it by coupling the Dirichlet boundary condition to the $G$-bundle $\mathcal{P}$, as described in Section 6.4 , we automatically obtain a natural action on $\mathbf{H}_{\mathcal{P}}(\mathcal{A})$ of the group $\operatorname{Aut}(\mathcal{P})$ of automorphisms of the $G$-bundle $\mathcal{P}$ on $X$. On the other hand, if we instead insert the line defect $\delta_{p}$, we obtain the same vector space, but we forget the action of $\operatorname{Aut}(\mathcal{P})$. If we want these spaces to combine into a twisted $D$-module on $\operatorname{Bun}_{G}$, however, the information about the action of $\operatorname{Aut}(\mathcal{P})$ must be included, so we have to use the first procedure.

Likewise, the group $\operatorname{Aut}(\mathcal{P})$ acts naturally on the space $H_{V_{\kappa}^{G}\left(D_{0,1}^{G} \rightarrow B\right)}^{\mathcal{P}}(X, x, \mathcal{A})$ of $\mathcal{P}$-twisted coinvariants (which appears in our definition of the compactification functor as the localization functor) but doesn't acts on the space $H_{V_{\kappa}^{G}\left(D_{0,1}^{G} \rightarrow B\right)}\left(X, x, \delta_{p} \star \mathcal{A}\right)$.

Remark 6.3. Roughly speaking, the reason we are able to assign twisted $D$-modules on $\operatorname{Bun}_{G}$ to line defects associated to a boundary condition $B$ is that there is a family of line defects associated to the Dirichlet boundary condition parametrized by points of $\operatorname{Bun}_{G}$. What about the Neumann and Nahm boundary conditions? At first glance, the above construction can't be generalized to them because their standard line defects are parametrized by discrete data (integral weights or coweights of $G$ ). However, we actually do have a continuous parameter for those line defects as well; namely, the point $x$ of the Riemann surface $X$ at which we insert a junction from one of these boundary conditions to $B$. Unlike the Dirichlet case, for which varying $x$ does not change the compactification functor, in the Nahm and Neumann cases it does change. Therefore, using a variant of the above arguments, we obtain $D$-modules on $X$. More generally, by considering multiple points, we can obtain $D$-modules on the spaces of $P^{+}$- or ${ }^{L} P^{+}$-valued divisors on $X$. These $D$-modules can probably be glued together into $D$-modules on the corresponding Ran spaces.

6.6. A simple test. Consider the vertex algebra associated to the junction

$$
N_{1,0}^{G} \rightarrow D_{0,1}^{G}=R\left(D_{0,1}^{G} \rightarrow N_{1,0}^{G}\right)
$$

in the bulk theory $\mathcal{T}_{-\kappa}^{G}$. According to formula 3.10 , this vertex algebra is $V_{\kappa}(\widehat{\mathfrak{g}})$. Let us take the identity object in the category of line defects $\mathcal{C}_{-\kappa}^{G}\left(N_{1,0}^{G}\right)$ and the $\delta$-function $D$-modules $\delta_{p}, p \in \operatorname{Gr}_{G}$ in $\mathcal{C}_{-\kappa}^{G}\left(D_{0,1}^{G}\right)$. Applying to them the compactification functors, we obtain the sheaf of $\kappa$-twisted differential operators $\mathcal{D}_{\kappa}$ and the $\delta$-function $D$-modules $\delta_{\mathcal{P}}$ on $\operatorname{Bun}_{G}$. According to Conjecture 3.1 and the discussion of Section 6.5, we expect an isomorphism

$$
H^{\mathcal{P}}\left(X, x, V_{\kappa}(\mathfrak{g})\right) \simeq \operatorname{Hom}_{\mathcal{D}_{\kappa}}\left(\mathcal{D}_{\kappa}, \delta_{\mathcal{P}}\right) .
$$

To show that this isomorphism indeed holds, observe that the right hand side is nothing but the space of sections of $\delta_{\mathcal{P}}$, which we will denote by $\Gamma\left(\delta_{\mathcal{P}}\right)$. On the other hand, $H_{\mathcal{P}}\left(X, x, V_{k}(\mathfrak{g})\right)$ is also isomorphic to $\Gamma\left(\delta_{\mathcal{P}}\right)$. The proof of this fact (see, e.g., [28], Ch. 18) is straightforward: the tangent space to $\operatorname{Bun}_{G}$ at $\mathcal{P}$ is isomorphic to

$$
T_{\mathcal{P}} \operatorname{Bun}_{G} \simeq \mathfrak{g}_{\text {out }}^{\mathcal{P}} \backslash \mathfrak{g}((z)) / \mathfrak{g}[[z]],
$$

where $z$ is a local coordinate at $x \in X$. By definition,

$$
V_{\kappa}(\mathfrak{g})=U_{\kappa}(\widehat{\mathfrak{g}}) / U_{\kappa}(\widehat{\mathfrak{g}}) \cdot \mathfrak{g}[[z]],
$$


and $H_{\mathcal{P}}\left(X, x, V_{\kappa}(\mathfrak{g})\right)$ is the quotient of $V_{\kappa}(\mathfrak{g})$ by the action of the Lie subalgebra $\mathfrak{g}_{\text {out }}^{\mathcal{P}}$. This gives us an isomorphism

$$
H_{\mathcal{P}}\left(X, x, V_{k}(\mathfrak{g})\right) \simeq \Gamma\left(\delta_{\mathcal{P}}\right)
$$

which implies (6.13).

Finally, we can relate the right hand side of $(6.13)$ to a Hom of branes:

$$
\operatorname{Hom}_{\mathcal{D}_{\kappa}}\left(\mathcal{D}_{\kappa}, \delta_{\mathcal{P}}\right) \simeq \operatorname{Hom}\left(\mathcal{B}_{\text {c.c. }}, \mathcal{F}_{\mathcal{P}}^{\prime}\right) \text {. }
$$

Therefore we obtain

$$
H^{\mathcal{P}}\left(X, x, V_{\kappa}(\mathfrak{g})\right) \simeq \operatorname{Hom}\left(\mathcal{B}_{\text {c.c. }}, \mathcal{F}_{\mathcal{P}}^{\prime}\right)
$$

This isomorphism was discussed in [35, and an isomorphism between "tempered" versions of these vector spaces has been studied in [9] (note that in [9] this term refers to vectors in these spaces, and so it has no relation to the notion of tempered $D$-modules discussed in Remark 6.1).

6.7. Branes and Hecke eigensheaves. Let $\kappa=0$, corresponding to the critical level of $\widehat{\mathfrak{g}}$. In this case, Kapustin and Witten defined the $(B, A, A)$ branes $\left(\mathbf{F}_{b}, \nabla\right)$ supported on the fibers $\mathbf{F}_{b}$ of the Hitchin fibration in $\mathcal{M}_{H}(G)$ (see item (ii) on the list at the beginning of Section 6.1). They are mirror dual to the $(B, B, B)$ zero-branes $\mathbf{B}_{\varepsilon}$ supported at generic points of $\mathcal{M}_{H}\left({ }^{L} G\right)$ (in the complex structure $J$ on $\mathcal{M}_{H}\left({ }^{L} G\right)$ these are flat ${ }^{L} G$-bundles on $X$ that have no automorphisms other then those coming from the center of ${ }^{L} G$ ). Since $\mathbf{B}_{\varepsilon}$ is an eigenbrane of the Wilson operators, we obtain that the branes $\left(\mathbf{F}_{b}, \nabla\right)$ are eigenbranes of the 't Hooft operators (these are line defect operators corresponding to lines in the bulk of the $4 \mathrm{~d}$ gauge theory).

As we discussed in Section 6.1, the branes $\left(\mathbf{F}_{b}, \nabla\right)$ do not deform away from $\kappa=0$. However, at $\kappa=0$ they give rise to twisted $D_{0}$-modules on $\mathrm{Bun}_{G}$, which are Hecke eigensheaves, objects of interest in the geometric Langlands correspondence. (Recall that $D_{0}$-modules are $D$-modules twisted by a square root of the canonical line bundle, so a careful treatment of the corresponding category includes spin subtleties discussed in the later sections. But here we choose to ignore these subtleties.)

Based on gauge theory considerations [36, 37, 15, one can argue that the Hecke eigensheaves should also computable as conformal blocks of certain "kernel" vertex algebras, which have both a Kac-Moody subalgebra of level that is a multiple of $n(G)$ and an extra structure which allows the conformal blocks/coinvariants to be twisted by a flat ${ }^{L} G$-bundle.

These $\kappa=0$ kernel vertex algebras can sometimes be built directly from a microscopic description of the dual to Dirichlet boundary conditions, involving the three-dimensional SCFT called $T[G]$ in [41]. The description is only somewhat explicit for some classical gauge groups and it cannot be deformed to irrational $\kappa$.

Still, somewhat experimentally, it appears to lead to kernel vertex algebras $V(T[G])$ which are, in an appropriate sense the $\kappa \rightarrow 0$ limits of $V_{\kappa}^{G}\left(D_{0,1}^{G} \rightarrow D_{1,0}^{G}\right)$ : up to some rescalings, the OPE of $V_{\kappa}^{G}\left(D_{0,1}^{G} \rightarrow D_{1,0}^{G}\right)$ has a finite limit which coincides with $V(T[G])$, with the level ${ }^{L} \kappa$ Kac-Moody currents for ${ }^{L} G$ replaced in the limit ${ }^{L} \kappa \rightarrow \infty$ by a classical holomorphic connection for ${ }^{L} G$. This allows conformal blocks of $V(T[G])$ to be twisted by a flat ${ }^{L} G$-bundle. Conjecturally, $V(T[G])$ can be obtained in this fashion even when $T[G]$ is not directly known. 
More generally, vertex algebras of the form $V_{\kappa}^{G}\left(D_{1,0}^{G} \rightarrow B\right)$ tend to have a very nice $\kappa \rightarrow 0$ behaviour [36, 37, 16, 15]: up to some rescalings the OPE have a finite limit giving rise to a vertex algebra $V_{0}^{G}(B)$, with the level ${ }^{L_{\kappa}}$ of Kac-Moody currents for ${ }^{L} G$ replaced by a classical holomorphic connection for ${ }^{L} G$. Again, this allows conformal blocks of $V_{0}^{G}(B)$ to be twisted by a flat ${ }^{L} G$-bundle. We expect the $D$-module of coinvariants of $V_{0}^{G}(B)$ to be the Geometric Langland dual of the $D$-module of coinvariants of $V_{0}^{G}\left(D_{0,1}^{G} \rightarrow B\right)$. We hope to explore this in more detail in a future work.

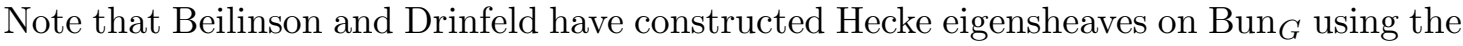
isomorphism of [22, 24]. In this subsection we discuss a link between these two constructions in the framework of our general formalism.

Recall that according to [22, 24], the vacuum module $V_{0}(\mathfrak{g})$ of $\widehat{\mathfrak{g}}$ of critical level has a large algebra of endomorphisms, which is isomorphic to the algebra of functions on the space $\operatorname{Op}_{L_{G}}\left(D_{x}\right)$ of opers for the Langlands dual group ${ }^{L} G$ on the formal disc $D_{x}$ at $x$. For $\rho \in \mathrm{Op}_{L_{G}}\left(D_{x}\right)$, we can then define a $\widehat{\mathfrak{g}}$-module $\mathbb{V}_{\rho}$ of critical level as the quotient of $V_{0}$ by the ideal $I_{\rho}$ corresponding to $\rho$ :

$$
V_{\rho}=V_{0} / I_{\rho}
$$

In other words, we set all central elements of the enveloping algebra of $\widehat{\mathfrak{g}}$ (such as the quadratic Sugawara elements) equal to the numeric values prescribed by $\rho$ (see [25] for more details).

Now suppose that $x$ is a point of a Riemann surface $X$ (and so $D_{x} \subset X$ ). The following theorem is due to Beilinson and Drinfeld [11].

\section{Theorem 6.2.}

(1) $\mathcal{H}^{\mathcal{P}}\left(X, x, V_{\rho}\right)=0$ for all $\mathcal{P} \in \operatorname{Bun}_{G}$, unless the ${ }^{L} G$-oper $\rho$ extends from the disc $D_{x}$ to the entire curve $X$.

(2) If $\rho$ does extend to an ${ }^{L} G$-oper on $X$, then the critically twisted D-module on $\operatorname{Bun}_{G}$ $\Delta_{0}\left(V_{\rho}\right)$, whose stalks are the spaces of coinvariants $\mathcal{H}^{\mathcal{P}}\left(X, x, V_{\rho}\right)$ is a Hecke eigensheaf whose eigenvalue is the flat ${ }^{L} G$-bundle on $X$ defined by this oper.

The two perspectives on Hecke eigensheaves will agree if $\mathcal{H}^{\mathcal{P}}\left(X, x, \mathbf{V}_{\rho}\right)$ coincides with the space of coinvariants for $V(T[G])$ twisted by a flat ${ }^{L} G$-bundle which is also an oper. In concrete examples this seems to happen in a very direct fashion: coupling to an oper deforms $V(T[G])$ to a vertex algebra equivalent to $V_{\rho}$.

For completeness, we now recall the definition of the Kapustin-Witten $(B, A, A)$ branes on $\mathcal{M}_{H}(G)$ that are dual to the zero-branes $\mathbf{B}_{\mathcal{E}}$ on $\mathcal{M}_{H}\left({ }^{L} G\right)$ [55]. Recall that here $\mathcal{E}$ is a flat ${ }^{L} G$-bundles on $X$ that have no automorphisms other then those coming from the center of ${ }^{L} G$, viewed as a point in the Hitchin moduli space $\mathcal{M}_{H}\left({ }^{L} G\right)$ with respect to the complex structure $J$. Now consider $\mathcal{E}$ as a point of $\mathcal{M}_{H}\left({ }^{L} G\right)$ with respect to the complex structure $I$, and let $b=b(\mathcal{E})$ be its image in the Hitchin base under the (first) Hitchin fibration. Let $\mathbf{F}_{b}$ be the dual fiber in $\mathcal{M}_{H}(G)$. Then the mirror dual brane to $\mathbf{B}_{\varepsilon}$ is the pair $\left(\mathbf{F}_{b}, \nabla\right)$, where $\nabla=\nabla_{\mathcal{E}}$ is a flat unitary line bundle on $\mathbf{F}_{b}$ corresponding to $\mathcal{E}$ under the $T$-duality of Hitchin fibers in $\mathcal{M}_{H}(G)$ and $\mathcal{M}_{H}\left({ }^{L} G\right)$.

Suppose that $\mathcal{E}=\mathcal{E}(\rho)$ corresponds to an oper $\rho$. Then we find from formula 6.5 that there should be an isomorphism between the space of $\mathcal{P}$-twisted conformal blocks of the 
$\widehat{\mathfrak{g}}$-module $\mathbf{V}_{\rho}$ and a Hom in the category of $A$-branes on $\mathcal{M}_{H}(G)$ (with respect to $\omega_{K}$ ):

$$
\mathcal{H}^{\mathcal{P}}\left(X, x, \mathbf{V}_{\rho}\right) \simeq \operatorname{Hom}\left(\mathcal{F}_{\mathcal{P}},\left(\mathbf{F}_{b}, \nabla_{\varepsilon}\right)\right) .
$$

This isomorphism has been previously discussed in [35, 9].

As Balasubramanian and Teschner argued in 9], one can think of this isomorphism as an expression of a link between the Beilinson-Drinfeld construction and the Kapustin-Witten construction of the Geometric Langlands correspondence. Indeed, it shows how to express the fibers of the Hecke eigensheaf, i.e. a $D_{0}$-module on $\mathrm{Bun}_{G}$ obtained by applying the localization functor $\Delta_{0}$ to $\mathbf{V}_{\rho}$ in terms of Hom's between the fiber-brane $\mathcal{F}_{\mathcal{P}}$ discussed in Section 6.1 and the Kapustin-Witten brane $\left(\mathbf{F}_{b}, \nabla_{\varepsilon}\right)$.

As we explained in [36] and Section 6.3 above, the spaces appearing on the right hand side of (6.17) acquire a natural flat (Berry) connection, which should coincide with the one on the left hand side, coming from the $D$-module structure on $\Delta_{0}\left(\mathbf{V}_{\rho}\right)$.

As a simple test of (6.17), we can check that for a generic $G$-bundle $\mathcal{P}$, the dimensions of the two vector spaces in the isomorphism (6.17) are the same. According to the left hand side, this is the generic rank of the $D$-module $\Delta_{0}\left(\mathbf{V}_{\rho}\right)$, which is the multiplicity of the nilpotent cone in the zero fiber of the (first) Hitchin fibration. It coincides with the number of points in the intersection of generic fibers $\mathbf{F}_{b}$ and $\mathcal{F}_{\mathcal{P}}$ of the first and second Hitchin fibrations. But the latter is the dimension of the generic space on the right hand side of 6.17).

\section{A simple example: $U(1)$ gauge theory}

The simplest Abelian example - the $4 \mathrm{~d} N=4$ supersymmetric $U(1)$ gauge theory is already rich enough to demonstrate non-trivial compositions of junctions. It is also an excellent example of the potential spin structure dependence of our constructions and of the extra subtleties which occur when the spin structure dependence is lifted.

The notions of Nahm and Dirichlet boundary conditions coincide in Abelian theories, so we have a single family of boundary conditions $N_{p, q}^{U(1)}$, which we will denote in this subsection simply by $N_{p, q}$.

7.1. Category of boundary lines. The tensor category $\mathcal{C}_{\kappa}^{G}\left(N_{1,0}\right)$ of line defects associated to the boundary condition $N_{1,0}$ is $\mathrm{KL}_{\kappa}(U(1)){ }^{17}$ It is a semisimple category with irreducible objects $L_{n}$ for integer $n$, and the tensor product (fusion) $L_{n} \otimes L_{m} \simeq L_{n+m}$. The twisting (aka topological spin) on $L_{n}$ is given by $e^{\pi i \frac{n^{2}}{\kappa}}$. We will call its phase factor $\pi \frac{n^{2}}{\kappa}$ the twist.

For now, we will consider $\mathcal{C}_{\kappa}^{G}\left(N_{1,0}\right)$ as what we will call a "spin-ribbon category." This is a modification of the ribbon category in which the twistings are defined up to multiplication by \pm 1 . In other words, the twists are only defined up to addition of an integer multiple of $\pi$ (rather than $2 \pi)$. The necessity to view $\mathcal{C}^{G} \kappa\left(N_{1,0}\right)$ as a spin-ribbon category can be inferred, for example, from the fact that some of the dualities from the group $S L_{2}(\mathbb{Z})$ preserve the spin-ribbon structure but not the ribbon structure.

\footnotetext{
${ }^{17}$ If $G_{c}$ is a compact Lie group and $\mathfrak{g}_{c}$ its Lie algebra, we will sometimes refer to the affine Kac-Moody algebra $\widehat{\mathfrak{g}}$, where $\mathfrak{g}$ is the complexification of $\mathfrak{g}_{c}$, as the $\mathfrak{g}_{c}$ (affine) Kac-Moody algebra, and use $\mathfrak{g}_{c}$ rather than $\mathfrak{g}$ in denoting various categories and functors associated to $\widehat{\mathfrak{g}}$. For example, we will sometimes use the notation $U(1)$ instead of $G L(1)$, e.g., $\mathrm{KL}_{\kappa}(U(1))$.
} 
For example, the action of STS maps $\kappa \mapsto\left(\kappa^{-1}-1\right)^{-1}$, and hence the twist $\pi \frac{n^{2}}{\kappa}$ gets mapped to $\pi n^{2}\left(\kappa^{-1}-1\right)$. The corresponding categories $\mathcal{C}_{\kappa}^{G}\left(N_{1,0}\right)$ and $\mathcal{C}_{\left(\kappa^{-1}-1\right)^{-1}}^{G}\left(N_{1,0}\right)$ would only be equivalent if we stipulate that the twists in them are defined up to addition of integer multiples of $\pi$.

7.2. Junction vertex algebras. The basic junction from $N_{0,1} \rightarrow N_{1,0}$ supports the $\widehat{\mathfrak{u}}(1)$ Kac-Moody algebra at level $\kappa$ (which is its own $\mathcal{W}$-algebra). The standard modules correspond to the $\widehat{\mathfrak{u}}(1)$ vertex operators of charge $n+m \kappa$ and conformal dimension

$$
\Delta_{m, n}^{\mathfrak{u}(1)}(\kappa)=\frac{n^{2}}{2 \kappa}+n m+\frac{m^{2} \kappa}{2} .
$$

They are the images of the corresponding objects of the category $\mathrm{KL}_{\kappa}(U(1)) \otimes \mathrm{KL}_{\kappa^{-1}}(U(1))$

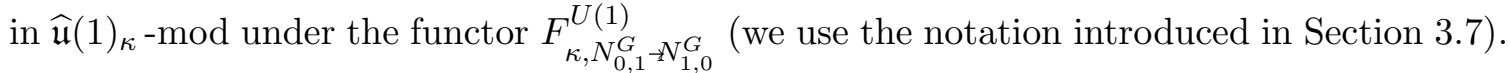

In the same way as in the last example of Section 3.7, we find that applying the duality transformation $R S T^{-1}$ to the standard junction $N_{0,1} \rightarrow N_{1,0}$ we obtain a junction $N_{1,-1} \rightarrow$ $N_{1,0}$ supporting the $\widehat{\mathfrak{u}}(1)$ Kac-Moody algebra at level $\kappa^{-1}+1$. On the other hand, applying $T^{-1}$ we obtain the junction $N_{0,1} \rightarrow N_{1,-1}$ supporting the $\widehat{\mathfrak{u}}(1)$ Kac-Moody algebra at level $\kappa+1$.

The composition of two such junctions, $N_{0,1} \rightarrow N_{1,-1} \rightarrow N_{1,0}$, gives an extension of $\widehat{\mathfrak{u}}(1)_{\kappa+1} \times \widehat{\mathfrak{u}}(1)_{\kappa^{-1}+1}$ Kac-Moody by new fields of dimensions

$$
\Delta_{0, n}^{\mathfrak{u}(1)}(\kappa+1)+\Delta_{n, 0}^{\mathfrak{u}(1)}\left(\frac{\kappa}{\kappa+1}\right)=\frac{n^{2}}{2} \frac{1}{\kappa+1}+\frac{n^{2}}{2} \frac{\kappa}{\kappa+1}=\frac{n^{2}}{2} .
$$

The extension can be identified with the product of a $\widehat{\mathfrak{u}}(1)$ Kac-Moody algebra at level $\kappa$ and of the lattice vertex superalgebra corresponding to the lattice $\mathbb{Z}$. The $\widehat{\mathfrak{u}}(1)$ current of the former is $\frac{\kappa}{\kappa+1}\left(J_{\kappa+1}-J_{\kappa^{-1}+1}\right)$ and the $\widehat{\mathfrak{u}}(1)$ current of the latter is $\frac{1}{\kappa+1} J_{\kappa+1}+\frac{1}{\kappa^{-1}+1} J_{\kappa^{-1}+1}$. The latter is isomorphic to the free (complex) fermion vertex superalgebra $\bigwedge$. It is generated by two fermionic fields of conformal dimensions $1 / 2$ which correspond to $n= \pm 1$ in formula (7.2).

More general modules over this vertex algebra combine vertex operators of dimension

$$
\Delta_{m, n}^{\mathfrak{u}(1)}(\kappa+1)+\Delta_{n, e}^{\mathfrak{u}(1)}\left(\frac{\kappa}{\kappa+1}\right)=\frac{(n+e+m)^{2}}{2}+\Delta_{m,-e}^{\mathfrak{u}(1)}(\kappa)
$$

Thus, the vertex algebra $V_{\kappa}^{U(1)}\left(N_{0,1} \rightarrow N_{1,0}\right)$ obtained from the composition of junctions $N_{0,1} \rightarrow N_{1,-1} \rightarrow N_{1,0}$ is the vertex algebra $V_{\kappa}^{U(1)}\left(N_{0,1} \rightarrow N_{1,0}\right)$ of the basic junction $N_{0,1} \rightarrow$ $N_{1,0}$ tensored with the free fermion vertex superalgebra. From the point of view of $4 \mathrm{~d}$ gauge theory, both vertex algebras are associated to the junction $N_{0,1} \rightarrow N_{1,0}$, but with different junction data 18

According to Conjecture 2.2, we expect the spaces of conformal blocks of the two vertex algebras to be isomorphic. And indeed, this is the case, because the space of conformal blocks of the free fermion vertex superalgebra $\bigwedge$ is one-dimensional for any compact Riemann surface $X$. However, because the two generating fields of $\bigwedge$ have conformal dimensions

\footnotetext{
${ }^{18}$ Physically, the second junction is obtained from the first by "stacking" it with a decoupled, holomorphic $2 \mathrm{~d}$ spin-CFT: a free complex fermion. In particular, the second junction is intrinsically a "spin junction".
} 
$1 / 2$, in order to define this space of conformal blocks we need to choose a square root $K^{1 / 2}$ of the canonical line bundle on $X$. This choice is equivalent to a choice of spin structure on $X$. This is another example of how spin structures show up in the twisted TFT.

We can also construct new junctions. For example, we can compose the basic junction $N_{0,1} \rightarrow N_{1,0}$ with the junction $N_{1,0} \rightarrow N_{k, 1}$ obtained as $S T^{-k}$ image of the basic junction $N_{0,1} \rightarrow N_{1,0}$. For now, take $k>0$.

The result is a junction $N_{0,1} \rightarrow N_{k, 1}$, which is not a duality image of the basic junction: there is no combination of $S$ and $T$ operations which could relate it to $N_{0,1} \rightarrow N_{1,0}$. The corresponding vertex algebra is an extension of $\widehat{\mathfrak{u}}(1)_{\kappa} \times \widehat{\mathfrak{u}}(1)_{k-\kappa^{-1}}$ by operators of conformal dimension $\frac{k}{2} n^{2}$. It is isomorphic to the product of the $\widehat{\mathfrak{u}}(1)_{\kappa-k^{-1}}$ Kac-Moody algebra and the $u(1)_{k}$ lattice vertex algebra corresponding to the lattice $\sqrt{k} \mathbb{Z}$.

More general modules have dimensions

$$
\Delta_{m, n}^{\mathfrak{u}(1)}(\kappa)+\Delta_{n, e}^{\mathfrak{u}(1)}\left(k-\kappa^{-1}\right)=\frac{(k n+e+m)^{2}}{2 k}+\frac{m^{2}}{2 k}(k \kappa-1)+\frac{e^{2}}{2 k} \frac{1}{k \kappa-1}-\frac{e m}{k}
$$

and combine modules over $u(1)_{k}$ with modules over $\widehat{\mathfrak{u}}(1)_{\kappa-k^{-1}}$ Kac-Moody algebra with integral magnetic charges (corresponding to $m$ ) but electric charges of the form $e / k, e \in \mathbb{Z}$.

For $k=0$, the extension involves modules of conformal dimension 0 and is isomorphic to the vertex algebra of chiral differential operators on $G L_{1}(\mathbb{C})=\mathbb{C}^{\times}$. Indeed, by rescaling the first and the second factors of the $\widehat{\mathfrak{u}}(1)_{\kappa} \times \widehat{\mathfrak{u}}(1)_{-\kappa^{-1}}$ current algebra by $\kappa^{-1 / 2}$ and $\kappa^{1 / 2}$, respectively, we obtain the $\widehat{\mathfrak{u}}(1)_{1} \times \widehat{\mathfrak{u}}(1)_{-1}$ current algebra of the standard $\beta \gamma$ system in which we make the field $\gamma$ invertible. Note that under this identification, the commuting $\widehat{\mathfrak{u}}(1)$ currents are constructed from the fields $\beta \gamma$ and $\gamma^{-1} \partial \gamma$, and the extension vertex operators correspond to $\gamma^{n}, n \in \mathbb{Z}$.

For $k<0$, the extension involves modules of dimension unbounded from below and it is less well-behaved as a vertex algebra.

More generally, we would like to construct junctions $N_{0,1} \rightarrow N_{p, q}$ such that in the corresponding vertex algebra all fields, except the vacuum, have positive conformal dimensions. This is a favorable property for many reasons, not least the behavior of conformal blocks.

To achieve that, we need a chain of junctions which support $\widehat{\mathfrak{u}}(1)_{\kappa_{i}}, i=0, \ldots, n$, with

$$
\frac{1}{\kappa_{i-1}}+\kappa_{i}=k_{i}
$$

where each $k_{i}$ is a positive integer.

Such $\widehat{\mathfrak{u}}(1)_{\kappa_{i}}$ can be found at the junction $N_{0,1} \rightarrow N_{1,0}$ when the bulk coupling is $\kappa_{i}$.

Applying $S T^{-k_{i}}$, we find that it can also be found at the junction $N_{1,0} \rightarrow N_{k_{i}, 1}$ when the bulk coupling is

$$
-\frac{1}{\kappa_{i}-k_{i}}=\kappa_{i-1}
$$

We can then compose the $N_{0,1} \rightarrow N_{1,0}$ junction and the $N_{1,0} \rightarrow N_{k_{i}, 1}$ junction with the bulk coupling $\kappa_{i-1}$ into a junction $N_{0,1} \rightarrow N_{k_{i}, 1}$.

Note that the same vertex algebra will appear when the bulk coupling is $\kappa_{i-2}=-1 /\left(\kappa_{i-1}-\right.$ $\left.k_{i-1}\right)$ at the composition $N_{1,0} \rightarrow N_{k_{i-1}, 1} \rightarrow N_{k_{i} k_{i-1}-1, k_{i}}$. So, we can compose it with $N_{0,1} \rightarrow N_{1,0}$ to get a junction $N_{0,1} \rightarrow N_{k_{i} k_{i-1}-1, k_{i}}$. 
Next, move to the bulk coupling $\kappa_{i-3}=-1 /\left(\kappa_{i-2}-k_{i-2}\right)$ by applying $S T^{-k_{i-2}}$ and compose to get $N_{0,1} \rightarrow N_{k_{i} k_{i-1} k_{i-2}-k_{i}-k_{i-2}, k_{i} k_{i-1}-1}$.

If we run this procedure backwards from $\kappa_{n}$ to $\kappa_{0}$, we get junctions of the form

$$
\begin{aligned}
N_{0,1} \rightarrow N_{1,0} \rightarrow S T^{-k_{1}} & \left(N_{1,0}\right) \rightarrow S T^{-k_{1}} S T^{-k_{2}}\left(N_{1,0}\right) \rightarrow \\
& S T^{-k_{1}} S T^{-k_{2}} S T^{-k_{3}}\left(N_{1,0}\right) \rightarrow \cdots \rightarrow S T^{-k_{1}} S T^{-k_{2}} \ldots S T^{-k_{n}}\left(N_{1,0}\right)
\end{aligned}
$$

with $k_{i}>0$, i.e.,

$$
N_{0,1} \rightarrow N_{1,0} \rightarrow N_{k_{1}, 1} \rightarrow N_{k_{1} k_{2}-1, k_{2}} \rightarrow N_{k_{1} k_{2} k_{3}-k_{1}-k_{3}, k_{2} k_{3}-1} \rightarrow \cdots
$$

If we label the $i$ th term after $N_{1,0}$ by $N_{p_{i}, q_{i}}$ then

$$
\frac{p_{i}}{q_{i}}=k_{1}-\frac{1}{k_{2}-} \frac{1}{k_{3}-} \cdots \frac{1}{k_{i}}
$$

can be seen as the $i$ th convergent (i.e., the truncation after the first $i$ terms) of the continued fraction

$$
\frac{p_{i}}{q_{i}}=k_{1}-\frac{1}{k_{2}-} \frac{1}{k_{3}-} \cdots
$$

The junctions support $\widehat{\mathfrak{u}}(1)_{\kappa_{i}}$ Kac-Moody with $\kappa_{i}=k_{i}-\kappa_{i-1}^{-1}$ and $\kappa_{0}=\kappa$. The extension involves modules of charges $q_{i} \kappa_{i}+q_{i+1}$ for $\widehat{\mathfrak{u}}(1)_{\kappa_{i}}$ (with $q_{0}=0$ ) of dimension

$$
\sum_{i=1}^{n} \frac{k_{i}}{2} q_{i}^{2}+q_{i} q_{i+1}
$$

This vertex algebra is isomorphic to the product of a lattice vertex algebra of rank $n$ and the $\widehat{\mathfrak{u}}(1)$ Kac-Moody algebra at level

$$
\kappa_{p_{n}, q_{n}}=\kappa+\frac{1}{k_{1}-} \frac{1}{k_{2}-} \cdots \frac{1}{k_{n}}
$$

with the image of $\mathrm{KL}_{\kappa^{-1}}(U(1)) \times \mathrm{KL}_{\kappa_{n}}(U(1))$ given by combinations of modules for the lattice vertex algebra and modules of integral magnetic charges and fractional electric charges for the $\widehat{\mathfrak{u}}(1)$ Kac-Moody algebra.

All fields of this vertex algebra, except for the vacuum, have positive conformal dimensions.

This construction can serve as a prototype for building a vertex algebra satisfying this property corresponding to a junction from $N_{0,1}$ to any $N_{p, q}$ : expand $\frac{p}{q}$ into continued fractions and use the resulting integers $k_{i}$ to build the junction as a composition of basic junctions, as explained above.

7.3. Compactification functors. We can now discuss the compactification functors from the categories $\mathcal{C}_{\kappa}^{U(1)}(B)$ to $D_{\kappa}\left(\operatorname{Bun}_{U(1)}\right)$ (see Section 3.1.

We begin with two important points:

- There is a degree 1 line bundle $\mathcal{L}$ on $\operatorname{Bun}_{U(1)}$ related to the level 1 Chern-Simons action. It is the line bundle of conformal blocks for the $u(1)_{1}$ lattice vertex algebra (the vertex superalgebra $\bigwedge$ of a free complex fermion). Again, we are ignoring the spin structure for the moment. 
- The category $\mathcal{C}_{\kappa}^{U(1)}\left(N_{1,0}\right)$ is $\mathrm{KL}_{\kappa}(U(1))$, and the compactification functor $F_{\kappa}^{U(1), N_{1,0}}$ is the localization functor $\Delta_{\kappa}$. Applying it to the identity object of $\mathrm{KL}_{\kappa}(U(1))$, which is the vertex algebra $V_{\kappa}(U(1))$, we obtain the sheaf $\mathcal{D}_{\kappa}$ itself (see Section 6). This is a special case of the general statement discussed in example (ii) of Section 3.2 the identity object of $\mathcal{C}_{\kappa}^{U(1)}\left(N_{1,0}\right)$ is mapped to $\mathcal{D}_{\kappa}$. In other words, the compactification map sends the boundary condition $N_{1,0}$ to $\mathcal{D}_{\kappa}$.

The construction of a junction from $N_{0,1} \rightarrow N_{1,1}$ as a composition $N_{0,1} \rightarrow N_{1,0} \rightarrow N_{1,1}$ of the basic junction and the junction $N_{1,0} \rightarrow N_{1,1}$ discussed in the previous subsection (the case $k=1$ of the junction $\left.N_{1,0} \rightarrow N_{k, 1}\right)$ is instructive.

It produces a junction vertex algebra isomorphic to $u(1)_{1} \otimes V_{\kappa-1}(U(1))$, with the coupling to $U(1)$ bundles implemented by the diagonal combination of the $\widehat{\mathfrak{u}}(1)$ currents of the two factors. This junction vertex algebra yields a compactification functor whose image lies directly in $D_{\kappa}\left(\operatorname{Bun}_{U(1)}\right)$. The image of the compactification functor is the product of the sheaves of coinvariants of the two vertex subalgebras $u(1)_{1} \otimes V_{\kappa-1}(U(1))$, i.e. $\mathcal{L} \otimes \mathcal{D}_{\kappa-1}$. In other words, the compactification map sends the boundary condition $N_{1,1}$ to $\mathcal{L} \otimes \mathcal{D}_{\kappa-1}$.

We could also produce a $N_{0,1} \rightarrow N_{1,1}$ junction as a $T$ image of the $N_{0,1} \rightarrow N_{1,0}$ junction (up to the spin subtleties discussed in the next subsection). Since $N_{1,1}=T\left(N_{1,0}\right)$,

$$
\mathcal{C}_{\kappa}^{U(1)}\left(N_{1,1}\right) \simeq \mathcal{C}_{\kappa-1}^{U(1)}\left(N_{1,0}\right)=\mathrm{KL}_{\kappa-1}(U(1))
$$

Thus, the relevant localization functor for this second junction is $\Delta_{\kappa-1}$ taking values in $D_{\kappa-1}\left(\operatorname{Bun}_{U(1)}\right)$. When we apply it to the identity object of $\mathcal{C}_{\kappa}^{U(1)}\left(N_{1,1}\right)$ (i.e. the junction vertex algebra itself), we obtain $\mathcal{D}_{\kappa-1}$, viewed as a left $(\kappa-1)$-twisted $D$-module on $\operatorname{Bun}_{U(1)}$. As explained in Section 6.5, to get the corresponding compactification functor $F_{\kappa}^{G, N_{1,1}}$, we should tensor $\Delta_{\kappa-1}$ with the line bundle $\mathcal{L}$. Under $F_{\kappa}^{G, N_{1,1}}$, the identity object of $\mathcal{C}_{\kappa}^{U(1)}\left(N_{1,1}\right)$ therefore goes to $\mathcal{L} \otimes \mathcal{D}_{\kappa-1}$, in agreement with the previous construction.

This example provides a positive test for our prescription for the compactification functor. Is also demonstrates how the compactification functors intertwine the action of the duality $T$ on the categories corresponding to boundary conditions and the operation of taking a tensor product with $\mathcal{L}$, in agreement with Conjecture $4.1{ }^{19}$

Similar considerations apply to show that the compactification map sends $N_{1, k}$ to $\mathcal{L}^{k} \otimes$ $\mathcal{D}_{\kappa-k}$.

Next, we look at $N_{k, 1}$. As explained above, the vertex algebra corresponding to the junction $N_{0,1} \rightarrow N_{k, 1}$ is $u(1)_{k} \times \widehat{\mathfrak{u}}(1)_{\kappa-k^{-1}}$. If $|k|>1$, we cannot use the localization functor $\Delta_{\kappa-k^{-1}}$ corresponding to the $\widehat{\mathfrak{u}}(1)_{\kappa-k^{-1}}$ subalgebra because then $\kappa-k^{-1}$ differs from $\kappa$ by a rational number that is is not an integer. However, this junction vertex algebra has a diagonal $\widehat{\mathfrak{u}}(1)_{\kappa}$ subalgebra generated by the current which is the linear combination $k^{-1} J_{u(1)_{k}}+J_{\kappa-k^{-1}}$ of the generating currents of $u(1)_{k} \times \widehat{\mathfrak{u}}(1)_{\kappa-k^{-1}}$. The compactification

\footnotetext{
${ }^{19}$ From the point of view of $4 \mathrm{~d}$ gauge theory, this is clear from the fact that if we have a domain wall such that gauge theories on the two sides of the wall differ by the duality symmetry $T$ (i.e. have coupling constants $\kappa$ and $\kappa+1$ ), then the difference between the topological terms in the $4 \mathrm{~d}$ actions on the two sides (each of them is a scalar multiple of the first Pontryagin class, and the two scalars differ by $2 \pi$ ) has to be compensated on the boundary by the Chern-Simons action of level 1 , which is the $4 \mathrm{~d}$ gauge theory counterpart of tensoring with the line bundle $\mathcal{L}$ on $\operatorname{Bun}_{U(1)}$.
} 
functor $F_{\kappa}^{U(1), N_{k, 1}}$ can be obtained from the localization functor $\Delta_{\kappa}$ with respect to this subalgebra.

In particular, applying the localization functor $\Delta_{\kappa}$ to the identity object in $\mathcal{C}_{\kappa}^{U(1)}\left(N_{k, 1}\right)$, we obtain a $D_{\kappa}$-module on $\operatorname{Bun}_{U(1)}$ which is easy to describe as an $\mathcal{O}$-module: it is the tensor product of the bundles of coinvariants for $u(1)_{k}$ and $\widehat{\mathfrak{u}}(1)_{\kappa-k^{-1}}$, i.e. $\mathcal{V}_{k} \otimes \mathcal{D}_{\kappa-k^{-1}}$. Here $\mathcal{V}_{k}$ is the vector bundle of coinvariants for $u(1)_{k}$ which is coupled "magnetically" to the line bundles in $\operatorname{Bun}_{U(1)}$ in a non-trivial way: given a line bundle $\ell$ in $\operatorname{Bun}_{U(1)}$, we represent it as $\ell=\mathcal{O}(D)$, where $D=\sum_{i} n_{i} x_{i}$ is a divisor on $X$, and then take the space of coinvariants with the insertions of $u(1)_{k}$ primary fields of charge $n_{i}$ at the points $x_{i}$. (The statement that $\mathcal{V}_{k} \otimes \mathcal{D}_{\kappa-k^{-1}}$ has the structure of a $D_{\kappa}$-module is non-trivial. It follows from the fact that it is in the image of $\Delta_{\kappa}$.)

Thus, we have describe the image of $N_{k, 1}$ under the compactification map. More generally, by using the composition of junctions above, we can obtain the image of any $N_{p, q}$ as a twisted $D$-module of the form $\mathcal{L}^{[q / p]} \otimes \mathcal{V}_{p, q} \otimes \mathcal{D}_{\kappa_{p_{n}, q_{n}}-[q / p]}$ with $\mathcal{V}_{p, q}$ being the bundle of coinvariants of the lattice vertex algebra built above as an extension of $\otimes_{i} u(1)_{k_{i}}$.

It would be nice to prove directly that the qGL dualities $\mathcal{E}_{\kappa}^{U(1), g}$ intertwine these $D$ modules for $(p, q)$ and $g \circ(p, q)$. The simplest statement corresponds to the fact that $S\left(N_{1,-1}\right)=N_{1,1}$. This implies that the qGL duality $S$ sends

$$
S\left(\mathcal{L}^{-1} \otimes \mathcal{D}_{\kappa+1}\right) \mapsto \mathcal{L} \otimes \mathcal{D}_{-\kappa^{-1}-1},
$$

which is a special case of formula (1.7).

It is worth mentioning that all the $N_{p, q}$ boundary conditions can be given an interpretation as modifications of Neumann boundary conditions involving coupling to some specific 3d Chern-Simons theories, i.e. to some physical 3d TFTs. One may consider many more such boundary conditions, associated to more general $3 \mathrm{~d}$ TFTs which can be coupled to $U(1)$ connections.

7.4. Spin subtleties. Here we will discuss under which conditions we can dispense with the use of spin manifolds for the bulk theory and possibly for its boundary conditions and junctions.

Recall that the $4 \mathrm{~d}$ topological field theory $\mathcal{T}_{\kappa}^{G}$ we are interested in is obtained by a topological twist (the GL twist of [55]) from the physical supersymmetric gauge theory. This means that the action of the Lorentz group on the field content of the theory has been modified in such a way that the resulting twisted theory is endowed with a two-dimensional family of supercharges that can used to define a TFT (the physical theory is not endowed with supercharges). In particular, while in the physical theory the fermions transform as spinors, in the twisted TFT they are turned into $i$-forms with $i=0,1,2$. Thus, while the physical theory can only be defined on a spin four-manifold $M$ and one needs to make a specific choice of the spin structure to make the theory well-defined, the twisted TFT does not require $M$ to be a spin manifold, nor is one required to make a choice of the spin structure.

However, there is a price to pay: it turns out that the action of the quantum dualities then has to be refined. If we choose a spin structure, then every element of the group $P S L_{2}(\mathbb{Z})$ gives rise to a legitimate quantum duality of the theory $\mathcal{T}_{\kappa}^{U(1)}$ (though the categories of line 
defects are then spin-ribbon categories, as discussed above). However, if we do not have a spin structure, or do not want to choose a specific one, then we cannot employ the $T$ duality transformation: only $T^{2}$ and $S$ (and their products) are legitimate dualities.

Instead of the group $P S L_{2}(\mathbb{Z})$ of quantum dualities, we then have a duality groupoid, obtained by adding two more "nodes", which are certain topological modifications of the standard $U(1)$ twisted gauge theory $\mathcal{T}_{\kappa}^{U(1)}$. These three theories are then related to each other by various duality transformations.

More specifically, we denote the basic gauge theory $\mathcal{T}_{\kappa}^{U(1)}$ by $U(1)_{b}$. Its $T$-image will be denoted by $U(1)_{t}{ }^{20}$ In other words, we define $U(1)_{t}$ with a coupling $\kappa$ to be the same as $U(1)_{b}$ with coupling $\kappa-1$. Then $T$ maps $U(1)_{b}$ theory to $U(1)_{t}$ theory, and vice versa.

The $S$ image of $U(1)_{t}$ will be denoted as $U(1)_{s}$. It is known that $U(1)_{s}$ has an independent definition [58]: it is a gauge theory based on $\operatorname{Spin}_{\mathbb{C}}$ connections rather than standard $U(1)$ connections. Thus $S$ maps $U(1)_{t}$ to $U(1)_{s}$ theories, and vice versa. Finally, $T$ is a true duality of $U(1)_{s}$ :

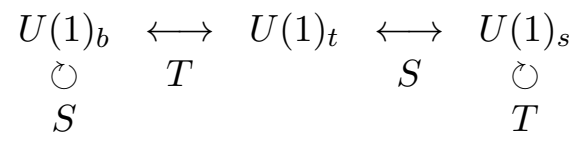

The basic boundary conditions $N_{1,0}$ and $N_{0,1}$ for the $U(1)_{b}$ theory can be defined without a choice of spin structure and are exchanged by S-duality. On the other hand, in order to define $N_{1,1}$ in $U(1)_{b}$, we cannot use $T$. Instead, we use the direct definition: $N_{1, q}$ can be defined as a modified Neumann boundary condition with $q$ extra units of boundary ChernSimons action. For odd $q$, this definition is viable but requires a choice of spin structure at the boundary.

In general, duality transformations tell us that boundary conditions $N_{p, q}$ do not require a spin structure in $U(1)_{b}$ as long as $p q$ is even.

Applying $T$, we get by definition the corresponding statement for $U(1)_{t}$. In particular, $N_{1,0}$ requires a spin structure in $U(1)_{t}$. In $U(1)_{s}$, we expect then $N_{0,1}$ to require a spin structure as well. Indeed, Dirichlet boundary conditions for a $\operatorname{Spin}_{\mathbb{C}}$ gauge field do not have a canonical choice of trivial connection, unless a spin structure is selected.

These topological aspects do affect the categories of lines available at boundary conditions. These will be ribbon categories if the boundary conditions do not require a spin structure. If they do (as $N_{1, q}$ with odd $q$ in $U(1)_{b}$ ), then they will be spin-ribbon, i.e. the twists will be defined modulo integer multiples of $\pi$ rather than $2 \pi$.

A good example of this phenomenon is the observation that although $N_{1,1}$ and $N_{1,-1}$ are related by S-duality in $U(1)_{b}$, the categories of boundary lines are equivalent only as spinribbon categories: the objects in one category have twists $2 \pi \frac{n^{2}}{2(\kappa+1)}$, while the corresponding objects in the other categories have spin $-2 \pi \frac{n^{2}}{2\left(\kappa^{-1}+1\right)}$. The two differ by possibly half-integer multiples of $2 \pi$; namely, by $2 \pi \frac{n^{2}}{2}$.

The effects percolate also to vertex algebra calculations. If both boundary conditions and the junction itself can be defined with no reference to spin structure, the vertex algebra will be a true vertex algebra, with fields of integral dimensions (but possibly a vertex

\footnotetext{
${ }^{20}$ We should really use the $\mathcal{T}_{\kappa}^{U(1)_{b}}, \mathcal{T}_{\kappa}^{U(1)_{t}}$, etc. notation, but it is a bit cumbersome.
} 
superalgebra, including odd fields). Otherwise, the vertex algebra may include fields of half-integer conformal dimensions, which requires a choice of spin structure on the Riemann surface $X$.

We have already seen this phenomenon implicitly. The standard junction in $U(1)_{b}$ from $N_{0,1}$ to $N_{1,0}$ does not require a spin structure and supports $\widehat{\mathfrak{u}}(1)_{\kappa}$. On the other hand, the same junction in $U(1)_{t}$ must be built as a composition of junctions leading to $u(1)_{1} \times \widehat{\mathfrak{u}}(1)_{\kappa-1}$, a spin-vertex algebra.

These subtleties matter when we build compactification functors to twisted $D$-modules. This is expressed in the fact that the line bundle $\mathcal{L}$ on $\operatorname{Bun}_{U(1)}$ we used above is not defined canonically unless one selects a spin structure on $X$. It can be defined canonically as a section of a $\mathbb{Z}_{2}$ gerbe $\mathcal{G}$ on $\operatorname{Bun}_{U(1)}$ which is trivial, but not canonically so.

This is a good moment to discuss some general notions related to gerbes. Let $\Gamma$ be a finite abelian group, and $\mathcal{G}$ a $\Gamma$-gerbe on a manifold $M$. In the Cech definition, this means that given an open covering of $M$, we have on each open subset $U_{i} \subset M$ of the covering a category $\mathcal{G}\left(U_{i}\right)$ that is a torsor (in the categorical sense) over the category of $\Gamma$-bundles on $U_{i}$. Choosing a trivialization of $\mathcal{G}\left(U_{i}\right)$, we obtain $\Gamma$-bundles $\mathcal{G}_{i j}$ on the overlaps $U_{i} \cap U_{j}$ and hence an element $\mathcal{G}_{i j k}$ of $\Gamma$ for each triple overlap $U_{i} \cap U_{j} \cap U_{k}$. These elements should define a Cech two-cocycle on $M$ with values in $\Gamma$.

A standard example is the following: let $\mathcal{L}$ be a line bundle on $M$ and $k$ a positive integer. Let us choose an identification $\mathbb{Z}_{k} \simeq \mu_{k}$, the group of $k$ th roots of unity. Define the $\mathbb{Z}_{k}$-gerbe $\mathcal{G}_{\mathcal{L}, k}$ on $M$ by taking as $\mathcal{G}_{\mathcal{L}, k}\left(U_{i}\right)$ the category of $k$ th roots of $\mathcal{L}$ on $U_{i}$, i.e. line bundles $\mathcal{K}$ on $U_{i}$ such that $\mathcal{K}^{k} \simeq \mathcal{L}$. (This is a torsor for the category of $\mathbb{Z}_{k}$-bundles because any two such $\mathcal{K}$ differ by a $k$ th root of the trivial line bundle; or equivalently, a $\mathbb{Z}_{k}$-bundle.) The gerbe $\mathcal{G}_{\mathcal{L}, k}$ is trivial if and only if there exists on $M$ a $k$ th root of $\mathcal{L}$ defined on the entire $M$. If $\mathcal{G}_{\mathcal{L}, k}$ is trivial, then a trivialization of $\mathcal{G}_{\mathcal{L}, k}$ is the same as a choice of such a global $k$ th root of $\mathcal{L}$.

Given a $\Gamma$-gerbe $\mathcal{G}$ on $M$ and another group $H$ (not necessarily finite) together with a homomorphism $\Gamma \rightarrow H$ whose image is in the center of $H$, we have the notion of an $H$ bundle on $M$ modified by $\mathcal{G}$. In Cech realization, an ordinary $H$-bundle assigns to each open $U_{i}$ a torsor over the group $H\left(U_{i}\right)$. Then on overlaps $U_{i} \cap U_{j}$ we obtain $H$-valued functions which has to satisfy a one-cocycle condition on triple overlaps. An $H$-bundle on $M$ modified by $\mathcal{G}$ is defined by the same data, except that the one-cocycle condition is modified on the triple overlaps by the images of $\mathcal{G}_{i j k}$ in $H$.

If $H=\mathbb{C}^{\times}$, we obtain the notion of a line bundle modified by a $\Gamma$-gerbe $\mathcal{G}$ for each homomorphism $\Gamma \rightarrow \mathbb{C}^{\times}$. We obtain the notions of $\mathcal{O}$-modules and $\mathcal{D}$-modules on $M$ modified by $\mathcal{G}$ in a similar way.

The examples we are most interested in are as follows:

- $\mathcal{L}=K_{X}$ is the canonical line bundle on a Riemann surface $X$ and $k=2$. Then the gerbe $\mathcal{G}_{K_{X}, 2}$ of square roots of $K_{X}$ is trivial, but not canonically. Its trivialization is the same as a choice of spin structure on $X$. Let $H=\mathbb{C}^{\times}$and $\mathbb{Z}_{2} \rightarrow \mathbb{C}^{\times}$be the embedding with the image $\{ \pm 1\}$. We refer the $\mathbb{C}^{\times}$-bundles on $X$ modified by $\mathcal{G}_{K_{X}, 2}$ as $\operatorname{Spin}_{\mathbb{C}}$ bundles. More generally, if $\mathbb{Z}_{2}$ maps to the center of a group $H$, we refer to the corresponding bundles on $X$ as $\operatorname{Spin}_{H}$ bundles. 
- $\mathcal{L}=K_{\mathrm{Bun}_{G}}$ is the canonical line bundle on $\operatorname{Bun}_{G}$ and $k=2$. For $G=G L_{1}$, the corresponding gerbe $\mathcal{G}_{K_{\mathrm{Bun}_{G}}, 2}$ is the gerbe discussed in this section. For a simple Lie group $G$, the gerbe $\mathcal{G}_{K_{\mathrm{Bun}_{G}}, 2}$ has been studied in [11] and, in the setting close to ours, in [34]. This gerbe is trivial, but not canonically so if $\rho$ is not an integral weight of $G$. In that case, a trivialization can be constructed from a choice of spin structure on $X$.

- $\mathcal{L}=\mathcal{L}_{G}$, the minimal line bundle on $\operatorname{Bun}_{G}$. The corresponding gerbes are needed if we want to extend the qGL duality groupoid. In $4 \mathrm{~d}$ gauge theory language, they correspond to the theories associated to discrete $\theta$ angles (see Section 9).

We can now map the three nodes of our groupoid to three variants of the usual category of $D$-modules:

- $U(1)_{b} \rightarrow D_{\kappa}\left(\operatorname{Bun}_{U(1)}\right)$, standard twisted $D$-modules.

- $U(1)_{t} \rightarrow \mathcal{G}-D_{\kappa}\left(\operatorname{Bun}_{U(1)}\right)$, twisted $D$-modules modified by the gerbe $\mathcal{G}$.

- $U(1)_{s} \rightarrow D_{\kappa}\left(\right.$ Bun $\left._{\operatorname{Spin}_{\mathbb{C}}}\right)$, twisted $D$-modules on a modified moduli space, that of $\operatorname{Spin}_{\mathbb{C}}$ bundles on the Riemann surface $X$.

The image of $T$, tensoring with $\mathcal{L}$, clearly intertwines between the two first lines. On the other hand, the line bundle $\mathcal{L}$ is defined canonically on Bun $\operatorname{Spin}_{\mathbb{C}}$.

The image of the qGL duality $S$ maps the first category $D_{\kappa}\left(\operatorname{Bun}_{U(1)}\right)$ to itself. We conjecture it exchanges the last two categories $\mathcal{G}-D_{\kappa}\left(\operatorname{Bun}_{U(1)}\right)$ and $D_{\kappa}\left(\operatorname{Bun}_{\operatorname{Spin}_{\mathbb{C}}}\right)$.

Thus we predict that, taking into account spin subtleties, the qGL dualities have the form

$$
\begin{array}{ccccc}
D_{\kappa}\left(\operatorname{Bun}_{U(1)}\right) & \underset{T}{\circlearrowright} & \mathcal{G}-D_{\kappa^{\prime}}\left(\operatorname{Bun}_{U(1)}\right) & \longleftrightarrow & D_{\kappa^{\prime \prime}}\left(\operatorname{Bun}_{\operatorname{Spin}_{\mathbb{C}}}\right) \\
S & & & T
\end{array}
$$

\section{A Richer example: $S U(2)$ gauge theory}

The vertex algebras appearing at the junctions of boundary conditions in gauge theories related to the group $S L_{2}$ (or $S U(2)$ ) provide a particularly rich class of examples. The analysis is somewhat complicated by the choices of global form of the group, i.e. $S U(2)$ or $S O(3)$, and by spin subtleties.

Usually, it is possible to relate gauge theory configurations with different global forms of the gauge group by topological manipulations which affect the junction vertex algebras in a relatively minor way, at most adding/removing some simple auxiliary vertex algebras, such as the real free fermion vertex algebra $\bigwedge_{\mathbb{R}}$ of central charge $c_{\Lambda_{\mathbb{R}}}=\frac{1}{2}$. Recall that $\bigwedge_{\mathbb{R}}{ }^{n}=\operatorname{so}(n)_{1}$, the simple quotient of $\mathfrak{s o}(n)$ Kac-Moody at level 1 . Also, $\bigwedge_{\mathbb{R}}{ }^{2}=u(1)_{1}$.

Because of that observation, we first make some preliminary statements which are essentially insensitive to the form of the gauge group, and then refine them to sharper statements involving specific gauge groups. 
8.1. Major boundary conditions and junction vertex algebras. At the first, loose level, we can refer to families of boundary conditions $N_{p, q}$ and $D_{p, q}$. We can identify several junctions and corresponding vertex algebras for the $\mathfrak{s u}(2)$ gauge algebra from string theory constructions which directly produce orthogonal or symplectic groups or by stripping off Abelian contributions from $\mathfrak{u}(2)$ statements. We refer the reader to Sections 8 and 10 of [39] and Section 4 of [16] for details. Up to dualities, we will encounter five interesting classes of junctions and vertex algebras:

- $N_{0,1} \rightarrow N_{1,0}: \operatorname{Vir}_{\kappa}$. This is the Virasoro algebra of central charge $c_{\operatorname{Vir}_{\kappa}}=13-6 \kappa-$ $6 \kappa^{-1}$. Notice that $\operatorname{Vir}_{\kappa} \simeq \operatorname{Vir}_{\kappa^{-1}}$, compatible with $R S$ invariance of the junction.

- $N_{0,1} \rightarrow N_{2,1}: \operatorname{sVir}_{2 \kappa-1}$. The super-Virasoro algebra $\operatorname{sVir}_{\kappa}$ has central charge $c_{\mathrm{SVir}_{\kappa}}=\frac{15}{2}-3 \kappa-3 \kappa^{-1}$ and satisfies $\operatorname{sVir}_{\kappa} \simeq \operatorname{sVir}_{\kappa^{-1}}$, compatible with $R S T^{2} S$ invariance of the junction.

- $D_{0,1} \rightarrow N_{1,0}: \widehat{\mathfrak{s u}}(2)_{\kappa}$. This is the Kac-Moody algebra at critically shifted level $\kappa$. It has central charge $c_{\widehat{\mathfrak{s u}}(2)_{\kappa}}=3-6 \kappa^{-1}$.

- $D_{0,1} \rightarrow N_{2,1}: \widehat{\mathfrak{o s p}}(1 \mid 2)_{2 \kappa-1}$. The super-Kac-Moody algebra $\widehat{\mathfrak{o s p}}(1 \mid 2)_{\kappa}$ has an $\widehat{\mathfrak{s u}}(2)_{\frac{1+\kappa}{2}}$ sub-algebra. It has central charge $c_{\widehat{\mathfrak{o s p}}(1 \mid 2)_{\kappa}}=1-3 \kappa^{-1}$.

- $D_{0,1} \rightarrow D_{1,0}: \mathrm{d}(2,1 \mid-\kappa)_{1}$. This is a quotient of the super-Kac-Moody algebra based on the $\mathfrak{d}(2,1 \mid-\kappa)$ exceptional superalgebra which has sub-algebra $\widehat{\mathfrak{s u}}(2)_{\kappa+1} \times$ $\widehat{\mathfrak{s u}}(2)_{\kappa^{-1}+1} \times \mathrm{su}(2)_{1}$, where $\mathrm{su}(2)_{1}$ is the simple quotient of $\widehat{\mathfrak{s u}}(2)$ Kac-Moody at level 1. The central charge of this vertex algebra is $c_{\mathrm{d}(2,1 \mid-\kappa)_{1}}=1$. The algebra is invariant under $\kappa \rightarrow \kappa^{-1}$, compatibly with $R S$ invariance of the junction.

\begin{tabular}{|c||c|}
\hline Junction & Vertex Algebra \\
\hline \hline$N_{0,1} \rightarrow N_{1,0}$ & $\operatorname{Vir}_{\kappa}$ \\
\hline$N_{0,1} \rightarrow N_{2,1}$ & $\mathrm{sVir}_{2 \kappa-1}$ \\
\hline$D_{0,1} \rightarrow N_{1,0}$ & $\widehat{\mathfrak{s u}}(2)_{\kappa}$ \\
\hline$D_{0,1} \rightarrow N_{2,1}$ & $\widehat{\mathfrak{o s p}}(1 \mid 2)_{2 \kappa-1}$ \\
\hline$D_{0,1} \rightarrow D_{1,0}$ & $\mathrm{~d}(2,1 \mid-\kappa)_{1}$ \\
\hline
\end{tabular}

TABLE 4. A brief summary of the vertex algebras which appear at some junction in $S U(2) / S O(3)$ gauge theory.

These vertex algebras are related in interesting ways by the quantum Drinfeld-Sokolov reductions, which effectively replace $D_{0,1}$ with $N_{0,1}$ in the junctions:

- The standard $\left.\operatorname{Vir}_{\kappa}=\operatorname{DS}\left[\widehat{\mathfrak{s u}}(2)_{\kappa}\right]\right]^{21}$

\footnotetext{
${ }^{21}$ Indeed $c_{\operatorname{Vir}_{\kappa}}=c_{\widehat{\mathfrak{u u}}(2)_{\kappa}}-2-6(\kappa-2)$, where -2 is the contribution from the ghost $b c$-system and $-6(\kappa-2)$ comes from the re-definition of the stress tensor.
} 
- The relation DS $\left[\widehat{\mathfrak{o s p}}(1 \mid 2)_{\kappa}\right]=\bigwedge_{\mathbb{R}} \times \operatorname{sVir}_{\kappa}$. Here we do the DS reduction of the $\widehat{\mathfrak{s u}}(2)_{\frac{1+\kappa}{2}}$ sub-algebra, without stripping off the extra free fermion originating from the odd current of charge $\frac{1}{2}$. This extra fermion is the decoupled $\bigwedge_{\mathbb{R}}$ factor indicated above 22

- The relation $\operatorname{DS}^{(1)}\left[\mathrm{d}(2,1 \mid-\kappa)_{1}\right]=\operatorname{so}(4)_{1} \times \widehat{\mathfrak{s u}}(2)_{\kappa^{-1}}$. Here we do the DS reduction on the $\widehat{\mathfrak{s u}}(2)_{\kappa+1}$ sub-algebra, without stripping off the extra four free fermions originating from the odd current of charge $\frac{1}{2}$. These extra fermions give the so $(4)_{1}$ factor indicated above. The residual $\widehat{\mathfrak{s u}}(2)_{\kappa^{-1}+1} \times \mathrm{su}(2)_{1}$ in $\left[\mathrm{d}(2,1 \mid-\kappa)_{1}\right]$ is embedded in the obvious way in $\mathrm{so}(4)_{1} \times \widehat{\mathfrak{s u}}(2)_{\kappa^{-1}}{ }^{23}$ Similarly, $\operatorname{DS}^{(2)}\left[\mathrm{d}(2,1 \mid-\kappa)_{1}\right]=$ $\operatorname{so}(4)_{1} \times \widehat{\mathfrak{s u}}(2)_{\kappa}$.

There is also a rich web of coset/extension relations which have a natural interpretation as compositions of junctions or replacements $D_{0,1} \mapsto N_{1, k}$ :

- $D_{0,1} \rightarrow N_{1,-1} \rightarrow N_{1,0}$ : The GKO-like coset $\operatorname{Vir}_{1+\kappa^{-1}}=\frac{\widehat{\mathfrak{u}}(2)_{\kappa} \times \operatorname{su}(2)_{1}}{\widehat{\mathfrak{s u}}(2)_{\kappa+1}}$.

- $D_{0,1} \rightarrow N_{1,-2} \rightarrow N_{1,0}$ : The super-GKO-like coset $\operatorname{sVir}_{1+2 \kappa^{-1}}=\frac{\widehat{\mathfrak{u}}(2)_{\kappa} \times \operatorname{so}(3)_{1}}{\widehat{\mathfrak{s u}}(2)_{\kappa+2}}$.

- $N_{0,1} \rightarrow N_{1,0} \rightarrow N_{2,1}: \operatorname{sVir}_{2 \kappa-1} \times \bigwedge_{\mathbb{R}}$ is an extension of $\operatorname{Vir}_{\kappa} \times \operatorname{Vir}_{2-\kappa^{-1}}$.

- $D_{0,1} \rightarrow N_{1,0} \rightarrow N_{2,1}$ : The alternative coset $\operatorname{Vir}_{2-\kappa^{-1}}=\frac{\widehat{\mathfrak{o s p}}(1 \mid 2)_{2 \kappa-1}}{\widehat{\mathfrak{s u}}(2)_{\kappa}}$.

- $D_{0,1} \rightarrow N_{1,-1} \rightarrow D_{1,0}$ : The d $(2,1 \mid-\kappa)_{1}$ VA is an extension of $\widehat{\mathfrak{s u}}(2)_{\kappa+1} \times \widehat{\mathfrak{s u}}(2)_{\kappa^{-1}+1} \times$ $\mathrm{su}(2)_{1}$

- $D_{0,1} \rightarrow N_{1,-2} \rightarrow D_{1,0}: \mathrm{d}(2,1 \mid-\kappa)_{1}$ is also an extension of $\widehat{\mathfrak{s u}}(2)_{\kappa+2} \times \widehat{\mathfrak{o s p}}(1 \mid 2)_{1+2 \kappa^{-1}}$.

All these extensions are compatible with each other 24

As a preparation to restoring the global form of the gauge group, it is useful to observe which collections of modules appear in the above extensions.

- $D_{0,1} \rightarrow N_{1,-1} \rightarrow N_{1,0}: \operatorname{Vir}_{1+\kappa^{-1}} \times \widehat{\mathfrak{s u}}(2)_{\kappa+1} \subset \widehat{\mathfrak{s u}}(2)_{\kappa} \times \mathrm{su}(2)_{1}$. We can expand the right hand sides into even weight Weyl modules for $\widehat{\mathfrak{s u}}(2)_{\kappa+1}$ combined with $\operatorname{Vir}_{1+\kappa^{-1}}$ degenerate modules labelled by the same weight. The extension is based on $\mathrm{KL}_{\kappa+1}(S O(3))$.

- $D_{0,1} \rightarrow N_{1,-2} \rightarrow N_{1,0}: \operatorname{sVir}_{1+2 \kappa^{-1}} \times \widehat{\mathfrak{s u}}(2)_{\kappa+2} \subset \widehat{\mathfrak{s u}}(2)_{\kappa} \times \operatorname{so}(3)_{1}$. The extension is based on $\mathrm{KL}_{\kappa+2}(S O(3))$.

\footnotetext{
${ }^{22}$ As a check, $c_{\bigwedge_{\mathbb{R}}}+c_{\mathrm{sVir}_{\kappa}}=c_{\widehat{\mathfrak{o s p}}(1 \mid 2)_{\kappa}}-2-6\left(\frac{1+\kappa}{2}-2\right)$.

${ }^{23}$ As a check, $c_{\mathrm{d}(2,1 \mid-\kappa)_{1}}-2-6(\kappa+1-2)=4 c_{\bigwedge_{\mathbb{R}}}+c_{\widehat{\mathfrak{s u}}(2)_{\kappa}-1}$.

24 There are also compatibility conditions between DS reductions and extensions. For example, the DS ${ }^{(1)}$ reduction of $\mathrm{d}(2,1 \mid-\kappa)_{1}$, i.e. $\operatorname{so}(4)_{1} \times \widehat{\mathfrak{s u}}(2)_{\kappa^{-1}}$, is an extension of $\operatorname{Vir}_{\kappa+1} \times \widehat{\mathfrak{s u}}(2)_{\kappa^{-1}+1} \times \operatorname{su}(2)_{1}$. Similarly, the $\operatorname{DS}^{(2)}$ reduction of $\mathrm{d}(2,1 \mid-\kappa)_{1}$, i.e. $\operatorname{so}(4)_{1} \times \widehat{\mathfrak{s u}}(2)_{\kappa}$, is an extension of $\widehat{\mathfrak{s u}}(2)_{\kappa+2} \times \operatorname{DS}\left[\widehat{\mathfrak{o s p}}(1 \mid 2)_{1+2 \kappa^{-1}}\right]$. Another compatibility relation is the observation that the DS reduction of $\widehat{\mathfrak{o s p}}(1 \mid 2)_{\kappa}$, i.e. $\bigwedge_{\mathbb{R}} \times \mathrm{sVir}_{\kappa}$, is an extension of $\operatorname{Vir}_{\frac{1+\kappa-1}{2}} \times \operatorname{Vir}_{\frac{1+\kappa}{2}}$.
} 
- $N_{0,1} \rightarrow N_{1,0} \rightarrow N_{2,1}: \operatorname{sVir}_{2 \kappa-1} \times \bigwedge_{\mathbb{R}}$ is an extension of $\operatorname{Vir}_{\kappa} \times \operatorname{Vir}_{2-\kappa^{-1}}$. Here the fields of half-integral/integral spin arise from products of modules labelled by odd/even weights. The extension is based on $\mathrm{KL}_{\kappa}(S U(2))$.

- $D_{0,1} \rightarrow N_{1,0} \rightarrow N_{2,1}: \operatorname{Vir}_{2-\kappa^{-1}} \times \widehat{\mathfrak{s u}}(2)_{\kappa} \subset \widehat{\mathfrak{o s p}}(1 \mid 2)_{2 \kappa-1}$. The extension is based on $K L_{\kappa}(S U(2))$.

- $D_{0,1} \rightarrow N_{1,-1} \rightarrow D_{1,0}: \widehat{\mathfrak{s u}}(2)_{\kappa+1} \times \widehat{\mathfrak{s u}}(2)_{\kappa^{-1}+1} \times \mathrm{su}(2)_{1} \subset \mathrm{d}(2,1 \mid-\kappa)_{1}$. The right hand side is a sum of products of Weyl modules for the three current algebras with the same weight, defined modulo 2 for $\mathrm{su}(2)_{1}$. We can think about the extension being based on either $\mathrm{KL}_{\kappa^{ \pm 1}+1}(S U(2))$. We also have $\widehat{\mathfrak{s u}}(2)_{\kappa+1} \times \widehat{\mathfrak{s u}}(2)_{\kappa^{-1}+1} \subset \frac{\mathrm{d}(2,1 \mid-\kappa)_{1}}{\mathrm{su}(2)_{1}}$, based on $K L_{\kappa^{ \pm 1}+1}(S O(3))$.

- $D_{0,1} \rightarrow N_{1,-2} \rightarrow D_{1,0}: \widehat{\mathfrak{s u}}(2)_{\kappa+2} \times \widehat{\mathfrak{o s p}}(1 \mid 2)_{1+2 \kappa^{-1}} \subset \mathrm{d}(2,1 \mid-\kappa)_{1}$. The extension is based on $\mathrm{KL}_{\kappa+2}(S O(3))$.

The extensions above demonstrate some important facts about the $K L_{\kappa}$ categories, seen as spin-ribbon categories ${ }^{25}$

- There is an algebra object in $K L_{\kappa+1}(S O(3)) \otimes K L_{\kappa^{-1}+1}(S O(3))$. Equivalently, $K L_{\kappa}(S O(3))$ depends on $\kappa^{-1}$ modulo 1 .

- There is an algebra object in $K L_{\kappa}(S U(2)) \otimes K L_{\frac{\kappa}{2 \kappa-1}}(S U(2))$. Equivalently, $K L_{\kappa}(S U(2))$ depends on $\kappa^{-1}$ modulo 2.

- There is an algebra object in $K L_{\kappa+1}(S U(2)) \otimes K L_{\kappa^{-1}+1}(S U(2)) \otimes \mathrm{su}(2)_{1}-\bmod$.

Equivalently, for irrational $\kappa$, let us define a new category $\widetilde{K L}_{\kappa}(S U(2))$ as a subcategory of

$$
K L_{\kappa}(S U(2)) \otimes \mathrm{su}(2)_{1}-\bmod
$$

whose simple objects are tensor products $\mathbb{V}_{n, \kappa} \otimes L_{i, 1}$ where $n \in \mathbb{Z}_{+}, i \in\{0,1\}$ and $n \equiv i \bmod 2$ (here $L_{i, 1}, i \in\{0,1\}$, are the two simple su(2) $)_{1}$-modules, $L_{0,1}$ being the vacuum module). In other words, we "dress" objects in $K L_{\kappa}(S U(2))$ by objects with the same weight $(\bmod 2)$ in $\mathrm{su}(2)_{1}-\bmod$. There is a similar definition for rational $\kappa$ as well. Then $\widetilde{K L}_{\kappa}(S U(2))$ to equivalent to $K L_{\kappa^{\prime}}(S U(2))$ with $\kappa^{-1}-$ $\left(\kappa^{\prime}\right)^{-1}=1$.

The first and second facts have a natural interpretation. The category $K L_{-\kappa^{-1}}(S O(3))$ should be equivalent to the Whittaker category for $S U(2)$, i.e. $\mathcal{C}_{\kappa}^{S U(2)}\left(N_{0,1}\right)$. The latter is naturally invariant under $T: \kappa \rightarrow \kappa+1$. On the other hand, $K L_{-\kappa^{-1}}(S U(2))$ should be equivalent to $\mathcal{C}_{\kappa}^{S O(3)}\left(N_{0,1}\right)$, which is only invariant under $T^{2}: \kappa \rightarrow \kappa+2$. Indeed, we have $n(S U(2))=1$ and $n(S O(3))=2$.

The third fact, instead, should be thought of as giving a hint about the nature of $N_{1,-1}^{S O(3)}$. Recall that this boundary condition cannot be obtained from $N_{1,0}^{S O(3)}$ by a $T$ transformation,

\footnotetext{
${ }^{25}$ The algebra objects below are really superalgebra objects, as the resulting extensions are super-vertex algebras. Regardless of whether we work with ribbon or spin-ribbon categories, all categories we use are assumed to be $\mathbb{Z}_{2}$-graded.
} 
as that is not a valid duality. Instead, it must be defined as the $S$ image of $N_{1,1}^{S U(2)}$. In particular, we have

$$
\mathcal{C}_{\kappa}^{S O(3)}\left(N_{1,-1}\right)=\widetilde{K L}_{\kappa+1}(S U(2))
$$

The following analysis requires more physics background than the rest of the paper. In the $S U(2)$ gauge theory, the boundary conditions $N_{1, q}^{S U(2)}$ can be given a direct definition as Neumann boundary conditions modified by a Chern-Simons coupling. In the $S O(3)$ theory one would expect a similar direct definition of $N_{1, q}^{S O(3)}$. However, the required Chern-Simons coupling is only well-defined for even $q$.

The construction of $\widetilde{K L}_{\kappa+1}(S U(2))$ as a sub-category in $K L_{\kappa+1}(S U(2)) \times \mathrm{su}(2)_{1}-\bmod$ suggests a direct construction of $N_{1,2 k+1}^{S O(3)}$ in four steps:

(i) Extend the gauge group from $S O(3)$ to $S U(2)$ at the boundary.

(ii) Add $2 k+1$ units of boundary Chern-Simons coupling for the bulk gauge fields.

(iii) Add an extra 3d TFT at the boundary: the TFT associated to $U(1)_{2}$ or $S U(2)_{1}$ Chern-Simons theory.

(iv) Restrict the boundary lines to the combinations of the original boundary Wilson lines and $S U(2)_{1}$ Wilson lines of the same weight $(\bmod 2)$. This can be done by "gauging a $\mathbb{Z}_{2}$ one-form symmetry", the non-anomalous combination of the boundary $\mathbb{Z}_{2}$ one-form symmetry associated to the extension of the boundary gauge group and the $\mathbb{Z}_{2}$ one-form symmetry of $S U(2)_{1}$ Chern-Simons theory.

This direct physical construction helps understand the physical origin of some of the junctions we will encounter below.

8.2. More details on modules. Recall that an $\widehat{\mathfrak{s u}}(2)_{\kappa}$ Weyl module of highest weight $m$ has a highest weight vector of conformal dimension

$$
\Delta_{m}^{\widehat{\mathfrak{s u}}(2)_{\kappa}}=\frac{m(m+2)}{4 \kappa}
$$

This makes it manifest that the topological twist $2 \pi \Delta$ is invariant for $\kappa^{-1} \rightarrow \kappa^{-1}+1$ if the weight is even, but not if the weight is odd.

The topological twist will be invariant under $\kappa^{-1} \rightarrow \kappa^{-1}+2$ for all weights, but only in the spin sense. As the dimensions of the highest weight vector of the $\mathrm{su}(2)_{1}$ module $L_{1,1}$ of highest weight 1 is $\frac{1}{4}$, we see how it can be used to compensate the effect of $\kappa^{-1} \rightarrow \kappa^{-1}+1$ on Weyl modules of odd weight.

The objects of the category $\operatorname{Vir}_{\kappa}$-mod that are images of the objects of

$$
\mathcal{C}_{\kappa}^{S U(2)}\left(N_{0,1}\right) \otimes \mathcal{C}_{\kappa}^{S U(2)}\left(N_{1,0}\right) \simeq \mathrm{KL}_{\kappa^{-1}}(S O(3)) \otimes \mathrm{KL}_{\kappa}(S U(2))
$$

under the functor $F_{\kappa}^{S U(2)}\left(N_{0,1} \rightarrow N_{1,0}\right)$ should be given by the fully degenerate modules for Virasoro algebra with conformal dimensions

$$
\Delta_{m, e}^{\mathrm{Vir}}=\left(-\frac{e}{2 b}-\frac{m b}{2}\right)\left(b+b^{-1}+\frac{e}{2 b}+\frac{m b}{2}\right)=\frac{e(e+2)}{4 \kappa}+\frac{m(m+2)}{4} \kappa-\frac{e m+e+m}{2} .
$$


with arbitrary $e$ and even $m$. The half-integral shifts compared with the dimensions of Weyl modules are OK as long as we work with spin-ribbon categories.

Notice that the full collection of modules, with arbitrary weights $e$ and $m$, does not give an image of $\mathrm{KL}_{\kappa^{-1}}(S U(2)) \otimes \mathrm{KL}_{\kappa}(S U(2))$, because of the half-integral shifts which occur when $e$ and $m$ are both odd.

Finally, because of the negative shifts in the dimensions of Virasoro modules, the combination of junctions supporting Virasoro algebra is more likely to give junction vertex algebras with negative conformal dimensions compared with the combination of junctions supporting Kac-Moody algebras.

Similar considerations as above apply to categories of modules over $\widehat{\mathfrak{o s p}}(1 \mid 2)_{2 \kappa-1}$ and $\mathrm{sVir}_{2 \kappa-1}$.

The extension construction guarantees the existence of a family of modules over $\widehat{\mathfrak{o s p}}(1 \mid 2)_{2 \kappa-1}$ which realize $\mathrm{KL}_{2-\kappa^{-1}}(S O(3))$. Concretely, we are combining $\mathrm{Vir}_{2-\kappa^{-1}}$ modules with weights $(2 e, m)$ and Weyl modules of $\widehat{\mathfrak{s u}}(2)_{\kappa}$ with weights $m$. The corresponding dimensions are

$$
\Delta_{e ; m}^{\widehat{\mathfrak{o s p}}(1 \mid 2)_{2 \kappa-1}}=\frac{e(e+1) \kappa}{(2 \kappa-1)}+\frac{(m-2 e)(m+1)}{2} .
$$

which is smallest for $m=e$, with conformal dimension of highest weight vector

$$
\Delta_{e ; m}^{\widehat{\mathrm{osp}}(1 \mid 2)_{2 \kappa-1}}=\frac{e(e+1)}{2(2 \kappa-1)} .
$$

These can be identified with Weyl modules of $\widehat{\mathfrak{o s p}}(1 \mid 2)_{2 \kappa-1}$.

If we build the extension using only modules in $\mathrm{KL}_{\kappa}(S U(2))$, the result is the even part $\left(\widehat{\mathfrak{s p p}}(1 \mid 2)_{2 \kappa-1}\right)_{e}$ of $\widehat{\mathfrak{o s p}}(1 \mid 2)_{2 \kappa-1}$ (in the sense of Lie superalgebras). Then we can realize the full $\mathrm{KL}_{2-\kappa^{-1}}(S U(2))$ as a category of modules over $\left(\widehat{\mathfrak{o s p}}(1 \mid 2)_{2 \kappa-1}\right)_{e}$.

In a similar manner, we can build modules for $\operatorname{sVir}_{2 \kappa-1}$ associated to $\mathrm{KL}_{\kappa^{-1}}(S O(3)) \times$ $\mathrm{KL}_{2-\kappa^{-1}}(S O(3))$. These are well-known degenerate modules for the super-Virasoro algebra. If we consider the even subalgebra $\left(\mathrm{sVir}_{2 \kappa-1} \times \bigwedge_{\mathbb{R}}\right)_{e}$, we can realize the larger category $\mathrm{KL}_{\kappa^{-1}}(S U(2)) \otimes \mathrm{KL}_{2-\kappa^{-1}}(S U(2))$.

One final observation concerns the embedding $\mathrm{su}(2)_{1} \otimes \mathrm{su}(2)_{1} \subset \operatorname{so}(4)_{1}=\bigwedge_{\mathbb{R}}{ }^{4}$. It demonstrates the presence of an algebra object in $\mathrm{su}(2)_{1}-\bmod \otimes \mathrm{su}(2)_{1}-\bmod$, seen as spin-ribbon categories.

There are many holomorphic vertex algebras and spin-vertex algebras which have a su $(2)_{1}$ subalgebra, and which thus lead to coset algebras $V$ such that $V$-mod is canonically conjugate to $\mathrm{su}(2)_{1}-\bmod$, as a spin-ribbon or as a ribbon category 26

8.3. Choices of global form. In this subsection, we give a more detailed analysis, in which we distinguish between $S U(2)$ and $S O(3)$ while still ignoring the subtleties related to spin structures.

8.3.1. Variants of $N_{0,1} \rightarrow N_{1,0}$. The Virasoro algebra $\operatorname{Vir}_{\kappa}$ arises naturally at the junction $N_{0,1} \rightarrow N_{1,0}$ both in $S U(2)$ and in $S O(3)$ gauge theory. Recall that $\operatorname{Vir}_{\kappa}$ is invariant under $\kappa \rightarrow \kappa^{-1}$. This transformation corresponds to the duality symmetry $R S$ which exchanges

\footnotetext{
${ }^{26} \mathrm{~A}$ simple class of examples arises from lattice vertex algebras associated to (even) unimodular lattices $L$ with a vector $v$ of length 2 .
} 
$S U(2)$ and $S O(3)$. The difference between the two choices of gauge group affects the categories of boundary line defects and hence the corresponding modules $\mathrm{Vir}_{\kappa}$.

An $S U(2)$ gauge theory will have the electric line defects on $N_{1,0}$ with all possible dominant integral weights and magnetic line defects on $N_{0,1}$ with even dominant integral weights only. The corresponding families of modules in $\mathrm{Vir}_{\kappa}$ are mutually local (indeed, the modules in one family braid trivially with the modules of the other family and the fusion product of two simple modules of this kind is again a module of this kind) and so we indeed have a tensor functor

$$
\mathrm{KL}_{\kappa}(S U(2)) \otimes \mathrm{KL}_{\kappa^{-1}}(S O(3)) \rightarrow \mathrm{Vir}_{\kappa}-\bmod
$$

The reverse set-up (with electric and magnetic line defects exchanged) is true for the $S O(3)$ gauge theory, and we have

$$
\mathrm{KL}_{\kappa}(S O(3)) \otimes \mathrm{KL}_{\kappa^{-1}}(S U(2)) \rightarrow \operatorname{Vir}_{\kappa}-\bmod
$$

(both as spin-ribbon categories). Notice that modules of odd weights in the two families braid with a -1 sign. In particular, there is no functor from $\mathrm{KL}_{\kappa}(S U(2)) \otimes \mathrm{KL}_{\kappa^{-1}}(S U(2))$ to $\operatorname{Vir}_{\kappa}-\bmod$, even as spin-ribbon categories.

The action of $R S T^{-1}$ on $N_{0,1}^{S U(2)} \rightarrow N_{1,0}^{S U(2)}$ gives $N_{1,-1}^{S O(3)} \rightarrow N_{1,0}^{S O(3)}$. If the bulk coupling for the latter is $\kappa$, then the junction vertex algebra will be $\operatorname{Vir}_{1+\kappa^{-1}}$. Correspondingly, there is a natural functor from $\mathrm{KL}_{-\kappa-1}(S O(3)) \otimes \mathrm{KL}_{\kappa}(S O(3))$ to $\operatorname{Vir}_{1+\kappa^{-1}}$-mod.

On the other hand, there is no functor from $\mathrm{KL}_{-\kappa-1}(S U(2)) \otimes \mathrm{KL}_{\kappa}(S U(2))$ to $\operatorname{Vir}_{1+\kappa^{-1}-}$ mod. This fails in two ways: the dimensions (modulo $\frac{1}{2}$ ) of families of modules of (odd, even) or (even, odd) weights differ from the expected values by $-\frac{1}{4}$ and furthermore their mutual braiding has the wrong sign.

This is not surprising as a $N_{1,-1}^{S U(2)} \rightarrow N_{1,0}^{S U(2)}$ junction cannot be obtained from the basic junction $N_{0,1}^{S U(2)} \rightarrow N_{1,0}^{S U(2)}$ by any legitimate duality transformation within the $S U(2)$ gauge theory: indeed, $S T^{ \pm 1} S$ is not a legitimate duality symmetry, only $S T^{ \pm 2} S$ and their powers are.

We can seek an appropriate junction in two ways: direct gauge theory construction or composition of junctions.

(i) The physical construction of the junction requires one to place by hand some extra degrees of freedom at the junction, in the form of an holomorphic spin vertex algebra which includes $S U(2)$ currents of level 1. ${ }^{27}$ We can take four real fermions as auxiliary degrees of freedom, i.e. so $(4)_{1}$. The junction vertex algebra is thus the coset

$$
\frac{\widehat{\mathfrak{s u}}(2)_{\kappa} \times \operatorname{so}(4)_{1}}{\widehat{\mathfrak{s u}}(2)_{\kappa+1}} \simeq \operatorname{Vir}_{1+\kappa^{-1}} \times \operatorname{su}(2)_{1} .
$$

(ii) We can map the problem by $T^{2} R S$ to the construction of a junction $N_{0,1}^{S O(3)} \rightarrow$ $N_{1,1}^{S O(3)}$ at coupling $2+\kappa^{-1}$, and use the composition $N_{0,1}^{S O(3)} \rightarrow N_{1,0}^{S O(3)} \rightarrow N_{1,1}^{S O(3)}$.

\footnotetext{
${ }^{27}$ Coupling these to the gauge fields at the junction, one absorbs the gauge anomaly associated to the change in the Chern-Simons levels across the junction.
} 
The result is an extension of $\operatorname{Vir}_{2+\kappa^{-1}} \times \operatorname{Vir}_{\frac{\kappa+1}{2 \kappa+1}}$. It can be identified with

$$
\operatorname{Vir}_{1+\kappa^{-1}} \times \widetilde{\mathrm{su}}(2)_{1} \text {. }
$$

where $\widetilde{\mathrm{su}}(2)_{1}$ is the same vertex algebra as $\mathrm{su}(2)_{1}$, with a modified choice of stress tensor.

The two answers are essentially equivalent.

Simple modules over $\operatorname{Vir}_{1+\kappa^{-1}} \times \operatorname{su}(2)_{1}$ corresponding to a pair of Weyl modules $\mathbb{V}_{m, \kappa}$ and $\mathbb{V}_{n,-\kappa-1}$ from the categories of boundary line defects $\mathcal{C}_{\kappa}^{S U(2)}\left(N_{1,-1}^{S U(2)}\right)^{\vee}=\mathrm{KL}_{-\kappa-1}(S U(2))$ and $\mathcal{C}_{\kappa}^{S U(2)}\left(N_{1,0}^{S U(2)}\right)=\mathrm{KL}_{\kappa}(S U(2))$ are produced in the coset description on the right hand side of (8.6) as the BRST cohomology of the tensor product $\mathbb{V}_{m, \kappa} \otimes V \otimes \mathbb{V}_{n,-\kappa-1}$ under the diagonal action of $\widehat{\mathfrak{s u}}(2)$ of twice the critical level.

If their weights, $m$ and $n$, have the same parity, then the coset module coincides with the appropriate $\operatorname{Vir}_{\kappa}$-module dressed by the vacuum module of $\operatorname{su}(2)_{1}$ (or $V$ ). But if the weights have opposite parity, then the coset module is a $\operatorname{Vir}_{\kappa}$-module dressed by the nontrivial module of $\operatorname{su}(2)_{1}$ (or $\left.V\right)$. This gives us the desired functor

$$
\mathrm{KL}_{-\kappa-1}(S U(2)) \otimes \mathrm{KL}_{\kappa}(S U(2)) \rightarrow\left(\operatorname{Vir}_{1+\kappa^{-1}} \times V\right)-\bmod .
$$

Note that the extra dressing cancels the troublesome $-\frac{1}{4}$ shifts in conformal dimensions that would result in the undesired - sign in the mutual braiding.

A small aside: the junctions above are compatible with the tentative direct gauge theory description of $N_{1, \pm 1}^{S O(3)}$ involving an auxiliary $3 \mathrm{~d}$ TFT at the boundary. As we construct a junction involving $N_{1, \pm 1}^{S O(3)}$, we need in particular to provide a boundary condition for the 3d TFT.

Such a boundary condition naturally supports a $\mathrm{su}(2)_{1}$ vertex algebra.

(i) In a direct gauge theory construction of $N_{1,-1}^{S O(3)} \rightarrow N_{1,0}^{S O(3)}$, we can use such su(2) as auxiliary degrees of freedom at the junction, rather than an holomorphic vertex algebra such as so(4) $)_{1}$. That gives precisely the GKO construction

$$
\frac{\widehat{\mathfrak{s u}}(2)_{\kappa} \times \operatorname{su}(2)_{1}}{\widehat{\mathfrak{s u}}(2)_{\kappa+1}} \simeq \operatorname{Vir}_{1+\kappa^{-1}} .
$$

(ii) In a direct gauge theory construction of $N_{0,1}^{S O(3)} \rightarrow N_{1,-1}^{S O(3)}$, the su(2) 1 vertex algebra goes along for the ride, giving the expected $\operatorname{Vir}_{1+\kappa} \times \operatorname{su}(2)_{1}$.

\begin{tabular}{|c||c||c|}
\hline Junction & $S U(2)$ & $S O(3)$ \\
\hline \hline$N_{0,1} \rightarrow N_{1,0}$ & $\operatorname{Vir}_{\kappa}$ & $\operatorname{Vir}_{\kappa}$ \\
\hline$N_{0,1} \rightarrow N_{1,-1}$ & $\operatorname{Vir}_{1+\kappa}$ & $\operatorname{Vir}_{1+\kappa} \times \mathrm{su}(2)_{1}$ \\
\hline$N_{1,-1} \rightarrow N_{1,0}$ & $\operatorname{Vir}_{1+\kappa^{-1}} \times \mathrm{su}(2)_{1}$ & $\operatorname{Vir}_{1+\kappa^{-1}}$ \\
\hline
\end{tabular}

TABLE 5. Global form of the gauge group and junctions, part 1. 
8.3.2. Variants of $N_{0,1} \rightarrow N_{2,1}$. The simplest junction of this type occurs for $N_{1,-2}^{S O(3)} \rightarrow$ $N_{1,0}^{S O(3)}$ and its duality images, such as $N_{0,1}^{S U(2)} \rightarrow N_{2, \pm 1}^{S U(2)}$, etc. In the former conventions, it supports a $\mathrm{SVir}_{1+2 \kappa^{-1}}$ vertex algebra, though in many constructions it emerges dressed by an extra decoupled free fermion. This is the case, for example, of the composition $N_{0,1}^{S U(2)} \rightarrow N_{1,0}^{S U(2)} \rightarrow N_{2,1}^{S U(2)}$.

A direct gauge theory construction of a $N_{1,-2}^{S O(3)} \rightarrow N_{1,0}^{S O(3)}$ junction requires a choice of auxiliary holomorphic spin-vertex algebra with a su(2) $)_{2}$ sub-algebra 28 The simplest choice is $\mathrm{so}(3)_{1}$, which leads to a junction vertex algebra given by the super-GKO-like coset

$$
\operatorname{sVir}_{1+2 \kappa^{-1}}=\frac{\widehat{\mathfrak{s u}}(2)_{\kappa} \times \operatorname{so}(3)_{1}}{\widehat{\mathfrak{s u}}(2)_{\kappa+2}}
$$

The lines of even weight on both boundaries end on appropriate coset modules, which are the degenerate modules of sVir.

Another interesting junction is $N_{0,1}^{S O(3)} \rightarrow N_{2,1}^{S O(3)}$. Junction composition $N_{0,1}^{S O(3)} \rightarrow$ $N_{1,0}^{S O(3)} \rightarrow N_{2,1}^{S O(3)}$ now produces the even subalgebra $\left(\operatorname{sVir}_{2 \kappa-1} \times \bigwedge_{\mathbb{R}}\right)_{e}$.

Notice that the duality orbit of this junction does not include $N_{0,1}^{S O(3)} \rightarrow N_{2,-1}^{S O(3)}$, which will require an alternative construction. Instead, it includes $N_{1,2}^{S U(2)} \rightarrow N_{1,0}^{S U(2)}, N_{1,1}^{S U(2)} \rightarrow$ $N_{1,-1}^{S U(2)}$ and $N_{1,-1}^{S O(3)} \rightarrow N_{1,1}^{S O(3)}$.

The latter provides another perspective on the junction: the boundary conditions for the $3 \mathrm{~d}$ TFTs involved in $N_{1,-1}^{S O(3)}$ and $N_{1,1}^{S O(3)}$ can provide $\mathrm{su}(2)_{1} \times \mathrm{su}(2)_{1}$ auxiliary degrees of freedom, resulting in the coset description

$$
\left(\operatorname{sVir}_{1+2 \kappa^{-1}} \times \bigwedge_{\mathbb{R}}\right)_{e}=\frac{\widehat{\mathfrak{s u}}(2)_{\kappa} \times \mathrm{su}(2)_{1} \times \mathrm{su}(2)_{1}}{\widehat{\mathfrak{s u}}(2)_{\kappa+2}}
$$

In order to study the final duality orbit, including $N_{0,1}^{S O(3)} \rightarrow N_{2,-1}^{S O(3)}$, we can look at $N_{1,-2}^{S U(2)} \rightarrow N_{1,0}^{S U(2)}$. A direct gauge theory construction requires an auxiliary holomorphic vertex algebra which has a $\mathrm{su}(2)_{2}$ sub-algebra, but also includes fields of odd weight, so that all boundary lines can end at the junction and we can find a fully faithful functor from the boundary categories into the junction vertex algebra modules.

There are many choice of such a vertex algebra, and none is obviously canonical. We can realize $\mathrm{su}(2)_{2}$ as the diagonal combination of two $\mathrm{su}(2)_{1}$ 's, each included in a separate so $(4)_{1}$ factor.

We can describe the resulting junction vertex algebra as a coset

$$
\frac{\widehat{\mathfrak{s u}}(2)_{\kappa} \times \operatorname{so}(8)_{1}}{\widehat{\mathfrak{s u}}(2)_{\kappa+2}}
$$

where $\widehat{\mathfrak{s u}}(2)$ is embedded in the $\operatorname{so}(8)_{1}$ in a non-trivial way, acting separately on two blocks of four fermions each. With a bit of care, we can simplify that to

$$
\left(\operatorname{sVir}_{1+2 \kappa^{-1}} \times \operatorname{so}(5)\right)_{e}
$$

\footnotetext{
${ }^{28}$ This is required in order to compensate for the shift of boundary Chern-Simons coupling (note that the level of the $\widehat{\mathfrak{s u}}(2)$ Kac-Moody algebra in $\operatorname{so}(3)_{1}$ is equal to $\left.2=n(S O(3))\right)$.
} 
We can obtain the same result by a composition $N_{1,-2}^{S U(2)} \rightarrow N_{1,-1}^{S U(2)} \rightarrow N_{1,0}^{S U(2)}$ leading to an extension of $\operatorname{Vir} \times \mathrm{su}(2)_{1} \times \operatorname{Vir} \times \mathrm{su}(2)_{1}$.

\begin{tabular}{|c||c||c|}
\hline Junction & $S U(2)$ & $S O(3)$ \\
\hline \hline$N_{1,-2} \rightarrow N_{1,0}$ & $\left(\mathrm{sVir}_{1+2 \kappa^{-1}} \times \mathrm{SO}(5)_{1}\right)_{e}$ & $\operatorname{sVir}_{1+2 \kappa^{-1}}$ \\
\hline$N_{1,2} \rightarrow N_{1,0}$ & $\left(\mathrm{sVir}_{-1+2 \kappa^{-1}} \times \bigwedge_{\mathbb{R}}\right)_{e}$ & $\mathrm{sVir}_{-1+2 \kappa^{-1}}$ \\
\hline$N_{1,-1} \rightarrow N_{1,1}$ & $\left(\operatorname{sVir}_{\frac{\kappa-1}{\kappa+1}} \times \mathrm{so}(5)_{1}\right)_{e}$ & $\left(\mathrm{sVir}_{\frac{\kappa-1}{\kappa+1}} \times \bigwedge_{\mathbb{R}}\right)_{e}$ \\
\hline
\end{tabular}

TABLE 6. Global form of the gauge group and junctions, part 2.

8.3.3. Variants of $D_{0,1} \rightarrow N_{1,0}$. We know that both $D_{0,1}^{S U(2)} \rightarrow N_{1,0}^{S U(2)}$ and $D_{0,1}^{S O(3)} \rightarrow$ $N_{1,0}^{S O(3)}$ support the Kac-Moody algebra $\widehat{\mathfrak{s u}}(2)_{\kappa}$. Upon compactification, these junctions produce the expected $\mathcal{D}_{\kappa}$ in $D_{\kappa}\left(\operatorname{Bun}_{S L_{2}}\right)$ or in $D_{\kappa}\left(\operatorname{Bun}_{P S L_{2}}\right)$.

Very similar considerations apply to $D_{0,1}^{S U(2)} \rightarrow N_{1, k}^{S U(2)}$. These junctions support $\widehat{\mathfrak{s u}}(2)_{\kappa-k}$. Upon compactification, these junctions produce $\mathcal{D}_{\kappa-k} \otimes \mathcal{L}^{k}$ in $D_{\kappa}\left(\operatorname{Bun}_{S L_{2}}\right)$.

The same is true for $D_{0,1}^{S O(3)} \rightarrow N_{1,2 k}^{S O(3)}$. These junctions support $\widehat{\mathfrak{s u}}(2)_{\kappa-2 k}$. Upon compactification, these junctions produce $\mathcal{D}_{\kappa-2 k} \otimes \mathcal{L}^{2 k}$ in $D_{\kappa}\left(\operatorname{Bun}_{P S L_{2}}\right)$.

A separate class of interesting junctions is $D_{0,1}^{S O(3)} \rightarrow N_{1,2 k-1}^{S O(3)}$, which cannot be produced by the action of $T^{2}$ on the standard junction. Instead, we can consider the extension $D_{0,1}^{S O(3)} \rightarrow N_{1,0}^{S O(3)} \rightarrow N_{1,1}^{S O(3)}$, leading to $\widehat{\mathfrak{s u}}(2)_{\kappa-1} \otimes \mathrm{su}(2)_{1}$, coupled to $P S L_{2}$ bundles by the diagonal combination of the currents.

Upon compactification, this junction produces an interesting object in $D_{\kappa}\left(\operatorname{Bun}_{P S L_{2}}\right)$, which should be qGL dual to $\mathcal{D}_{-\kappa^{-1}+1} \otimes \mathcal{L}^{-1}$ in $D_{-\kappa^{-1}}\left(\operatorname{Bun}_{S L_{2}}\right)$.

8.3.4. Variants of $D_{0,1} \rightarrow N_{2,1}$. First, we can look at $D_{0,1}^{S U(2)} \rightarrow N_{2, \pm 1}^{S U(2)}$. This should support $\widehat{\mathfrak{o s p}}(1 \mid 2)_{2 \kappa \mp 1}$. Upon compactification, this junction should produce a rather nontrivial object: the restriction of $\mathcal{D}_{2 \kappa-1}\left[\operatorname{Bun}_{O S p_{1 \mid 2}}\right]$ to $\operatorname{Bun}_{S L_{2}}$.

We thus expect this object to be qGL dual to $\mathcal{D}_{-\kappa^{-1} \pm 2} \otimes \mathcal{L}^{\mp 2}$ in $D_{-\kappa^{-1}}\left(\operatorname{Bun}_{P S L_{2}}\right)$.

For $S O(3)$ gauge group, we should distinguish two junctions not related by dualities:

(i) $D_{0,1}^{S O(3)} \rightarrow N_{2,1}^{S O(3)}$ : the composition of junctions involving $N_{1,0}^{S O(3)}$ only gives the (Grassmann) even part $\left(\widehat{\mathfrak{o s p}}(1 \mid 2)_{2 \kappa-1}\right)_{e}$.

(ii) $D_{0,1}^{S O(3)} \rightarrow N_{2,-1}^{S O(3)}$ : the composition of junctions involving $N_{1,-1}^{S O(3)}$ gives instead $\left(\widehat{\mathfrak{o s p}}(1 \mid 2)_{2 \kappa+1} \times \operatorname{so}(4)_{1}\right)_{e}$.

8.3.5. Kernel junctions. Now we have the information needed to study a junction $D_{1.0}^{S U(2)} \rightarrow$ $D_{1,0}^{S U(2)}$ or, equivalently, $D_{0,1}^{S O(3)} \rightarrow D_{1,0}^{S O(3)}$. We can use the composition $D_{0,1}^{S U(2)} \rightarrow N_{1,-1}^{S U(2)} \rightarrow$ $D_{1,0}^{S U(2)}$. Now the second junction has an extra $\mathrm{su}(2)_{1}$ factor and the composition gives precisely $\mathrm{d}(2,1 \mid-\kappa)_{1}$. 
Crucially, the coupling to $S L_{2}$ bundles associated to $D_{0,1}^{S U(2)}$ employs the $\widehat{\mathfrak{s u}}(2)_{\kappa+1}$ subalgebra, but the coupling to $P S L_{2}$ bundles associated to $D_{1,0}^{S U(2)}$ employs the diagonal sub-algebra of the $\widehat{\mathfrak{s u}}(2)_{\kappa^{-1}+1} \times \operatorname{su}(2)_{1}$ sub-algebra.

This observation is very important: the currents in $\mathrm{d}(2,1 \mid-\kappa)_{1}$ have either (even, even, even) or (odd, odd,odd) weights for the three subalgebras. Under the diagonal sub-algebra of the $\widehat{\mathfrak{s u}}(2)_{\kappa^{-1}+1} \times \operatorname{su}(2)_{1}$, though, they have even weights and thus the coupling to $P S L_{2}$ bundles is possible.

The sheaf of coinvariants of $\mathrm{d}(2,1 \mid-\kappa)_{1}$ is an object in $D_{\kappa+1}\left(\operatorname{Bun}_{S L_{2}}\right) \otimes D_{\kappa^{-1}+2}\left(\operatorname{Bun}_{P S L_{2}}\right)$ which can be mapped canonically (up to spin subtleties) to an object in $D_{\kappa}\left(\operatorname{Bun}_{S L_{2}}\right) \otimes$ $D_{\kappa^{-1}}\left(\operatorname{Bun}_{P S L_{2}}\right)$, which is the conjectural qGL kernel.

An interesting variant is the junction $D_{0,1}^{S O(3)} \rightarrow D_{1,1}^{S O(3)}$. We can use the composition $D_{0,1}^{S O(3)} \rightarrow N_{1,0}^{S O(3)} \rightarrow D_{1,1}^{S O(3)}$. The second junction can be dualized to $N_{0,1}^{S U(2)} \rightarrow D_{1,-1}^{S U(2)}$ and then to $N_{0,1}^{S U(2)} \rightarrow D_{1,0}^{S U(2)}$ and thus $D_{0,1}^{S O(3)} \rightarrow N_{1,0}^{S O(3)}$. The composition gives a smaller vertex algebra: the coset $\frac{\mathrm{d}(2,1 \mid-\kappa)_{1}}{\mathrm{su}(2)_{1}}$.

8.4. Spin subtleties. Finally, we include spin structures in our analysis. In the absence of a spin structure, the $S U(2)$ gauge theory is still $T$ invariant. It comes in two variants, though, as we can define a standard theory $S U(2)_{b}$ based on an $S U(2)$ connection and a twisted theory $S U(2)_{s}$ based on an $\operatorname{Spin}_{\mathbb{C}}$-like $S U(2)$ connections.

On the other hand, $S O(3)$ gauge theory is invariant under $T^{4}$ rather than $T^{2}$. We can denote the corresponding two versions of $S O(3)$ gauge theory as $S O(3)_{b}$ and $S O(3)_{t}$, related by $T^{2}$.

Perhaps surprisingly, it is natural to take $S O(3)_{t}$ and $S O(3)_{b}$ to be respectively the $S$-dual images of $S U(2)_{b}$ and $S U(2)_{s}$. We thus have the following duality groupoid:

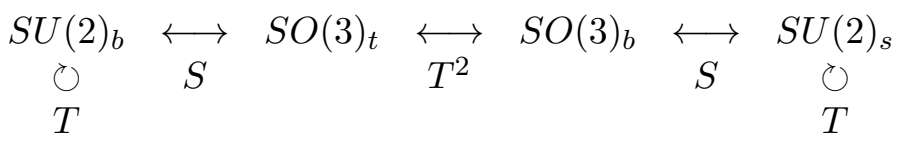

In this convention, Neumann boundary condition $N_{1,0}$ does not require a spin structure for $S O(3)_{b}$ but does require it for $S O(3)_{t}$. Indeed, the Nahm pole boundary condition for $S U(2)_{b}$ requires a choice of spin structure, but does not for $S U(2)_{s}$.

On the other hand, Neumann boundary condition $N_{1,0}$ does not require a choice of spin structure for either $S U(2)$ theory, nor does Nahm for either $S O(3)$ theory.

Acting with $T$, we find that $N_{1, \pm 1}$ do not require a choice of spin structure for either $S U(2)$ theory and thus should not require a spin structure for either $S O(3)$ theory as well.

On the other hand, acting with $T^{2}$ we find that $N_{1, \pm 2}$ require a spin structure for $S O(3)_{b}$ but not for $S O(3)_{t}$. Acting with $S$, we find that $N_{2, \pm 1}$ require a spin structure for $S U(2)_{s}$ but not for $S U(2)_{b}$. This makes sense: the boundary hypermultiplets are twisted into fields of integral spin, but transform as a doublet of $S U(2)$. They are not naturally sections of Spin $_{\mathbb{C}^{-}}$like $S U(2)$ bundle. On the other hand, the $N_{2, \pm 1}$ should not require a spin structure for either $S O(3)$ theory. 
Standard Dirichlet boundary conditions only require a spin structure for $S U(2)_{s}$, and even then it is better to think about coupling to a background $\operatorname{Spin}_{\mathbb{C}}$-like $S U(2)$ bundle at the boundary.

Upon compactification on a Riemann surface, we can map the four nodes of our groupoid to four variants of the usual category of $D$-modules:

- $S U(2)_{b} \rightarrow D_{\kappa}\left(\operatorname{Bun}_{S L_{2}}\right)$, standard twisted $D$-modules.

- $S O(3)_{b} \rightarrow D_{\kappa}\left(\operatorname{Bun}_{P S L_{2}}\right)$, standard twisted $D$-modules.

- $S O(3)_{t} \rightarrow \mathcal{G}-D_{\kappa}\left(\operatorname{Bun}_{P S L_{2}}\right)$, twisted $D$-modules modified by the gerbe $\mathcal{G}$.

- $S U(2)_{s} \rightarrow D_{\kappa}\left(\right.$ Bun $\left._{\text {Spin-SL }}\right)$, twisted $D$-modules on a modified moduli stack, that of $\operatorname{Spin}_{S L_{2}}$ bundles on the Riemann surface $X$ (we use notation from Section 7.4).

This agrees with the duality statements in 34 .

As an example of non-spin junctions, the $N_{0,1}^{S O(3)_{b}} \rightarrow N_{1,0}^{S O(3)_{b}}$ or $N_{0,1}^{S U(2)_{s}} \rightarrow N_{1,0}^{S U(2)_{s}}$ junctions can be defined with no reference to spin structure and indeed support the standard $\operatorname{Vir}_{\kappa}$ algebra.

It is straightforward, if tedious, to build more examples of junctions which do not require a spin structure.

Instead, we will just make a couple of observations about the duality kernel vertex algebra.

- The conformal blocks of $\mathrm{d}(2,1 \mid-\kappa)_{1}$ will give an object in $D_{\kappa+1}\left(\operatorname{Bun}_{S L_{2}}\right) \otimes$ $D_{\kappa^{-1}+2}\left(\operatorname{Bun}_{P S L_{2}}\right)$. Because $\mathcal{L}^{2}$ is a section of the gerbe $\mathcal{G}$, this maps to an object in $D_{\kappa}\left(\operatorname{Bun}_{S L_{2}}\right) \otimes \mathcal{G}-D_{\kappa^{-1}}\left(\operatorname{Bun}_{P S L_{2}}\right)$, as needed.

- Consider the subalgebra $\left(\mathrm{d}(2,1 \mid-\kappa)_{1} \times \mathrm{so}(3)_{1}\right)_{e}$. This vertex algebra can be coupled to $\operatorname{Spin}_{S L_{2}}$ bundles. If we couple the second factor to the $P S L_{2}$ bundles, the conformal blocks will give an object in $D_{\kappa+1}\left(\operatorname{Bun}_{s-S L_{2}}\right) \otimes D_{\kappa^{-1}+4}\left(\operatorname{Bun}_{P S L_{2}}\right)$. This maps canonically to an object in $D_{\kappa}\left(\operatorname{Bun}_{s-S L_{2}}\right) \otimes D_{\kappa^{-1}}\left(\operatorname{Bun}_{P S L_{2}}\right)$, as needed.

\section{General gauge groups and Discrete $\theta$ angles}

Until now, we have employed a somewhat traditional perspective on the S-duality groupoid, in which we focus on the standard $4 \mathrm{~d}$ supersymmetric gauge theories for a simple compact Lie group $G_{c}$ and its Langlands dual ${ }^{L} G_{c}$.

The lift of the duality group from spin-TFTs to TFTs for the groups $U(1)$ and $S U(2) / S O(3)$ forced us to consider some generalizations of that structure, either involving $\operatorname{Spin}_{\mathbb{C}}$-like modifications of the gauge group or the images of the standard gauge theories under the duality transformation $T$ (we will call them $T$-images).

Even if we remain in the realm of spin-TFTs, it turns out that extending the duality groupoid by introducing $T$-images of standard gauge theories, however useful, is still incomplete [38, 8]. It turns out that the definition of $4 \mathrm{~d}$ gauge theories can be modified by a variety of "discrete $\theta$ angles" and some of the resulting modifications are not simply the $T^{k}$-images of standard gauge theories. Furthermore, there is a full duality groupoid generated by $T$ and $S_{m}$, whose nodes can be identified with a variety of thus modified gauge theories. 
The full duality groupoid organizes all theories associated to the Lie algebras $\mathfrak{g}$ and ${ }^{L_{\mathfrak{g}}}$ into a complicated pattern of orbits, whose structure depends sensitively on the specific gauge Lie algebra.

When such modified $4 \mathrm{~d}$ gauge theories are compactified on a Riemann surface $\Sigma$, we obtain as the result certain modifications of the sigma model with the target $\mathcal{M}_{H}(G)$, the Hitchin moduli space associated to $\Sigma$ and $G$. As a result, the corresponding categories of twisted $D$-modules on $\mathrm{Bun}_{G}$ get modified in such a way that each connected component of $\operatorname{Bun}_{G}$ gets equipped with an appropriate discrete $B$-field and the corresponding categories of $D_{\kappa}$-modules get twisted by appropriate gerbes. As a result, we obtain a variety of duality relations involving these gerbe-twisted categories of $D_{\kappa}$-modules.

This overall structure is reasonably well understood for the physical gauge theories, but has to be further refined for the topologically twisted gauge theories, because of spin subtleties.

There is some recent mathematical work [49, 48] which describes modifications of the standard Geometric Langlands correspondence that appear to be closely related to the modifications due to the discrete $\theta$ angles. It would be very interesting to make the dictionary explicit and compare the expected actions of S-duality on these data.

9.1. Topological actions and lattice of line defects. In order to consider topological modifications of gauge theory actions (a.k.a. "discrete $\theta$-angles") in full generality, one has to study certain generalized cohomology theories of $B G$.

In the standard physical gauge theory, these generalized cohomology theories will be associated to cobordisms of spin manifolds equipped with a $G$ bundle and perhaps an $S U(4)_{R}$-bundle.

Alternatively, as the spinors in the physical theory transform in the fundamental representation of the $S U(4)_{R} \simeq \operatorname{Spin}(6)_{R}$ R-symmetry group, while fields of integral spin transform in the representations of $S O(6)_{R}$, it should be possible to couple the theory to $\operatorname{Spin}_{S U(4)_{R}{ }^{-}}$ bundles, i.e. bundles on a four-manifold $M$ with the structure group $(\operatorname{Spin}(4) \times S U(4)) / \mathbb{Z}_{2}$ (which is a $\mathbb{Z}_{2}$-extension of the structure group $S O(4) \times\left(S U(4) / \mathbb{Z}_{2}\right)=S O(4) \times S O(6)$ ), such that the corresponding $S O(4) \times\left(S U(4) / \mathbb{Z}_{2}\right)$-bundle has the frame bundle of $M$ along the first factor. These should be available on all manifolds. Topological actions for this variant of the physical theories will be classified by some cohomology theories associated to cobordisms of manifolds equipped with a $G$-bundle and an $\operatorname{Spin}_{S U(4)_{R}}$-bundle.

The latter choice is probably better in preparation for a topological twist. It suggests that the spin-refined duality action, which we will explore later on, may already be probed within the physical theory.

Upon topological twist, the classification of discrete $\theta$-angles should simplify a bit, as the cohomology theories will be associated to cobordisms of manifolds equipped with a $G$-bundle only and should coincide with some standard version of the group cohomology $H^{4}(B G, U(1))$.

The group cohomology description of the topological action is well-suited for understanding the compactification to the $2 \mathrm{~d}$ sigma model on a Riemann surface $\Sigma$ with the target Hitchin moduli space $\mathcal{M}_{H}(G)$. Indeed, there is an obvious map $\Sigma \times \operatorname{Bun}_{G}(\Sigma) \rightarrow B G$ and the pull-back of the class in $H^{4}(B G, U(1))$ along that map can be integrated over $\Sigma$ to give 
a class in $H^{2}\left(\operatorname{Bun}_{G}(\Sigma), U(1)\right)$, i.e. a discrete $B$-field, or equivalently a gerbe, of the sigma model 29

Let us denote a choice of the theory as a pair $(G, \omega)$ with $\omega$ being a choice of topological action added on top of the usual gauge theory action. An important open question is to describe explicitly the action of the duality groupoid on pairs $(G, \omega)$. This has not yet been done, as far as we know 30 What is known is the action of the duality groupoid there on a simpler piece of data obtained from pairs $(G, \omega)$. It seems to capture the information hidden in the pair $(G, \omega)$ that we need, up to the spin subtleties.

This piece of data is the lattice $\Lambda$ of allowed electric and magnetic charges for the line defects in the theory. We will use this data even though these line defects are actually not available in general in the bulk of the topologically twisted theory (only at the boundary), as a formal proxy for the underlying topological action $\omega$ of the gauge theory. If we further add to $\Lambda$ the data of the topological spin associated to the line defects, we should be able to keep track of spin subtleties.

Recall that the charges of line defects in a gauge theory with the Lie algebra $\mathfrak{g}$ are pairs $(\mathbf{m}, \mathbf{e})$ where $\mathbf{m}$ is in the magnetic weight lattice ${ }^{L} P$ (that is, the coweight lattice of $\mathfrak{g}$ ) and e is in the weight lattice $P$ of $\mathfrak{g}$.

There is a natural (Dirac) inner product on ${ }^{L} P \times P$ :

$$
\left\langle(\mathbf{m}, \mathbf{e}),\left(\mathbf{m}^{\prime}, \mathbf{e}^{\prime}\right)\right\rangle=\mathbf{m} \cdot \mathbf{e}^{\prime}-\mathbf{m}^{\prime} \cdot \mathbf{e} .
$$

By definition, a lattice $\Lambda$ of allowed line defects is a maximal local sublattice of ${ }^{L} P \times P$. Here "local" means that the restriction of the above inner product to $\Lambda$ should take integer values, and "maximal" means that it is not be possible to add any other elements to $\Lambda$ without violating locality property. In particular, it follows that $\Lambda$ necessarily contains ${ }^{L} Q \times Q$, where $Q \subset P$ and ${ }^{L} Q \subset{ }^{L} P$ are the lattices of roots and coroots, respectively. Hence such $\lambda$ is completely determined by its projection onto $\left({ }^{L} P /{ }^{L} Q\right) \times P / Q$.

The duality groupoid acts on $\Lambda$ by transforming the elements in the obvious way:

$$
S_{m}:(\mathbf{m}, \mathbf{e}) \rightarrow(\mathbf{e},-\mathbf{m}) \quad T:(\mathbf{m}, \mathbf{e}) \rightarrow(\mathbf{m}, \mathbf{e}+\mathbf{m}) .
$$

In order to make contact with the cohomology class $\omega$ discussed above, we rearrange the data in $\Lambda$ a bit. First of all, we can read off the global form of the gauge group $G$ by looking at the sublattice of Wilson lines $(\mathbf{0}, \mathbf{e})$ inside $\Lambda$, i.e. the intersection $\Lambda \cap P$. This should be the weight lattice of the gauge group $G$, which we denote by $\Lambda_{G}$. Likewise, the intersection $\Lambda \cap{ }^{L} P$ should be the coweight lattice of $G$, which we denote by $\Lambda_{G}^{M}$. For each $\mathbf{m}$ the values of allowed electric charges $\mathbf{e}$ in our theory (i.e. such that $(\mathbf{m}, \mathbf{e}) \in \Lambda$ ) then form a torsor for $\Lambda_{G}$. Picking a specific representative e of this coset, we define a pairing $\Lambda_{G}^{M} \times \Lambda_{G}^{M} \rightarrow U(1)$ by the formula

$$
\left\langle\mathbf{m}, \mathbf{m}^{\prime}\right\rangle=e^{2 \pi i \mathbf{m}^{\prime} \cdot \mathbf{e}}
$$

\footnotetext{
${ }^{29}$ Notice that this statement holds true for whatever generalized cohomology theory is relevant to the problem at hand: a topological action for the gauge theory will descend to a topological action for the $2 \mathrm{~d}$ sigma model.

${ }^{30}$ While some of the spin subtleties are expressed in $\omega$, to get a complete description of the possible theories one needs to allow $\operatorname{Spin}_{S U(4)_{R}}$-bundles in addition to $G$-bundles, as we explained above in the case of $G=U(1)$ and $S U(2) / S O(3)$.
} 
which is symmetric because of the locality property. If we identify $\Lambda_{G}^{M}$ with the possible magnetic fluxes on a sphere, this pairing is nothing but the evaluation of the corresponding cohomology class $\omega$ on the four-manifold which is the product of two spheres.

In the next subsection, we will consider examples of lattices $\Lambda$ in the case of $S L_{n}$. We will consider the projections of the lattices onto $\left({ }^{L} P /{ }^{L} Q\right) \times P / Q$.

9.2. Example: $S L_{2}$, revisited. In this case, $\left({ }^{L} P /{ }^{L} Q\right) \times(P / Q)=\mathbb{Z}_{2} \times \mathbb{Z}_{2}$ and there are three possible lattices of charges whose projections onto $\left(\mathbb{Z}_{2}, \mathbb{Z}_{2}\right)$ contain $(0,0)$ and either of the three $(0,1),(1,0)$ or $(1,1)$.

The first choice is $S U(2)$ gauge theory, the second is the standard $S O(3)$ gauge theory, which we denote by $S O(3)_{+}$and the third is the $T$-image of $S O(3)_{+}$, which we denote by $S O(3)_{-}$. The duality transformation $S$ exchanges $S U(2)$ and $S O(3)_{+}$and maps $S O(3)_{-}$to itself.

The spin-enrichment of the duality groupoid keeps track of four distinct variants of $S O(3)$, corresponding to the basic $S O(3)_{0}$ theory and to the $T^{k}$ images $S O(3)_{k}$. Now $S$ relates $S O(3)_{0}$ and Spin- $S U(2), S O(3)_{1}$ and $S O(3)_{3}, S O(3)_{2}$ and $S U(2)$.

Upon compactification on a Riemann surface, $S O(3)_{k}$ gives $D_{k}$-modules twisted by the gerbe associated to the $k$ th power of the naive line bundle $\mathcal{L}$.

9.3. Example: $S L_{N}$. Ignoring spin subtleties, the number of distinct variants of $\mathfrak{s l}_{N}$ gauge theories is equal to the sum of the divisors of $N$.

As the basic $S U(N)$ gauge theory is invariant under $\Gamma_{0}(N)$, the duality groupoid images of $S U(N)$ are counted by the index of $\Gamma_{0}(N)$ in $P S L_{2}(\mathbb{Z})$, which is

$$
N \prod_{p \mid N}\left(1+\frac{1}{p}\right)
$$

Hence if $N$ is square-free, all variants of $\mathfrak{s l}_{N}$ gauge theories can be found as images of $S U(N)$ gauge theory under some gauge transformation. If not, there are separate duality orbits.

9.3.1. Example: $S L_{3}$. The Lie algebra $\mathfrak{s l}_{3}$ gives an example with no subtleties. The standard $S U(3)$ gauge theory, the standard $P S U(3)_{0}$ gauge theory and the two T-images $P S U(3)_{1,2}$ exhaust the possible variants, even keeping track of the spin structure dependence. They are all in the same duality orbit, with $S$ exchanging $P S U(3)_{1}$ and $P S U(3)_{2}$.

9.3.2. Example: $S L_{4}$. This is a much more intricate example. Ignoring spin subtleties, there are 7 variants of the gauge theory, but the main duality orbit only includes 6 of them.

Starting from $S U(4)$, which has line defects of charges $(0, e), e \in \mathbb{Z}_{4}$ and is $T$-invariant, $S$-duality gives $\left(S U(4) / \mathbb{Z}_{4}\right)_{0}$, which has line defects of charges $(m, 0), m \in \mathbb{Z}_{4}$. There are three more variants produced by the action of $T,\left(S U(4) / \mathbb{Z}_{4}\right)_{i}$ with $i=1,2,3$. The $\left(S U(4) / \mathbb{Z}_{4}\right)_{1,3}$ are exchanged by $S$, but $\left(S U(4) / \mathbb{Z}_{4}\right)_{2}$ is mapped to a modified version of $S U(4) / \mathbb{Z}_{2}$, which we denote by $\left(S U(4) / \mathbb{Z}_{2}\right)_{1}=S O(6)_{1}$. On the other hand, the standard $\left(S U(4) / \mathbb{Z}_{2}\right)_{0}=S O(6)_{0}$ is invariant under both $S$ and $T$.

If we keep track of spin subtleties, the situation should become even richer. The Nahm pole for $S U(4)$ requires a spin structure, suggesting that its $S$-dual gauge theory's Neumann boundary condition should require a spin structure as well. Indeed, the periodicity of the 
$\theta$ angle in $S U(4) / \mathbb{Z}_{4}$ is twice as long on non-spin manifolds, and $\left(S U(4) / \mathbb{Z}_{4}\right)_{4}$ is a good candidate for the S-dual of $S U(4)$. We expect the $S$-dual of $\left(S U(4) / \mathbb{Z}_{4}\right)_{0}$ to be Spin- $S U(4)$.

Because of the identity $S T S=T^{-1} S T^{-1}$ applied to $S U(4)$, we get that $S$ maps $\left(S U(4) / \mathbb{Z}_{4}\right)_{3}$ to $\left(S U(4) / \mathbb{Z}_{4}\right)_{5}$. Similarly, $S$ maps $\left(S U(4) / \mathbb{Z}_{4}\right)_{1}$ to $\left(S U(4) / \mathbb{Z}_{4}\right)_{7}$.

The $T$ operation does not fix $S U(4) / \mathbb{Z}_{2}=S O(6)$ anymore, but $T^{2}$ does. We also have Spin- $S O(6)$ versions of the gauge theory. It would be nice to fill in the remaining structure of the duality groupoid.

\section{OThER DUALITY KERNELS}

In this section, we discuss another family of kernel vertex algebras $Y_{\kappa}(G)$ associated to the $D_{0,1} \rightarrow D_{1,0}$ junction and thus to the standard qGL duality, in which conformal dimensions away from the vacuum are strictly positive.

In the case when $G$ is a reductive Lie group that is Langlands self-dual (for instance, $E_{8}$ or $\left.G L_{n}\right)$ the vertex algebras $Y_{\kappa}(G)$ were introduced in [36] and [16], as the result of a $D_{0,1} \rightarrow N_{1,-1} \rightarrow D_{1,0}$ composition of junctions. The objective of this section is to generalize that construction for an arbitrary simple Lie group $G$ and discuss the corresponding qGL duality functors.

The generalization has two levels of complexity. For simply-laced groups, it turns out that the simplest generalization of the above construction involves the extended duality groupoid: we can build analogous $D_{0,1} \rightarrow D_{1,0}$ junctions for the generalized gauge theory $G_{-1}$ which is the $T^{-1}$ image of the gauge theory associated to the adjoint form of the gauge group $G$.

Notice that the $G_{-1}$ theory is mapped by $\mathrm{S}$ to the $T$ image of $G$, aka $G_{1}$. Indeed, $S \circ G_{-1}=S T^{-1} \circ G=T(S T S) \circ G=G_{1}$. On the other hand, the $R S$ transformation maps the $G_{-1}$ theory to itself.

The construction of $Y_{\kappa}(G)$ is simpler than the one given in the Section 5 in that for general $\kappa$ there is only one direct sum over dominant integral weights (rather than two). The vertex algebra $Y_{\kappa}(G)$ comes equipped with the action of two copies of $\widehat{\mathfrak{g}}=\widehat{L_{\mathfrak{g}}}$, of levels $\kappa+1$ and $\kappa^{-1}+1$.

In the case that $G$ is self-dual and simply-connected (which for simple Lie groups means $G=E_{8}$ ), we have already described this vertex algebra and the corresponding kernel the qGL duality TST in Section 4.4. Tensoring this kernel with the line bundle $\mathcal{L}_{G}^{-1}$ on each factor $\operatorname{Bun}_{G}$, we should then obtain the kernel of the main qGL duality $S$. For other simply-laced simple Lie groups the construction is identical, but tensoring with $\mathcal{L}_{G}^{-1}$ maps the kernel to a gerbe-twisted version of $\mathrm{Bun}_{G}$.

The action on $S$ on other nodes of the duality groupoid requires appropriate modifications of the vertex algebra $Y_{\kappa}(G)$. Based on the examples we treat below in detail, we expect that the modifications are always finite index extensions of $Y_{\kappa}(G) \otimes A$ for some rational vertex algebra $A$ that depends sensitively on the choice of gauge groups and discrete $\theta$-angles.

We then generalize $Y_{\kappa}(G)$ to non-simply laced simple Lie groups. In this case, $Y_{\kappa}(G)$ is larger: it involves two summations over dominant weights for groups with the lacing number $m=2$ and four for $G_{2}$, which has lacing number $m=3$. 
10.1. Construction of $Y_{\kappa}(G)$ for simply-laced $G$. Let's recall and extend the construction in the self-dual case (see [36, 16] and Section 4.4 above). Take $G$ to be the adjoint form of the group, so that ${ }^{L} G$ is simply-connected.

Consider the following composition of junctions

$$
D_{0,1} \rightarrow N_{1,-1} \rightarrow D_{1,0}
$$

in the bulk theory $\mathcal{T}_{\kappa}^{G-1} \equiv T^{-1} \circ \mathcal{T}_{\kappa+1}^{G}$.

The first junction is the $T^{-1}$ image of $D_{0,1} \rightarrow N_{1,0}$ in $\mathcal{T}_{\kappa+1}^{G}$. The corresponding junction vertex algebra is $\widehat{\mathfrak{g}}$ of level $\kappa+1$.

The second junction is the $R S T^{-1}$ image of $D_{0,1} \rightarrow N_{1,0}$ in $\mathcal{T}_{\kappa^{-1}+1}^{G}$. The corresponding junction vertex algebra is $\widehat{\mathfrak{g}}$ of level $\kappa^{-1}+1$.

The composition of the two junctions gives an extension

$$
Y_{\kappa}\left(G_{-1}\right)=\bigoplus_{\lambda \in P^{+}} \mathbb{V}_{\lambda, \kappa+1} \otimes \mathbb{V}_{\lambda^{*}, \kappa^{-1}+1},
$$

where $\lambda^{*}$ was defined in Section 5.3 . Notice that $\lambda$ and $\lambda^{*}$ belong to the root lattice and the vertex algebra has two $\widehat{\mathfrak{g}}-$ and $G[[z]]$-actions.

Applying the localization functor to it, we therefore obtain a twisted $D$-module $\Delta_{\kappa}^{T S T}(G)$ on $\operatorname{Bun}_{G} \times \operatorname{Bun}_{G}$, with the twists $\kappa+1$ and $\kappa^{-1}+1$ along the two factors, which we expect to be a kernel of the functor

$$
\varepsilon_{-\kappa-1}^{G, T S T}: D_{-\kappa-1}\left(\operatorname{Bun}_{G}\right) \rightarrow D_{\kappa^{-1}+1}\left(\operatorname{Bun}_{G}\right)
$$

corresponding to the qGL duality $T S T=T S_{1} T$. Since the action of $T$ on the category of $\kappa$-twisted $D$-modules corresponds to tensoring with the line bundle $\mathcal{L}_{G}$, the kernel of the qGL duality $S$ can now also be constructed: we simply take the tensor product

$$
\Delta_{\kappa}^{T S T}(G) \otimes\left(\mathcal{L}_{G}^{-1} \otimes \mathcal{L}_{G}^{-1}\right) .
$$

This a now gerbe-twisted $D$-module on $\operatorname{Bun}_{G} \times \operatorname{Bun}_{G}$ with the twists $\kappa$ and $\kappa^{-1}$ along the two factors, which should give rise to the kernel

$$
\mathcal{E}_{-\kappa}^{G, S}: \mathcal{G}_{1}-D_{-\kappa}\left(\operatorname{Bun}_{G}\right) \rightarrow \mathcal{G}_{-1}-D_{\kappa^{-1}}\left(\operatorname{Bun}_{G}\right) .
$$

where $\mathcal{G}_{ \pm 1}$ is the $\mathbb{Z}_{n(G)}$-gerbe of $n(G)$ th roots of $\mathcal{L}_{G}^{\otimes \pm 1}$.

For other choices of generalized gauge group, different from $G_{-1}^{\text {Adj }}$, the procedure above can still be implemented, but we need to identify appropriate junctions $D_{0,1} \rightarrow N_{1,-1}$ for $G$ and ${ }^{L} G$.

Based on general gauge theory considerations and past examples, we expect the existence of junctions to support vertex algebras which include two copies of $\widehat{\mathfrak{g}}$ as well as an auxiliary rational vertex algebras, which are needed to implement correct functors from $\mathcal{C}_{\kappa}^{G}\left(N_{1,-1}\right)$. The composition of such junctions should give a finite index extension of $Y_{\kappa}\left(G_{-1}^{\mathrm{Adj}}\right) \otimes A$ for some rational vertex algebra $A$.

It is far from obvious, though, that our strategy of composing simpler junctions in order to produce, say, a $D_{0,1} \rightarrow N_{1,-1}$ junction will give vertex algebras of this form, as we can see from the example in this remark. 
Remark 10.1. Suppose that we start with the composition of junctions

$$
D_{0,1} \rightarrow N_{1,-1} \rightarrow D_{1,0}
$$

in the bulk theory $\mathcal{T}_{\kappa}^{G}$ rather than $\mathcal{T}_{\kappa}^{G-1}$ as we did before. Then we can represent the second junction $N_{1,-1} \rightarrow D_{1,0}$ as the $S R T^{-1}$ image of junction $D_{0,1} \rightarrow N_{1,0}$ in $\mathcal{T}_{\kappa^{-1}+1}^{L_{G}}$ because $T^{-1}$ is a legitimate duality of $\mathcal{T}_{\kappa^{-1}+1}^{L}$ (recall that ${ }^{L} G$ is assumed to be simply-connected). But we can no longer represent the first junction as the $T^{-1}$ image of $D_{0,1} \rightarrow N_{1,0}$ in $\mathcal{T}_{\kappa+1}^{G}$ because $T^{-1}$ is not a legitimate duality there.

Instead, we can try to build it as a composition of two junctions. The simplest one is

$$
D_{0,1} \rightarrow N_{1,0} \rightarrow N_{1,-1}
$$

(again, in the bulk theory $\mathcal{T}_{\kappa}^{G}$ ), where the second junction is the $S T$ image of the basic junction $N_{0,1} \rightarrow N_{1,0}$ in the bulk theory $\mathcal{T}_{-(\kappa+1) / \kappa}^{L}$. The vertex algebra corresponding to (10.3) is therefore, for irrational $\kappa$,

$$
\bigoplus_{\lambda \in P_{\text {ad }}^{+}} \mathbb{V}_{\lambda, \kappa} \otimes M_{\left(\lambda^{*}, 0\right),-\kappa /(\kappa+1)}
$$

where we use the notation 3.12 . Hence the vertex algebra of the junction 10.2 is, for irrational $\kappa$,

$$
\bigoplus_{\lambda \in P_{\mathrm{ad}}^{+}, \mu^{\vee} \in P^{+}} \mathbb{V}_{\lambda, \kappa} \otimes M_{\left(\lambda^{*}, \mu^{\vee}\right),-\kappa /(\kappa+1)} \otimes \mathbb{V}_{\mu^{\vee *}, \kappa^{-1}+1}
$$

Unfortunately, this vertex algebra has unbounded conformal dimensions. That's why we prefer $Y_{\kappa}(G)$ which does not suffer from this defect.

10.2. Kernels for $S L_{2}$. In Section 8 we encountered the vertex algebra d $(2,1,-\kappa)_{1}$, which enters in slightly different guises in the construction of duality kernels for a variety of qGL dualities involving $S U(2)$ and $S O(3)$. According to the definition above, $Y_{\kappa}\left(S O(3)_{1}\right) \equiv$ $\frac{\mathrm{d}(2,1,-\kappa)_{1}}{\mathrm{su}(2)_{1}}$.

Ignoring spin subtleties, but keeping track of the full groupoid with nodes $S U(2), S O(3)_{0}$ and $S O(3)_{1} \equiv S O(3)_{-1}$, we summarize the appearances of $\mathrm{d}(2,1,-\kappa)_{1}$ :

(i) The affine subalgebra $\widehat{\mathfrak{s l}}(2)_{\kappa+1} \times \widehat{\mathfrak{s l}}(2)_{\kappa^{-1}+2}$ of $\mathrm{d}(2,1,-\kappa)_{1}$ can be used to associate (via the localization functor) to the vertex algebra $\mathrm{d}(2,1,-\kappa)_{1}$ a twisted $D$-module on $\operatorname{Bun}_{S L_{2}} \times \operatorname{Bun}_{P S L_{2}}$ with the twists $\kappa+1$ along the first factor and $\kappa^{-1}+2$ along the second factor. Then, tensoring with the inverse of the generating line bundle $\mathcal{L}_{S L_{2}}$ along the first factor and the inverse of the generating line bundle $\mathcal{L}_{P S L_{2}}$ along the second factor, we obtain a $\left(\kappa, \kappa^{-1}\right)$-twisted $D$-module on $\operatorname{Bun}_{S L_{2}} \times \operatorname{Bun}_{P S L_{2}}$. This is our candidate for the kernel of the standard qGL duality associated to the $S$ operation $S U(2) \rightarrow S O(3)_{0}$.

(ii) The coset $\frac{\mathrm{d}(2,1,-\kappa)_{1}}{\mathrm{su}(2)_{1}}$ allows conformal blocks to be defined as twisted $D$-modules on $\operatorname{Bun}_{P S L_{2}} \times \operatorname{Bun}_{P S L_{2}}$ with the twist $\kappa+1$ along the first factor and the $\kappa^{-1}+1$ along the second factor. This is our candidate for the kernel of a modified qGL duality associated to the $S$ operation $S O(3)_{1} \rightarrow S O(3)_{1}$. 
In particular, we can naturally define $Y_{\kappa}\left(S O(3)_{0}\right)=Y_{\kappa}(S U(2))=\mathrm{d}(2,1,-\kappa)_{1}$, with appropriate choices of currents to couple to $S L(2)$ and $P S L(2)$ bundles.

Spin subtleties manifest themselves in a rather straight-forward manner. The spinextended groupoid has nodes $S U(2)_{b}, S U(2)_{s}, S O(3)_{0}, S O(3)_{1}, S O(3)_{2}$ and $S O(3)_{3} \equiv S O(3)_{-1}$.

(i) In the above statement (i), tensoring with $\mathcal{L}_{P S L_{2}}^{-1}$ actually maps $\left(\kappa^{-1}+2\right)$-twisted $D$ modules on $\operatorname{Bun}_{P S L_{2}}$ to $\kappa^{-1}$-twisted and $\mathcal{G}$-twisted $D$-modules on Bun $P S L_{2}$. Thus, the resulting kernel should correspond to the qGL duality $S: S U(2)_{b} \rightarrow S O(3)_{2}$.

(ii) A different vertex algebra is required for the qGL duality $S U(2)_{s} \rightarrow S O(3)_{0}$. A likely candidate is the even part of $\mathrm{d}(2,1,-\kappa)_{1} \times \mathrm{so}(3)_{1}$. This vertex algebra has an affine $\widehat{\mathfrak{s} l}(2)_{\kappa+1} \times \widehat{\mathfrak{s l}}(2)_{\kappa^{-1}+4}$ subalgebra and the spin and $\widehat{\mathfrak{s} l}(2)_{\kappa+1}$ weight of fields are tied together. This means that its localization functor yields $\kappa$-twisted $D$-modules along Bun $_{S p i n-S L_{2}}$ and $\kappa^{-1}$-twisted $D$-modules along $\operatorname{Bun}_{P S L_{2}}$.

(iii) The $\frac{\mathrm{d}(2,1,-\kappa)_{1}}{\mathrm{su}(2)_{1}}$ vertex algebra is the natural candidate for the qGL duality $S O(3)_{3} \rightarrow$ $S O(3)_{1}$.

(iv) Likewise, a kernel for the qGL duality $S O(3)_{1} \rightarrow S O(3)_{3}$ would require a vertex algebra with an $\widehat{\mathfrak{s l}}(2)_{\kappa+3} \times \widehat{\mathfrak{s l}}(2)_{\kappa^{-1}+3}$ sub-algebra. A possible solution is to combine $\frac{\mathrm{d}(2,1,-\kappa)_{1}}{\mathrm{su}(2)_{1}}$ with the even part of $\mathrm{so}(3)_{1} \times \mathrm{so}(3)_{1}$.

10.3. Kernels for $S L_{3}$. This is a particularly straightforward example. It has no spin subtleties, but it illustrates the new challenges which arise when we go beyond $S L_{2}$.

We have learned above how to build a $D_{0,1} \rightarrow D_{1,0}$ junction in the $P S U(3)_{2} \equiv P S U(3)_{-1}$ generalized gauge theory. It supports the extension of $\widehat{\mathfrak{s l}}(3)_{\kappa+1} \times \widehat{\mathfrak{s l}}(3)_{\kappa^{-1}+1}$ we denote as $Y_{\kappa}\left(P S U(3)_{2}\right)$.

The next simplest junction we can produce is a $D_{0,1} \rightarrow D_{1,0}$ junction in the $P S L(3)_{1}$ generalized gauge theory, the $T$ image of the standard $P S L(3)$ theory.

As an intermediate step, we can decompose the junction as $D_{0,1} \rightarrow N_{1,-2} \rightarrow D_{1,0}$ and associate $D_{0,1} \rightarrow N_{1,-2}$ to a $\widehat{\mathfrak{s l}}(3)_{\kappa+2}$ Kac-Moody algebra. We are left with the problem of building $N_{1,-2} \rightarrow D_{1,0}$.

A similar, $R S$ dual decomposition $N_{1,-2} \rightarrow N_{2,-1} \rightarrow D_{1,0}$ gives an $\widehat{\mathfrak{s l}}(3)_{\kappa^{-1}+2}$ factor, but still leaves a non-trivial problem: build an $N_{1,-2} \rightarrow N_{2,-1}$ junction in the $P S U(3)_{1}$ theory.

We can solve the problem with one final step: the composition $N_{1,-2} \rightarrow N_{1,-1} \rightarrow N_{2,-1}$ in the $P S U(3)_{1}$ theory. Indeed, both partial junctions can be dualized to $N_{1,0} \rightarrow N_{1,1}$ in the standard $P S U(3)$ theory, i.e. $N_{0,1} \rightarrow N_{1,-1}$ in the $S U(3)$ theory.

Keeping track of couplings, that gives a $\mathcal{W}_{\frac{\kappa+1}{\kappa+2}}$ and a $\mathcal{W}_{\frac{\kappa+1}{2 \kappa+1}}$ algebras. We can then go back to the original junction and associate differently. The $D_{0,1} \rightarrow N_{1,-2} \rightarrow N_{1,-1}$ composition in the $P S L(3)_{1}$ theory can be recognized by the GKO coset construction of $\mathcal{W}$ as supporting $\widehat{\mathfrak{s l}}(3)_{\kappa+1} \times \mathrm{su}(3)_{1}$, possibly coupled to $P S L(3)$ bundles through the total level $\kappa+2$ currents.

Overall, we obtained a junction $D_{0,1} \rightarrow D_{1,0}$ in the $P S U(3)_{1}$ gauge theory supporting an extension of

$$
\widehat{\mathfrak{s l}}(3)_{\kappa+1} \times \widehat{\mathfrak{s l}}(3)_{\kappa^{-1}+1} \times \mathrm{su}(3)_{1} \times \mathrm{su}(3)_{1}
$$


we denote as $Y_{\kappa}\left(P S U(3)_{1}\right)$. The extension involves the category of lines on $N_{1,-1}$ in the $P S U(3)_{1}$ theory, i.e. the category of lines on on $N_{1,-1}$ in the standard $S U(3)$ gauge theory: $\mathrm{KL}_{\frac{\kappa+1}{\kappa+2}}\left(\mathfrak{s} l_{3}\right)$. In particular, it involves a sum over all highest weights.

Conformal blocks of such vertex algebra give a potential $S$ kernel for the category of twisted $D$-modules associated to the $P S L(3)_{1}$ theory.

It is very tempting to assume that the same vertex algebra may also occur at a junction $D_{0,1} \rightarrow D_{1,0}$ in the standard $S U(3)$ or $P S U(3)$ gauge theories. Indeed, it has an $\widehat{\mathfrak{s l}}(3)_{\kappa+1} \times$ $\widehat{\mathfrak{s l}}(3)_{\kappa^{-1}+3}$ sub-algebra and it may be possible to couple it to $S L_{3} \times P S L_{3}$ bundles to produce $D$-modules with the correct twists.

We cannot easily confirm this naive expectation by composing basic junctions. With a small extra conjecture, we can get a somewhat similar statement, but involving an extra $\mathrm{su}(3)$ spectator factor. We have derived $\widehat{\mathfrak{s l}}(3)_{\kappa+1} \times \mathrm{su}(3)_{1}$ at $D_{0,1} \rightarrow N_{1,-2} \rightarrow N_{1,-1}$ in the $\operatorname{PSL}(3)_{1}$ theory. We can look as a similar composition: $N_{0,1} \rightarrow N_{1,-2} \rightarrow N_{1,-1}$ in the $P S L(3)_{1}$ theory.

In analogy with the $S U(2)$ case, we conjecture that, up to a re-definition of the stress tensor, there is an $N_{0,1} \rightarrow N_{1,-1}$ junction in the $P S L(3)_{1}$ theory which supports $\mathcal{W}_{\kappa+1} \times$ $\mathrm{su}(3)_{1}$. Equivalently, we conjecture that there is an $N_{0,1} \rightarrow N_{1,0}$ junction in the $P S L(3)_{2}$ theory which supports $\mathcal{W}_{\kappa} \times \operatorname{su}(3)_{1}$.

If this conjecture is correct, then a direct construction of $D_{0,1} \rightarrow D_{1,0}$ in the standard $P S U(3)_{0}$ gauge theory can proceed as follows. We can first decompose it to $D_{0,1} \rightarrow N_{1,-2} \rightarrow$ $N_{1,-1} \rightarrow D_{1,0}$. The $D_{0,1} \rightarrow N_{1,-2}$ junction can be produced from $D_{0,1} \rightarrow N_{1,-3} \rightarrow N_{1,-2}$ : $N_{1,-3} \rightarrow N_{1,-2}$ in $P S U(3)_{0}$ is dual to $N_{1,0} \rightarrow N_{1,1}$ in $P S U(3)_{0}$ and thus to $N_{0,1} \rightarrow N_{1,-1}$ in $S U(3)$ and supports $\mathcal{W}_{\frac{\kappa+2}{\kappa+3}}$. It can combine with $\widehat{\mathfrak{s l}}(3)_{\kappa+3}$ to give $\widehat{\mathfrak{s l}}(3)_{\kappa+2} \times \mathrm{su}(3)_{1}$ at the $D_{0,1} \rightarrow N_{1,-2}$ junction.

Next, we map $N_{1,-2} \rightarrow N_{1,-1}$ in $P S U(3)_{0}$ to $N_{1,-1} \rightarrow N_{1,0}$ in $P S U(3)_{1}$, to $N_{0,1} \rightarrow N_{1,-1}$ in $P S U(3)_{1}$, to $N_{0,1} \rightarrow N_{1,0}$ in $P S U(3)_{2}$. Then the junction should support $\mathcal{W}_{\frac{\kappa+2}{\kappa+1}} \times \operatorname{su}(3)_{1}$.

Assembling the pieces, we get an extension of $\widehat{\mathfrak{s l}}(3)_{\kappa+2} \times \mathrm{su}(3)_{1} \times \mathcal{W}_{\frac{\kappa+2}{\kappa+1}} \times \mathrm{su}(3)_{1}$ at $D_{0,1} \rightarrow N_{1,-1}$ in $P S U(3)_{0}$, which we can presumably be identified with something like $\widehat{\mathfrak{s l}}(3)_{\kappa+1} \times \mathrm{su}(3)_{1} \times \mathrm{su}(3)_{1} \times \mathrm{su}(3)_{1}$. If this is correct, the result will be some extension of

$$
\widehat{\mathfrak{s l}}(3)_{\kappa+1} \times \widehat{\mathfrak{s l}}(3)_{\kappa^{-1}+1} \times \mathrm{su}(3)_{1} \times \mathrm{su}(3)_{1} \times \mathrm{su}(3)_{1}
$$

It would be interesting to test this conjecture further. We leave that to future work.

10.4. $S p(N)$ and $S O(2 N+1)$ and other non-simply laced groups. The basic challenge here is that, unlike the simply-laced case, $N_{1,-1}$ is not self-dual under the basic duality $R S_{m}$. Rather, it is mapped by $R S_{m}$ to $N_{m,-1} \neq N_{1,-1}$ if the lacing number $m>1$. This means that if we want to use the composition $D_{0,1} \rightarrow N_{1,-1} \rightarrow D_{1,0}$ for a non-simply laced group $G$, we need to describe the vertex algebra for the junction $N_{1,-1} \rightarrow D_{1,0}$ for the coupling $\kappa$ and group $G$. Hence, we have to describe its $R S_{m}$-dual: $D_{0,1} \rightarrow N_{m,-1}$ for the coupling $(m \kappa)^{-1}$ and group ${ }^{L} G$.

In what follows, we will pretend that $T$ is a legitimate duality for both $G$ and ${ }^{L} G$. We will simply ignore here subtleties concerning the global form of the group. 
Consider first the case $m=2$. Then we can decompose further the latter junction as $D_{0,1} \rightarrow N_{1,-1} \rightarrow N_{2,-1}$. The $N_{1,-1} \rightarrow N_{2,-1}$ junction for the coupling $(2 \kappa)^{-1}$ and group ${ }^{L} G$ is the same as $N_{1,0} \rightarrow N_{2,1}$ at $(2 \kappa)^{-1}+1$ and group ${ }^{L} G$, which is the same as $N_{0,1} \rightarrow N_{1,-1}$ at $-\frac{\kappa}{1+2 \kappa}$ and the original group $G$. In turn, that is the same as $N_{0,1} \rightarrow N_{1,0}$ at $\frac{\kappa+1}{2 \kappa+1}$ and group $G$.

On the other hand, $D_{0,1} \rightarrow N_{1,-1}$ at $(2 \kappa)^{-1}$ and group ${ }^{L} G$ is the same as $D_{0,1} \rightarrow N_{1,0}$ at $(2 \kappa)^{-1}+1$ and group ${ }^{L} G$.

Thus, the resulting kernel vertex algebra $Y_{\kappa}(G)$ is a double extension of $\mathfrak{g}_{\kappa+1} \times \mathcal{W}_{\frac{\kappa+1}{2 \kappa+1}}(\mathfrak{g}) \times$ $\widehat{L}_{\mathfrak{g}_{(2 \kappa)^{-1}+1}}$. For irrational $\kappa$, it can be written explicitly as a double direct sum, as in Section 5. One can show that this vertex algebra has non-negative conformal dimensions. When we apply the localization functor to the vertex algebra $Y_{\kappa}(G)$, we should obtain a kernel in a suitable version of the category of twisted $D$-modules on $\operatorname{Bun}_{G} \times \operatorname{Bun}_{L_{G}}$ with the twists $\kappa+1$ along the first factor and $(2 \kappa)^{-1}+1$ along the second factor. This $D$-module should give rise to the qGL duality $T S T$.

When $\mathfrak{g}=\mathfrak{s o}(2 n+1)$, string theory suggests that the extension $\mathcal{W}_{\frac{\kappa+1}{2 \kappa+1}}(\mathfrak{s o}(2 n+1)) \times$ $\widehat{\mathfrak{s p}}(n)_{(2 \kappa)^{-1}+1}$ should be identified with $\widehat{\mathfrak{o s p}}(1 \mid n)_{\kappa^{-1}+1}$ and hence the kernel vertex algebra is an extension of $\widehat{\mathfrak{s o}}(2 n+1)_{\kappa+1} \times \widehat{\mathfrak{o s p}}(1 \mid n)_{\kappa^{-1}+1}$.

With a bit more care, and perhaps extra factors of so $(2 n+1)$ and $\operatorname{sp}(2 n)$ WZW models at level 1, we should be able to manufacture kernels adapted to specific pairs of gauge groups and discrete $\theta$-angles. Similar considerations should apply to other groups of lacing number $m=2$.

For lacing number $m=3$, we need to work harder.

If we attempt a decomposition $D_{0,1} \rightarrow N_{2,-1} \rightarrow N_{3,-1}$, the $N_{2,-1} \rightarrow N_{3,-1}$ junction is easy: it is dual to $N_{1,-1} \rightarrow N_{3,-2}$, which is dual to $N_{1,0} \rightarrow N_{3,1}$, dual to $N_{0,1} \rightarrow N_{1,1}$ and hence finally to $N_{0,1} \rightarrow N_{1,0}$.

On the other hand, $D_{0,1} \rightarrow N_{2,-1}$ requires a further decomposition, such as $D_{0,1} \rightarrow$ $N_{1,-1} \rightarrow N_{2,-1}$.

Still, $N_{1,-1} \rightarrow N_{2,-1}$ is not an elementary junction. We can attempt a further decomposition $N_{1,-1} \rightarrow N_{3,-2} \rightarrow N_{2,-1}$. The first half is dual to $N_{1,0} \rightarrow N_{3,1}$ and thus to $N_{0,1} \rightarrow N_{1,-1}$, which is elementary. The second half is dual to $N_{3,1} \rightarrow N_{2,1}$ and then to $N_{1,-1} \rightarrow N_{3,-2}$, which we just analyzed.

Thus a potential kernel vertex algebra for lacing number $m=3$ will be a quadruple extension of the rough form $\widehat{\mathfrak{g}}_{\kappa+1} \times \mathcal{W}_{\kappa^{\prime}}(\mathfrak{g}) \times \mathcal{W}_{\kappa^{\prime \prime}}(\mathfrak{g}) \times \mathcal{W}_{\kappa^{\prime \prime \prime}}(\mathfrak{g}) \times \widehat{\mathfrak{g}}_{(3 \kappa)^{-1}+1}$.

We leave to future work checks of positivity of conformal dimensions for this extension.

\section{Some Future Directions}

This work leaves open a variety of questions in gauge theory, vertex algebras and the Geometric Langlands Program. Here we list some of the most important ones.

- Rational $\kappa$ : What are the junctions associated to the extra boundary conditions which only exist at rational values of $\kappa$ ?

Do sheaves of coinvariants of the corresponding junction vertex algebras give rise to dual pairs of objects? 
What is the best way to describe the limit of the kernel vertex algebras constructed in this paper when $\kappa$ tends to a rational value?

What are the limits of the corresponding qGL duality functors?

Do issues of temperedness affect equally all possible kernels obtained from the junctions, or different kernels have a different domain of effectiveness?

- Same questions at the critical level. Furthermore, the theory of coinvariants twisted by flat bundles needs to be developed mathematically.

- What is the full spin-refined duality groupoid?

- Can auxiliary rational vertex algebras be found, which can combine with $\mathcal{W}$ - or Kac-Moody algebras to give elementary junctions for all possible global forms of the gauge group?

- Is there some simple criterion which guarantees or forbids the existence of junction vertex algebras with positive conformal dimensions?

\section{REFERENCES}

[1] M. Aganagic, E. Frenkel, and A. Okounkov, Quantum q-Langlands Correspondence, Trans. Moscow Math. Soc. 79 (2018) 1-83, arXiv:1701.03146

[2] L.F. Alday, D. Gaiotto, and Y. Tachikawa, Liouville Correlation Functions from Four-dimensional Gauge Theories, Lett. Math. Phys. 91 (2010) 167-197, arXiv:0906.3219.

[3] T. Arakawa, Representation Theory of Superconformal Algebras and the Kac-Roan-Wakimoto Conjecture, Duke Math. J. 130 (2005) 435-478.

[4] T. Arakawa, Representation theory of W-algebras, Invent. Math. 169 (2007) 219-320.

[5] T. Arakawa and E. Frenkel, Quantum Langlands Duality of Representations of W-algebras, to appear in Compositio Math., arXiv:1807.01536

[6] D. Arinkin and D. Gaitsgory, Singular support of coherent sheaves and the geometric Langlands conjecture, Selecta Math. 21 (2015) 1-199.

[7] S. Arkhipov and D. Gaitsgory, Differential operators on the loop group via chiral algebras IMRN (2002) 165-210, arXiv:math/0009007.

[8] O. Aharony, N. Seiberg, and Y. Tachikawa, Reading between the lines of four-dimensional gauge theories, JHEP 1308 (2013) 115, arXiv:1305.0318.

[9] A. Balasubramanian and J. Teschner, Supersymmetric field theories and geometric Langlands: The other side of the coin, arXiv:1702.06499

[10] A. Beilinson, Langlands parameters for Heisenberg modules, arXiv:math/0204020.

[11] A. Beilinson and V. Drinfeld, Quantization of Hitchin's integrable system and Hecke eigensheaves, http://www.math.uchicago.edu/ drinfeld/langlands/QuantizationHitchin.pdf

[12] A. Beilinson and V. Drinfeld, Chiral algebras, Colloq. Publ. 51, AMS, 2004.

[13] D. Beraldo, Loop group actions on categories and Whittaker invariants, Adv. in Math. 322 (2017) 565-636, arXiv:1310.5127.

[14] D. Butson and P. Yoo, Degenerate Classical Field Theories and Boundary Theories, arXiv:1611.00311.

[15] K. Costello and D. Gaiotto, Vertex Operator Algebras and 3d N=4 gauge theories, arXiv:1804.06460.

[16] T. Creutzig and D. Gaiotto, Vertex Algebras for S-duality, arXiv:1708.00875

[17] R. Dijkgraaf and E. Witten, Topological gauge theories and group cohomology, Comm. Math. Phys. 129 (1990) 393-429.

[18] V. Drinfeld and D. Gaitsgory, Compact generation of the category of D-modules on the stack of $G$-bundles on a curve, arXiv:1112.2402.

[19] C. Elliott and P. Yoo, Geometric Langlands Twists of $N=4$ Gauge Theory from Derived Algebraic Geometry, arXiv:1507.03048 
[20] C. Elliott and P. Yoo, A Physical Origin for Singular Support Conditions in Geometric Langlands Theory, arXiv:1707.01292.

[21] P. Etingof, S. Gelaki, D. Nikshych, and V. Ostrik, Tensor Categories, AMS 2015.

[22] B. Feigin and E. Frenkel, Affine Kac-Moody algebras at the critical level and Gelfand-Dikii algebras, in Infinite Analysis, eds. A. Tsuchiya, T. Eguchi, M. Jimbo, Adv. Ser. in Math. Phys. 16, 197-215, Singapore: World Scientific, 1992.

[23] E. Frenkel, Affine Algebras, Langlands Duality and Bethe Ansatz, in Proceedings of the International Congress of Mathematical Physics, Paris, 1994, ed. D. Iagolnitzer, pp. 606-642, International Press, 1995, arXiv:q-alg/9506003.

[24] E. Frenkel, Wakimoto modules, opers and the center at the critical level, Adv. Math. 195 (2005) 297-404, arXiv:math/0210029

[25] E. Frenkel, Lectures on the Langlands Program and Conformal Field Theory, in Frontiers in Number Theory, Physics and Geometry II, eds. P. Cartier, e.a., pp. 387-536, Springer, 2007, arXiv:hepth/0512172

[26] E. Frenkel, Gauge theory and Langlands duality, Séminaire Bourbaki, Astérisque 332 (2010) 369-403, arXiv:0906.2747.

[27] E. Frenkel, Langlands Program, Trace Formulas, and their Geometrization, Bull. AMS 50 (2013) 1-55, arXiv:1202.2110.

[28] E. Frenkel and D. Ben-Zvi, Vertex Algebras and Algebraic Curves, Mathematical Surveys and Monographs 88, Second Edition, AMS, 2004.

[29] E. Frenkel and D. Gaitsgory, D-modules on the affine Grassmannian and representations of affine Kac-Moody algebras, Duke Math. J. 125 (2004) 279-327.

[30] E. Frenkel and D. Gaitsgory, Affine Kac-Moody algebras and local geometric Langlands correspondence, in Algebraic Geometry and Number Theory, Progress in Math. 253, pp. 69-260, Birkhäuser Boston, 2006, math.RT/0508382.

[31] E. Frenkel, D. Gaitsgory, and K. Vilonen, Whittaker patterns in the geometry of moduli spaces of bundles on curves, Annals of Math. 153 (2001) 699-748.

[32] E. Frenkel, S. Gukov, and J. Teschner, Surface Operators and Separation of Variables, JHEP (2016) 179, arXiv: 1506.07508 .

[33] E. Frenkel, V. Kac, and M. Wakimoto, Characters and fusion rules of W-algebras via quantized Drinfeld-Sokolov reduction, Comm. Math. Phys. 147 (1992) 295-328.

[34] E. Frenkel and E. Witten, Geometric endoscopy and mirror symmetry, Commun. Num. Theor. Phys. 2 (2008) 113-283, arXiv:0710.5939

[35] E. Frenkel and E. Witten, On the brane interpretation of conformal blocks, unpublished manuscript (2010).

[36] D. Gaiotto, S-duality of boundary conditions and the Geometric Langlands program, arXiv:1609.09030

[37] D. Gaiotto, Twisted compactifications of $3 d N=4$ theories and conformal blocks, arXiv:1611.01528.

[38] D.Gaiotto, G.W.Moore and A.Neitzke, Framed BPS States, Adv. Theor. Math. Phys. 17 (2013) 241, arXiv: 1006.0146

[39] D. Gaiotto and M. Rapcak, Vertex Algebras at the Corner, Preprint arXiv:1703.00982

[40] D. Gaiotto and E. Witten, Supersymmetric Boundary Conditions in N $=4$ Super Yang-Mills Theory, arXiv:0804.2902.

[41] D. Gaiotto and E. Witten, S-Duality of Boundary Conditions In $N=4$ Super Yang-Mills Theory, arXiv:0807.3720

[42] D. Gaiotto and E. Witten, Knot Invariants from Four-Dimensional Gauge Theory, arXiv:1106.4789.

[43] D. Gaiotto and P. Yoo, Gauge theory and the local Geometric Langlands program, to appear.

[44] D. Gaitsgory, Twisted Whittaker model and factorizable sheaves, Selecta Math. 13 (2008) 617, arXiv:0705.4571

[45] D. Gaitsgory, Whittaker categories, http://math.harvard.edu/ gaitsgde/GL/LocWhit.pdf

[46] D. Gaitsgory, Quantum Langlands Correspondence, arXiv:1601.05279

[47] D. Gaitsgory, Master chiral algebra, talk at the Perimeter Institute, March 2018. 
[48] D. Gaitsgory, Parameterization of factorizable line bundles by K-theory and motivic cohomology, arXiv:1804.02567.

[49] D. Gaitsgory and S. Lysenko, Parameters and duality for the metaplectic geometric Langlands theory, arXiv:1608.00284

[50] S.I. Gelfand and Yu.I. Manin, Homological Algebra, Encyclopedia of Mathematical Sciences 38, Springer, 1994.

[51] S. Gukov and E. Witten, Gauge Theory, Ramification, and the Geometric Langlands Program, in Current developments in mathematics, 2006, pp. 35-180, Int. Press, 2008, arXiv:hep-th/0612073

[52] N. Hitchin, The Self-Duality Equations On A Riemann Surface, Proc. London Math. Soc. (3) 55 (1987) 59-126.

[53] V. Kac, S.-S. Roan, and M. Wakimoto, Quantum reduction for affine superalgebras, Comm. Math. Phys. 241 (2003) 307-342.

[54] A. Kapustin, A Note on Quantum Geometric Langlands Duality, Gauge Theory, and Quantization of the Moduli Space of Flat Connections, arXiv:0811.3264.

[55] A. Kapustin and E. Witten, Electric-magnetic Duality And The Geometric Langlands Program, Communications in Number Theory and Physics 1 (2007) 1-236, arXiv:hep-th/0604151

[56] M. Kashiwara and P. Schapira, Sheaves on Manifolds, Springer, 1990.

[57] D. Kazhdan and G. Lusztig, Tensor structures arising from affine Lie algebras, Journal of AMS 6 (1993) 905-947.

[58] M.A.Metlitski, S-duality of $u(1)$ gauge theory with $\theta=\pi$ on non-orientable manifolds: Applications to topological insulators and superconductors, arXiv:1510.05663.

[59] N. Nekrasov and E. Witten, The Omega Deformation, Branes, Integrability, and Liouville Theory, JHEP 1009 (2010) 092, arXiv:1002.0888

[60] A. Polishchuk and M. Rothstein, Fourier transform for D-algebras, Duke Math. J. 109 (2001) 123146.

[61] S. Raskin, Chiral principal series categories II: the factorizable Whittaker category, http://math.mit.edu/ sraskin/cpsii.pdf

[62] S. Raskin, Chiral categories, http://math.mit.edu/ sraskin/chiralcats.pdf

[63] S. Raskin, $\mathcal{W}$-algebras and Whittaker categories, arXiv:1611.04937

[64] G. Segal, The definition of conformal field theory, in Topology, geometry and quantum field theory, LMS Lecture Note Ser. 308, pp. 421-577, Cambridge Univ. Press, Cambridge, 2004.

[65] A. Stoyanovsky, On Quantization of the Geometric Langlands Correspondence I, arXiv:math/9911108

[66] A. Stoyanovsky, A Relation Between the Knizhnik-Zamolodchikov and Belavin-PolyakovZamolodchikov Systems of Partial Differential Equations, arXiv:math-ph/0012013v3.

[67] J. Teschner, Quantization of the Hitchin moduli spaces, Liouville theory, and the geometric Langlands correspondence I, Adv. Theor. Math. Phys. 15 (2011) 471-564, arXiv:1005.2846.

[68] E. Witten, Geometric Langlands and the equations of Nahm and Bogomolny, Proc. Royal Society of Edinburgh, Sec. A: Math. 140 (2010) 857-895, arXiv:0905.4795.

[69] P. Yoo, talk at the Perimeter Institute, October 2016.

Department of Mathematics, University of California, Berkeley, CA 94720, USA

Perimeter Institute for Theoretical Physics, Waterloo, Ontario, N2L 2Y5, Canada 